Aus der Abteilung Kardiologie und Pneumologie

(PD Dr. med. T. Raupach)

im Zentrum Innere Medizin

der Medizinischen Fakultät der Georg-August-Universität Göttingen

\title{
Eine Studie zur Ausbildungssituation deutscher Medizinstudenten hinsichtlich Tabak- und Alkoholabhängigkeit
}

\author{
INAUGURAL - DISSERTATION
}

zur Erlangung des Doktorgrades

der Medizinischen Fakultät

der Georg-August-Universität zu Göttingen

vorgelegt von

Lisa Kathrin Inge Strobel, geb. Müllner

aus

Königstein im Taunus

Göttingen 2012 
Dekan: Prof. Dr. med. H. Kroemer

I. Berichterstatter: Priv.-Doz. Dr. med. T. Raupach, MME

II. Berichterstatter: Prof. Dr. rer. nat. Havemann-Reinecke

III. Berichterstatter: Prof. Dr. med. Hummers-Pradier

Tag der mündlichen Prüfung: 22.01.2013 


\section{Inhaltsverzeichnis}

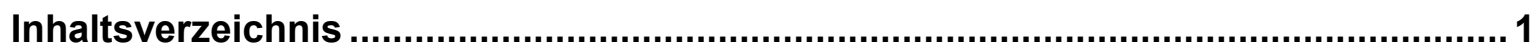

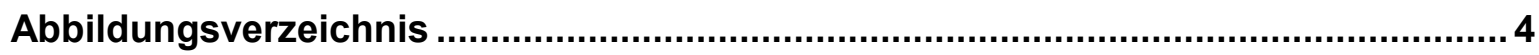

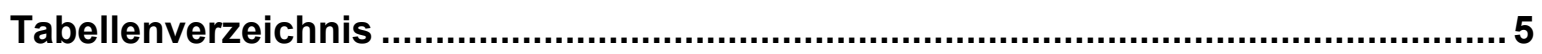

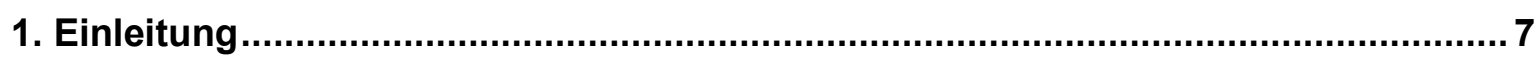

1.1 Aktuelle Todesursachen.......................................................................... 7

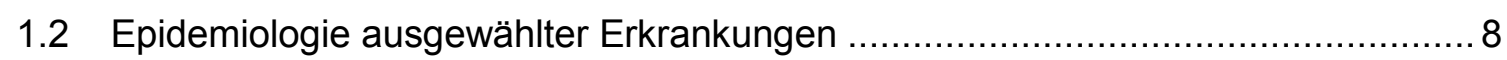

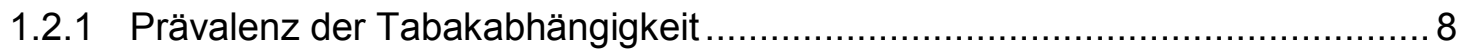

1.2.2 Folgeschäden und gesundheitspolitische Bedeutung der Tabakabhängigkeit . 8

1.2.3 Prävalenz der Alkoholabhängigkeit .................................................... 9

1.2.4 Folgeschäden und gesundheitspolitische Bedeutung der Alkoholabhängigkeit 10

1.2.5 Prävalenz des Diabetes mellitus ......................................................... 11

1.2.6 Folgeschäden und gesundheitspolitische Bedeutung des Diabetes mellitus . 12

1.2.7 Prävalenz der arteriellen Hypertonie ....................................................... 13

1.2.8 Folgeschäden und gesundheitspolitische Bedeutung der arteriellen Hypertonie 13

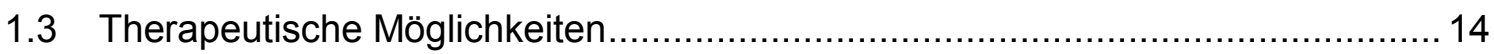

1.1.1. Versorgungsqualität der Tabakabhängigkeit in Deutschland ........................ 18

1.3.1 Versorgungsqualität der Alkoholabhängigkeit in Deutschland ...................... 18

1.3.2 Versorgungsqualität des Diabetes mellitus in Deutschland ......................... 19

1.3.3 Versorgungsqualität der arteriellen Hypertonie in Deutschland .................... 19

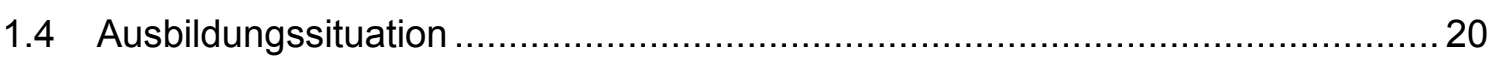

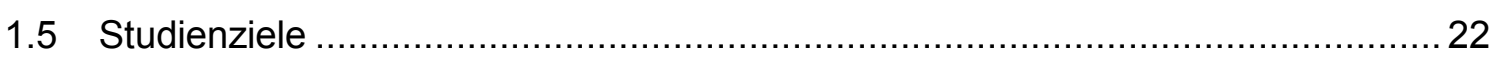

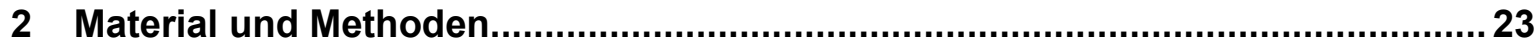

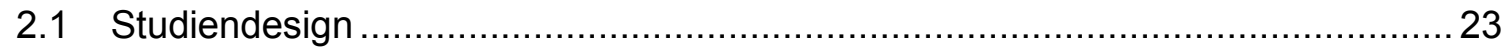

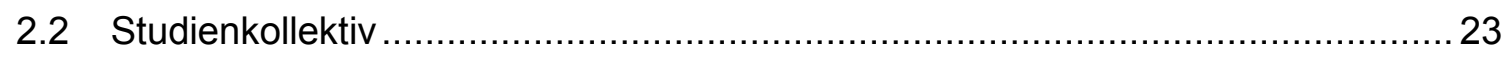

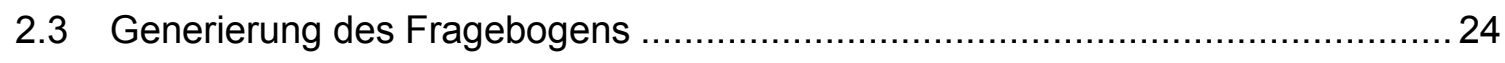

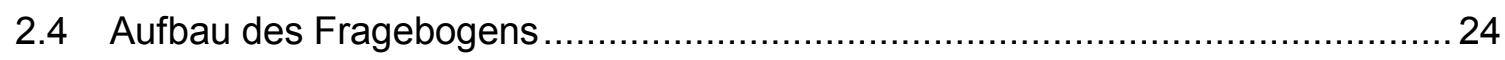

2.4.1 Demographie und Rauchverhalten ..................................................... 24

2.4.2 Komorbidität und Behandlung ausgewählter Erkrankungen ........................ 25

2.4.3 Entwöhnungsmaßnahmen und Beratungsstrategien .................................. 25

Im Zentrum des zweiten Fragebogenteils stand die Untersuchung theoretischer Kenntnisse der Studierenden. 


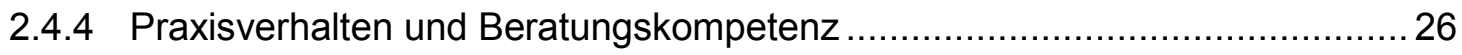

2.4.5 Lehrsituation und Verbesserungswünsche ............................................ 27

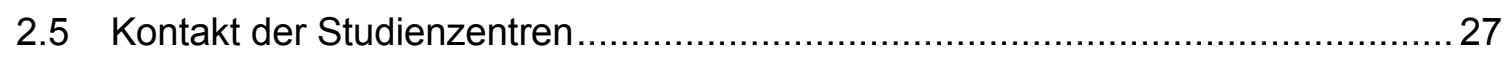

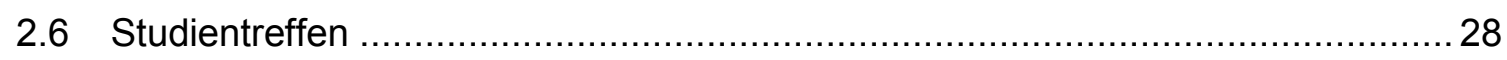

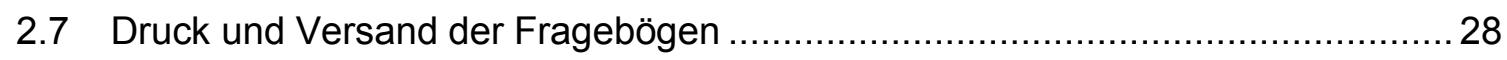

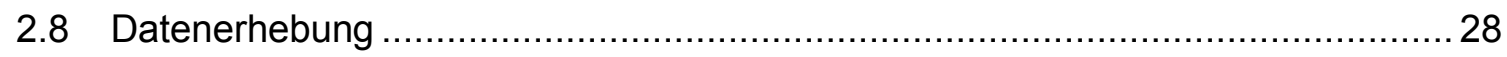

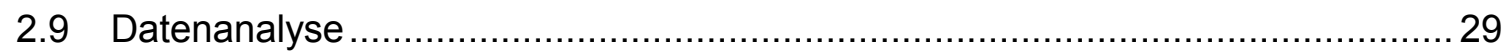

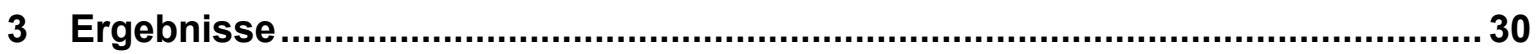

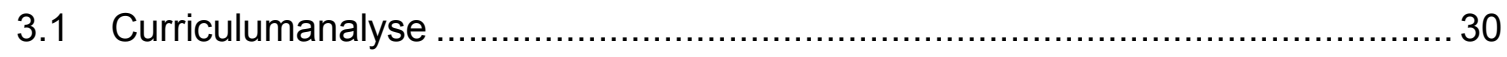

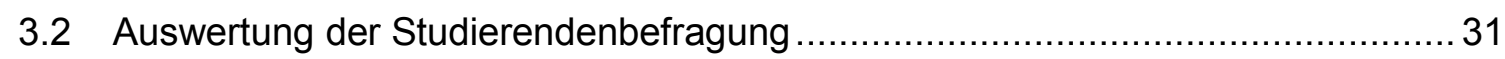

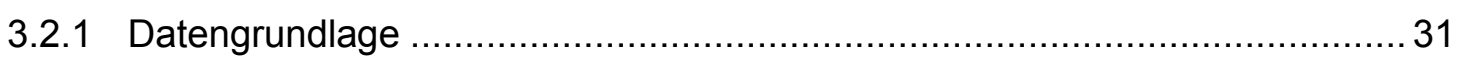

3.2.2 Demographie und Rauchverhalten ...................................................... 31

3.2.3 Komorbidität und Behandlung ausgewählter Erkrankungen ........................ 35

3.2.4 Entwöhnungsmaßnahmen und Beratungsstrategien ................................. 36

3.2.5 Praxisverhalten und Beratungskompetenz ......................................... 41

3.2.6 Lehrsituation und Verbesserungswünsche ............................................. 43

3.3 Unterschiede zwischen Rauchern und Nichtrauchern ..................................... 44

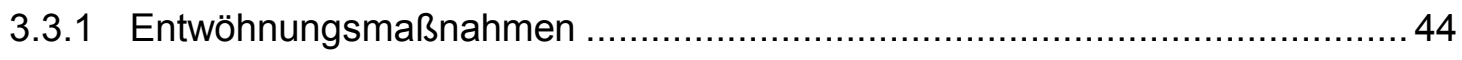

3.3.2 Komorbidität und Behandlung ausgewählter Erkrankungen ...................... 44

3.3.3 Praxisverhalten und Beratungskompetenz ........................................ 44

3.4 Geschlechtsspezifische Unterschiede ................................................... 46

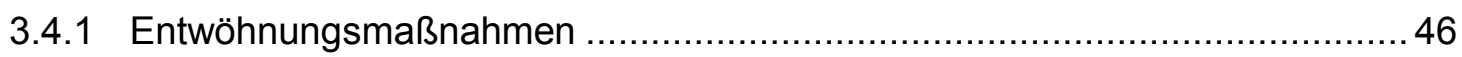

3.4.2 Komorbidität und Behandlung ausgewählter Erkrankungen ....................... 47

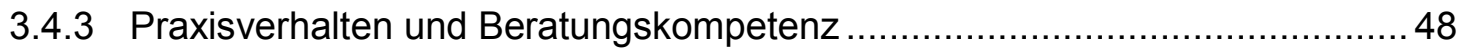

3.5 Unterschiede zwischen Regel- und Modellstudiengang ................................ 48

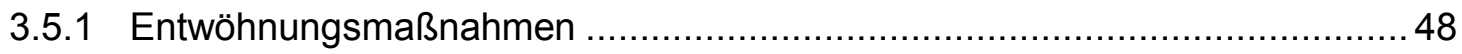

3.5.2 Komorbidität und Behandlung ausgewählter Erkrankungen ....................... 49

3.5.3 Praxisverhalten und Beratungskompetenz ........................................... 50

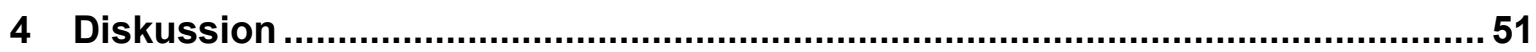

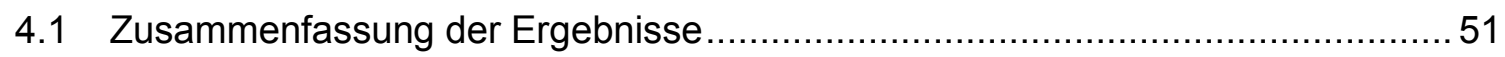

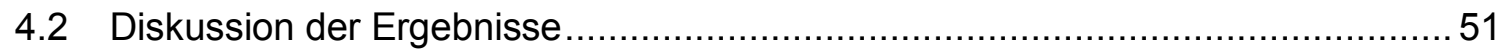

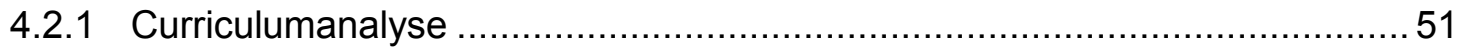

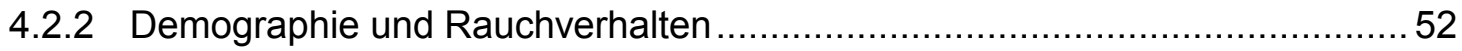

4.2.3 Entwöhnungsmaßnahmen und Beratungsstrategien ................................. 53

4.2.4 Komorbidität und Behandlung ausgewählter Erkrankungen .......................5 53

4.2.5 Praxisverhalten und Beratungskompetenz .......................................... 54

4.2.6 Lehrsituation und Verbesserungswünsche ........................................ 55 


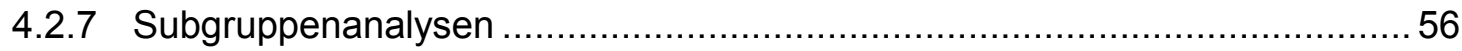

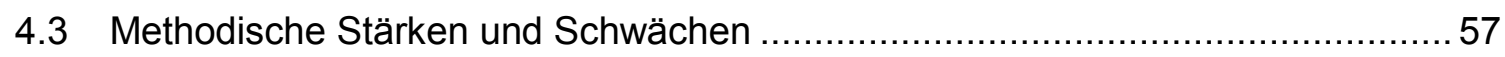

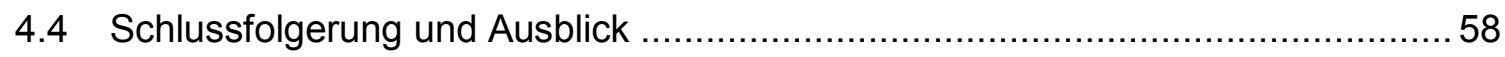

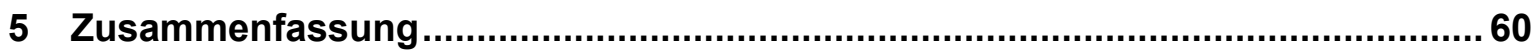

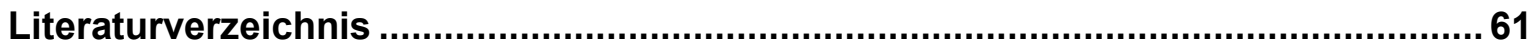

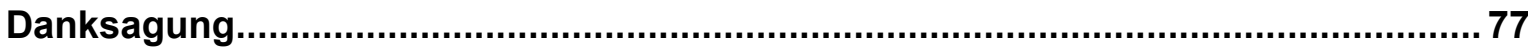

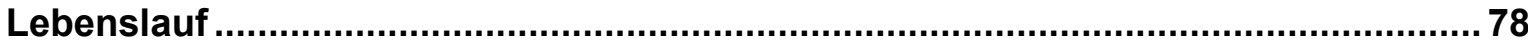




\section{Abbildungsverzeichnis}

Abbildung 1. Studentische Selbsteinschätzung hinsichtlich der Komplikationen und Behandlungsoptionen von Alkohol- und Tabakabhängigkeit, sowie arterieller Hypertonie und Diabetes mellitus

Abbildung 2. Auszug aus dem für die vorliegende Arbeit genutzten Fragebogen zur Effektivität einzelner Tabakentwöhnungsmaßnahmen

Abbildung 3. Auszug aus dem für die vorliegende Arbeit genutzten Fragebogen zur Effektivität einzelner Alkoholentwöhnungsmaßnahmen ............................................. 26

Abbildung 4. Teilnahmebereitschaft der Fakultäten ............................................... 27

Abbildung 5. Studentische Selbsteinschätzung hinsichtlich der Kenntnis zu

Folgeerkrankungen (A) und Behandlungsoptionen (B) von arterieller Hypertonie, Diabetes mellitus, Tabak- und Alkoholabhängigkeit. 38

Abbildung 6. Studentische Einschätzung der Effektivität verschiedener Tabakentwöhnungsmaßnahmen in Abhängigkeit vom Studienjahr. 39

Abbildung 7. Studentische Einschätzung der Effektivität verschiedener Alkoholentwöhnungsmaßnahmen in Abhängigkeit vom Studienjahr. 40

Abbildung 8. Studentische Selbsteinschätzung hinsichtlich der Kompetenz in der Raucherberatung.

Abbildung 9. Anteil der Studenten, die sich eine intensivere Lehre für arterielle

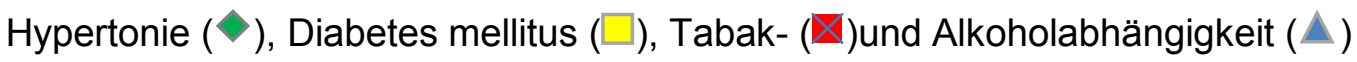
wünschten. 


\section{Tabellenverzeichnis}

Tabelle 1. Komplikationen und Folgeerkrankungen der Tabakabhängigkeit (Auswahl)..... 9

Tabelle 2. Komplikationen und Folgeerkrankungen der Alkoholabhängigkeit (Auswahl) . 11

Tabelle 3. Komplikationen und Folgeerkrankungen des Diabetes mellitus (Auswahl) ..... 13

Tabelle 4. Komplikationen und Folgeerkrankungen der arteriellen Hypertonie (Auswahl) 14

Tabelle 5. Das „5A-Schema“ nach Fiore 2000 zur Behandlung der Tabakabhängigkeit.. 15

Tabelle 6. Langfristige Abstinenzquoten (nach einem Jahr) verschiedener

Tabakentwöhnungsmaßnahmen ..................................................................... 16

Tabelle 7. Bedeutung des Akronyms „FRAMES“ nach Miller WR und Sanchez 1994 ..... 16

Tabelle 8. Langfristige Abstinenzquoten (nach einem Jahr) verschiedener

Alkoholentwöhnungsmaßnahmen

Tabelle 9. Curriculare Verankerung der Lernziele Diabetes mellitus, arterielle Hypertonie,

Alkohol- und Tabakabhängigkeit.

Tabelle 10. Rücklauf der einzelnen Studienzentren. 33

Tabelle 11. Demographische Daten und Rauchcharakteristik 34

Tabelle 12. Raucherquote in den einzelnen Studienjahren 34

Tabelle 13. Anteil der Studenten ( $n$ ), die das öffentliche Rauchverbot befürworten. 35

Tabelle 14. Antworten auf die Frage: „Wie viele der 5 üblichen Maßnahmen in der Tabakentwöhnung, die als „5A“ bekannt sind, können Sie explizit benennen?“.....

Tabelle 15. Antworten auf die Frage: „Wie viele der sechs Maßnahmen in der Beratung alkohol-abhängiger Patienten, deren Anfangsbuchstaben in dem Akronym „FRAMES“ enthalten sind, können Sie explizit benennen?“....

Tabelle 16. Anteil der Studenten ( $n$ ) mit regelmäßigem Patientenkontakt, die angaben, a) den Rauchstatus ihrer Patienten zu erfragen ( $\left.n_{\text {gesamt }}=7.635\right)$ und b) einen Rauchstopp zu empfehlen $\left(n_{\text {gesamt }}=7.628\right)$.

Tabelle 17. Anteil der Studenten, die nach eigenen Angaben während des Studiums theoretischen und/oder praktischen Unterricht zur Tabakentwöhnung erhalten haben. ... 43 Tabelle 18. Anteil der Raucher/Nichtraucher im letzten Studienjahr, die die Effektivität der einzelnen Tabak- und Alkoholentwöhnungsmaßnahmen richtig eingeschätzt haben

Tabelle 19. Anteil der Raucher/Nichtraucher im letzten Studienjahr, die angaben, die Folgeerkrankungen und Behandlungsoptionen der einzelnen Erkrankungen zu kennen. 46 Tabelle 20. Anteil der Männer/Frauen im letzten Studienjahr, die die Effektivität der einzelnen Tabak- und Alkoholentwöhnungsmaßnahmen richtig eingeschätzt haben 47

Tabelle 21. Anteil der Männer/Frauen im letzten Studienjahr, die angaben, die Folgeerkrankungen und Behandlungsoptionen der einzelnen Erkrankungen zu kennen. 48 
Tabelle 22. Anteil der Studenten aus Regel- (RSM) und Modellstudiengängen (MSM) im letzten Studienjahr, die die Effektivität der einzelnen Tabak- und Alkoholentwöhnungsmaßnahmen richtig eingeschätzt haben.

Tabelle 23. Anteil der Studenten aus Regel- (RSM) und Modellstudiengängen (MSM) im letzten Studienjahr, die angaben, die Folgeerkrankungen und Behandlungsoptionen der einzelnen Erkrankungen zu kennen. 


\section{Einleitung}

\subsection{Aktuelle Todesursachen}

Chronische Erkrankungen zählen heutzutage weltweit zu den Hauptursachen für Morbidität und Mortalität. So sind insgesamt 59\% aller weltweiten Todesfälle auf nicht übertragbare Erkrankungen ${ }^{1}$ zurückzuführen. Diese machen wiederum etwa $46 \%$ der weltweiten Krankheitslast aus (Puska et al. 2003). Im Jahr 2002 begründeten übertragbare Krankheiten $^{2}$ etwa $40 \%$ der weltweiten Krankheitslast und waren für ein Drittel aller Todesfälle verantwortlich. Schätzungen zufolge werden diese Krankheiten im Jahr 2030 nur noch $14 \%$ der globalen Mortalität konstituieren (Mathers und Loncar 2006).

Im Gegensatz dazu gewinnen chronische Erkrankungen vor dem Hintergrund demographischer Alterung zunehmend an Bedeutung und spielen nicht nur individuell, sondern auch gesundheitspolitisch und ökonomisch eine bedeutsame Rolle. In Europa sind nicht übertragbare Krankheiten bereits heute für $86 \%$ der Mortalität und $77 \%$ der Krankheitslast verantwortlich. Damit zählt diese Region zu den am stärksten von der Zunahme chronischer Erkrankungen betroffenen Teilen der Welt (Farrington 2004). Führende Todesursachen sind neben Herz-Kreislauf-Leiden Malignome sowie chronische Atemwegserkrankungen. In Deutschland allein sind $40 \%$ aller Todesfälle durch kardiovaskuläre Erkrankungen und 20\% durch Malignome verursacht (World Health Organization 2006). Aber auch in Schwellenländern wird eine Zunahme der Prävalenz und Inzidenz verschiedener chronischer Erkrankungen (Diabetes mellitus Typ 2, kardiovaskuläre Erkrankungen, chronisch obstruktive Lungenerkrankung) für die nächsten Jahre erwartet (Mathers und Loncar 2006).

Darüber hinaus ist bekannt, dass lediglich eine geringe Anzahl von - zum größten Teil vermeidbaren Risikofaktoren die Entstehung dieser Erkrankungen begünstigt und die weltweite Krankheitslast mitbestimmt. Im Jahre 2001 ließen sich 45\% aller weltweiten Todesfälle durch das Einzel- oder Zusammenspiel von nur 19 separaten Risikofaktoren erklären; in den Industriestaaten standen Rauchen, arterielle Hypertonie, Übergewicht, und Alkoholkonsum an erster Stelle (Lopez et al. 2006, Ezzati et al. 2002). Ähnliche Ergebnisse wurden in der Interheart-Studie präsentiert, die zeigte, dass die überwiegende Mehr-

\footnotetext{
${ }^{1}$ Dazu zählen laut WHO: Neubildungen, Diabetes mellitus, kardiovaskuläre, sowie chronische Atemwegs- und MagenDarmerkrankungen (vgl. Mathers und Loncar 2006)

${ }^{2}$ Dazu zählen laut WHO: Malaria, HIV, Tuberkulose, perinatale Erkrankungen, Atemwegsinfektionen, und Mangelernährung (vgl. Mathers und Loncar 2006)
} 
zahl kardiovaskulärer Ereignisse auf nur wenige, modifizierbare Risikofaktoren (Rauchen, Diabetes, arterielle Hypertonie) zurückzuführen ist (Yusuf et al. 2004).

\subsection{Epidemiologie ausgewählter Erkrankungen}

Im folgenden Kapitel werden die Tabak- / Alkoholabhängigkeit, die arterielle Hypertonie und der Diabetes mellitus unter verschiedenen, epidemiologischen Gesichtspunkten näher beleuchtet. Um deren Bedeutsamkeit im Gesundheitssystem detailliert darzulegen, werden in einem ersten Abschnitt jeweils Angaben zur weltweiten Prävalenz und Häufigkeit in Deutschland gemacht, während in einem zweiten Abschnitt die mit der jeweiligen Erkrankung assoziierten Komplikationen / Organschäden, Mortalitätsraten und die daraus resultierende Krankheitslast in Deutschland dargestellt wird.

\subsubsection{Prävalenz der Tabakabhängigkeit}

Schätzungen der WHO zufolge gibt es ca. 1,3 Milliarden Raucher weltweit, was in etwa einem Drittel der Weltbevölkerung entspricht, die älter als 15 Jahre ist (Shafey et al 2003). Nahezu eine Milliarde der Raucher sind Männer und ca. 84\% aller Raucher leben in Entwicklungsländern (Esson und Leeder 2004). Bereits in der Altersgruppe der 13- bis 15Jährigen konsumiert nahezu ein Fünftel Tabakprodukte, wie Ergebnisse aus dem Global Youth Tobacco Survey im Jahr 2006 belegten (Centers for Disease Control and Prevention 2006). Im Falle fehlender gesundheitspolitischer Interventionen ist zu erwarten, dass die Anzahl der Raucher weiter ansteigen wird (Guindon und Boisclair 2003).

In Deutschland bezeichneten sich im Jahr 2009 insgesamt 30,5\% der Männer und 21,2\% der Frauen als gelegentliche oder regelmäßige Raucher (Statistisches Bundesamt Wiesbaden 2011). Eine aktuelle Erhebung an 6813 Jugendlichen im Alter von 11 bis 17 Jahren ergab bereits in dieser Altersgruppe eine Raucherprävalenz von 20,4\%, wobei die Zahlen für beide Geschlechter ähnlich hoch waren (Müller - Riemenschneider et al. 2010).

\subsubsection{Folgeschäden und gesundheitspolitische Bedeutung der Tabakabhängigkeit}

Sowohl Rauchen als auch die Exposition gegenüber Passivrauch begünstigen eine Vielzahl von Erkrankungen (siehe Tabelle 1). Dadurch haben Raucher eine im Durchschnitt 10 Jahre geringere Lebenserwartung als Nichtraucher und eine schlechtere Lebensqualität (Clarke et al. 2009, Strandberg et al. 2008). Etwa zwei Drittel aller Raucher sterben an diesen rauchbedingten Erkrankungen (Doll et al. 2004). In Deutschland waren im Jahr 2003 13,4\% aller Todesfälle (114.467) rauchbedingt (Neubauer et al. 2006). Ohne die Umsetzung entsprechender Maßnahmen zur Tabakprävention beziehungsweise zur 
nachhaltigen Reduzierung des Tabakkonsums ist davon auszugehen, dass die Anzahl der tabakbedingten Todesfälle weiter ansteigen wird (Mathers und Loncar 2006).

Mit den zahlreichen durch das Rauchen verursachten Erkrankungen und Todesfällen sind auch immense finanzielle Belastungen für das deutsche Gesundheitssystem verknüpft. Neubauer et al. schätzten die Gesamtkosten im Jahr 2006 auf etwa 21 Milliarden Euro, wobei direkte Kosten (Behandlung rauchassoziierter Erkrankungen) etwa ein Drittel der Gesamtmenge ausmachten und zur Hälfte durch kardiovaskuläre Erkrankungen verursacht worden sind. Die Prävention dieser Erkrankungen durch eine Senkung der Raucherquote und eine Verhinderung der Suchtentwicklung bei Jugendlichen muss daher eine prioritäre Rolle im Gesundheitssystem spielen.

\begin{tabular}{ll} 
Komplikationen \& Folgeerkrankungen & Referenz \\
\hline Koronare Herzkrankheit \& Herzinfarkt & Teo et al. 2006 \\
Schlaganfall & Wolf et al. 1991, Rodriguez et al. 2002 \\
Periphere arterielle Verschlusskrankheit & Willigendael et al. 2004, Alzamora et al. 2010 \\
COPD / untere Atemwegserkrankungen & Doll et al. 2004, Lokke et al. 2006 \\
$\begin{array}{l}\text { Rauchinduzierte Neoplasien } \\
\text { (Mundhöhle, Larynx, Pharynx, Lunge, Ösophagus, }\end{array}$ & Centers for Disease Control and Prevention 200 \\
$\begin{array}{l}\text { Magen, Pankreas, Niere, Blase, Zervix, akute } \\
\text { myeloische Leukämie) }\end{array}$ & \\
Schwangerschaftskomplikationen / Plötzlicher Kindstod & Rogers 2009, Moon et al. 2007 \\
\hline
\end{tabular}

Tabelle 1. Komplikationen und Folgeerkrankungen der Tabakabhängigkeit (Auswahl)

\subsubsection{Prävalenz der Alkoholabhängigkeit}

Nach Anderson konsumieren knapp zwei Milliarden Menschen weltweit regelmäßig Alkohol (Anderson 2006). Der durchschnittliche Pro-Kopf-Verbrauch reinen Alkohols (definiert als $100 \%$ Ethanol) variiert dabei länderabhängig sehr stark und beträgt im globalen Mittel etwa 6,2 Liter pro Jahr (Rehm et al. 2009). Deutschland zählt weltweit zu den Ländern mit dem höchsten Alkoholkonsum (Coder et al. 2009). Im Jahr 2008 betrug der Pro-KopfVerbrauch reinen Alkohols in Deutschland 8,8 Liter (Gärtner et al. 2010).

Zur Prävalenz alkoholbezogener Störungen existieren nur wenige epidemiologische Studien in Deutschland. Hochrechnungen auf Grundlage der Lübecker TACOS ${ }^{3}$-Studie und der Bundesstudie 1997 (Kraus und Bauernfeind 1998) ergaben für die Altersgruppe der 18- bis 59-Jährigen eine nach DSM-IV ermittelte 12-Monatsprävalenz von 2,4\% für Alko-

\footnotetext{
${ }^{3}$ TACOS $=$ Transitions in Alcohol Consumption and Smoking
} 
holabhängigkeit (1,6 Millionen Personen) und ca. 4\% für Alkoholmissbrauch (2,65 Millionen Personen). Die Diagnose einer Alkoholabhängigkeit fordert gemäß DSM-IV das gleichzeitige Vorliegen von mindestens drei der nachfolgenden Kriterien innerhalb der letzten 12 Monate (starker Wunsch Alkohol zu konsumieren, verminderte Kontrollfähigkeit über den Konsum, körperliche Entzugserscheinung bei Konsumstopp, Nachweis einer Toleranz, Vernachlässigung anderer Interessen zugunsten des Substanzkonsums, fortschreitender Konsum trotz Nachweis schädlicher Folgen). Ein Alkoholmissbrauch nach DSM-IV liegt vor, wenn die/der Betroffene noch nicht die Kriterien eines Abhängigkeitssyndroms erfüllt, jedoch bereits konsumbedingte physische und/oder psychische Schäden entstanden sind (Kraus et al. 2008). Im Rahmen des epidemiologischen Suchtsurveys 2006 erfüllten insgesamt 2,7\% der Teilnehmer die DSM-IV Kriterien für Alkoholabhängigkeit und 5,1\% die des Alkoholmissbrauches (Kraus et al. 2008). Die höchste Missbrauchsrate lag in der Gruppe der 18- bis 24-Jährigen (Kraus et al. 2008).

\subsubsection{Folgeschäden und gesundheitspolitische Bedeutung der Alkoholabhängig- keit}

Akuter und chronischer Alkoholkonsum können zu lebensbedrohlichen Erkrankungen (siehe Tabelle 2) und Verlust an Lebenszeit führen. Für alkoholabhängige Personen verkürzt sich die Lebenserwartung um durchschnittlich 23 Jahre (Statistisches Bundesamt Wiesbaden 1999).

Der Anteil alkoholbedingter Todesfälle an allen Todesfällen in Deutschland, die sich im Alter zwischen 35 und 64 Jahren ereignen, betrug bei Männern 25\% und bei Frauen 13\% (John und Hanke 2002). Insgesamt traten $75 \%$ aller alkoholbedingten Todesfälle vor dem 65. Lebensjahr auf (John und Hanke 2002). Im Jahr 2003 wurde darüber hinaus nachgewiesen, dass ca. $10 \%$ aller stationären Aufenthalte in deutschen Krankenhäusern auf die schädliche Wirkung von Tabak und/oder Alkohol zurückzuführen sind (Hanke und John 2003). Die Liegedauer war zudem gegenüber nicht Tabak und/oder Alkohol bezogenen Fällen um 1,5 Tage erhöht. Schätzungen zufolge beläuft sich der jährliche volkswirtschaftliche Schaden auf circa 20 Milliarden $€$ (ca. 1\% des Bruttoinlandproduktes im Jahr 2002 (Konnopka und König 2007)). Diese finanziellen Verluste entstehen zum einen direkt durch Ressourcenverbrauch (Behandlung alkoholassoziierter Erkrankungen) und zum anderen indirekt durch Ressourcenverlust wie hohe Arbeitsunfähigkeits- und Frührentenquoten (Bergmann und Horch 2002, Konnopka und König 2007). Gesundheitspolitisch kommt deswegen der Prävention und der Alkoholentwöhnung eine vordringliche Aufgabe im Gesundheitssystem zu. 


\begin{tabular}{ll} 
Komplikationen \& Folgeerkrankungen & Referenz \\
\hline Dilatative Kardiomyopathie & Fatjó et al. 2007 \\
Herzrhythmusstörungen & Uyarel et al. 2005 \\
Arterielle Hypertonie & Puddey \& Beilin 2006 \\
Koronare Herzkrankheit & Britton \& Marmot 2004 \\
Schlaganfall & Britton \& Marmot 2004, Klatsky 2009 \\
Wernicke - Korsakoff-Syndrom & Thomson \& Marshall 2006, Pitel et al. 2007 \\
Cerebrale Atrophie & Oscar-Berman \& Marinkovic 2007 \\
Alkoholische Polyneuropathie & Vittadini et al. 2001 \\
Alkoholische Fettleber / Leberzirrhose & Szucs et al. 2005 \\
akute/chronische Pankreatitis & Ramstedt 2004 \\
Gastritis / gastrointestinale Blutung & Teyssen \& Singer 2003 \\
Alkoholinduzierte Neoplasien & Thun et al. 1997, Corrao et al. 2004, Polednak 2005 \\
(Mundhöhle, Pharynx, Larynx, Ösophagus, & \\
Leber, Pankreas, Mamma, Kolon/Rektum) & \\
Alkoholembryopathie & Alati et al. 2006 \\
\hline
\end{tabular}

Tabelle 2. Komplikationen und Folgeerkrankungen der Alkoholabhängigkeit (Auswahl)

\subsubsection{Prävalenz des Diabetes mellitus}

Weltweit ist eine Zunahme der Prävalenz des Diabetes mellitus zu beobachten. Während im Jahre 2000 ca. 171 Millionen Menschen an Diabetes erkrankt waren, wird für das Jahr 2030 eine Verdopplung der Erkrankungszahlen (366 Millionen) vorausgesagt (Wild et al. 2003). Schätzungen der International Federation of Diabetes (IDF) aus dem Jahre 2009 zufolge leiden europaweit 55 Millionen Menschen an Diabetes mellitus, was einer Prävalenz in der erwachsenen Bevölkerung (Alter: 20-79 Jahre) von 8,5\% entspricht.

Laut selbstanamnestischen Angaben im Bundesgesundheitssurvey 1997/1998 waren 4,7\% der deutschen Männer und 5,6\% der deutschen Frauen im Alter zwischen 18 und 79 Jahren von einem Diabetes mellitus betroffen (Thefeld 1999). Hauner geht im Jahr 2005 von ca. 6 Millionen bekannten Diabetikern in Deutschland aus. Die Dunkelziffer, d.h. die Anzahl der nicht diagnostizierten Diabetiker, ist aber vermutlich um ein Vielfaches größer und liegt in dem initial häufig symptomarmen Krankheitsverlauf begründet. Untersuchungen aus dem Raum Augsburg zeigten in der Altersgruppe 55 bis 74 Jahre eine Prävalenz des unentdeckten Diabetes mellitus von $8,2 \%$, die damit etwa genauso groß war wie die Häufigkeit des bekannten Diabetes (8,4\%) in dieser Altersgruppe (Rathmann et al. 2009). Die Prävalenz des Diabetes mellitus nimmt im Alter stetig zu. Ab dem 60. Lebensjahr beträgt sie zwischen 15 und 25\% (Hauner 2005), und nahezu jede fünfte deutsche Frau von 70 bis 79 Jahren ist an einem Diabetes mellitus erkrankt. 


\subsubsection{Folgeschäden und gesundheitspolitische Bedeutung des Diabetes mellitus}

Als Folge des Diabetes können zahlreiche und verschiedenartige Komplikationen auftreten (siehe Tabelle 3). Neben Diabetes-spezifischen Mikroangiopathien (Retinopathie, Nephropathie, Neuropathie), spielen makrovaskuläre Komplikationen (koronare Herzkrankheit, periphere arterielle Verschlusskrankheit, Schlaganfall) vor allem bei Typ-2Diabetikern immer häufiger eine entscheidende Rolle. Insgesamt ist die Lebenszeiterwartung deutlich verkürzt; nach 30jähriger Beobachtung aller neuentdeckten Diabetiker in Mecklenburg-Vorpommern wurde ein mittlerer Lebenszeitverlust von 5,3 Jahren bei männlichen und 6,4 Jahren bei weiblichen Patienten registriert (Schneider et al. 1993).

In der Diabetes-Interventions-Studie (DIS 1996) konnte gezeigt werden, dass das relative Gesamtmortalitätsrisiko für 36- bis 45-jährige Männer mit Typ-2-Diabetes im Verhältnis zur Normalbevölkerung fünffach und bei 36- bis 45-jährigen Frauen siebenfach erhöht war (Hanefeld et al. 1996). Im Jahr 2010 schätzten Roglic und Unwin mithilfe eines computerisierten Krankheitslastmodells die Diabetes-bedingte Mortalität in Europa auf circa $11 \%$. Untersuchungen zur Anzahl Diabetes-bedingter Todesfälle in Deutschland wurden vom Statistischen Bundesamt vorgelegt. Hiernach waren im Jahr 2009 knapp 3\% aller Todesfälle auf Diabetes mellitus zurückzuführen (Statistisches Bundesamt Deutschland 2011). Die Abhängigkeit der Todesursachenstatistik von der Qualität der ärztlichen Feststellungen auf den Todesbescheinigungen ist bei der Interpretation der Ergebnisse jedoch zu berücksichtigen (Fuller et al. 1983).

Auf der Basis von deutschen Krankenkassendaten wurden im Rahmen der KoDim ${ }^{4}$ - Studie diabetesbedingte Kosten von 22,3 Milliarden Euro pro Jahr ermittelt. Während 14,6 Milliarden Euro durch direkte Kosten (Krankenversorgung, Pflegeversicherung) entstanden, sind 7,7 Milliarden Euro auf indirekte Kosten (Arbeitsunfähigkeit, Frühberentung) zurückzuführen. Lediglich ein Fünftel der Ausgaben entfiel auf die Behandlung der eigentlichen Grunderkrankung (stationärer Aufenthalt zur Blutzuckereinstellung, Kosten für Antidiabetika, Blutzuckermessungen), wohingegen knappe vier Fünftel durch Behandlung und Pflege der diabetischen Begleit- oder Folgeerkrankungen verursacht wurden (Köster et al. 2006). Aufgrund von steigenden Erkrankungszahlen wird Deutschland Versorgungsengpässen und Kostenanstiegen im Gesundheitssystem gegenüberstehen. Eine Umsetzung primär und sekundär präventiver Maßnahmen ist daher von dringlichster Notwendigkeit.

\footnotetext{
${ }^{4}$ KoDim $=$ Kosten des Diabetes mellitus
} 


\begin{tabular}{ll} 
Komplikationen \& Folgeerkrankungen & Referenz \\
\hline Koronare Herzkrankheit \& Herzinfarkt & Löwel et al. 1999 \\
Schlaganfall & Rodriguez et al. 2002 \\
Periphere arterielle Verschlusskrankheit & Beks et al. 1995, Aron son 2008 \\
Diabetische Polyneuropathie & Vinik et al. 2003, Aring et al. 2005 \\
Diabetische Retinopathie / Makulopathie & Icks et al. 1997 \\
Diabetische Nephropathie & Brancati et al. 1997, Adler et al. 2003 \\
Diabetisches Fußsyndrom & Heller 2004 \\
Diabetische Embryo-/ Fetopathie & Buchanan \& Kitzmiller 1994 \\
\hline
\end{tabular}

Tabelle 3. Komplikationen und Folgeerkrankungen des Diabetes mellitus (Auswahl)

\subsubsection{Prävalenz der arteriellen Hypertonie}

Kearney et al. ermittelten für das Jahr 2000 eine weltweite Prävalenz der arteriellen Hypertonie von 26,4\% (972 Millionen Betroffene). Für das Jahr 2025 wurde eine Zunahme der Erkrankungsrate um über 60\% (1,56 Milliarden) prognostiziert.

Im Rahmen des Bundesgesundheitssurveys 1997/1998 konnte in Deutschland eine Häufigkeit der arteriellen Hypertonie von knapp 30\% für Männer und 26,9\% für Frauen konstatiert werden (Thamm 1999). In der Gruppe der 25- bis 64-Jährigen fanden sich, je nach Publikation (KORA ${ }^{5}-2000$ und SHIP ${ }^{6}$ Studie), Werte zwischen 23-32\% für Frauen und 36-57\% für Männer bei steigender Häufigkeit mit zunehmendem Alter (Löwel et al. 2006). Aktuellen Erhebungen zufolge leidet bereits im jungen Lebensalter (11-17 Jährige) jeder Zehnte an Bluthochdruck (Müller-Riemenschneider et al. 2010).

\subsubsection{Folgeschäden und gesundheitspolitische Bedeutung der arteriellen Hyper- tonie}

Die Folgen und Komplikationen des Bluthochdrucks sind vielfältig und wirken sich insbesondere in den Gefäßsystemen von Herz und Gehirn aus (siehe Tabelle 4). Bluthochdruck stellt demnach einen der wesentlichen Risikofaktoren für Herzinfarkt (Yusuf et al. 2004) und den Hauptrisikofaktor für Schlaganfall (Straus et al. 2002) dar. In Deutschland ist der Bluthochdruck für insgesamt ein Viertel der Gesamtmortalität verantwortlich (World Health Organization 2005).

Im Hinblick auf direkte und indirekte Krankheitskosten nimmt er dadurch eine besondere Stellung im deutschen Gesundheitssystem ein.

\footnotetext{
${ }^{5}$ KORA = Kooperative Gesundheitsforschung in der Region Augsburg

${ }^{6}$ SHIP = Study of Health in Pomerania
} 
Die direkten Kosten für Krankheiten des Herzkreislaufsystems betrugen im Jahr 2006 ca. 35,2 Milliarden $€$, wovon rund 8,6 Milliarden $€$ auf die arterielle Hypertonie entfielen (Jahnsen et al. 2008). Allein 7,2 Milliarden $€$ wurden durch Kosten aus dem ambulanten Versorgungsbereich - zu drei Viertel Apothekenleistungen - verursacht. Durch Arbeitsausfall, Invalidität oder vorzeitigen Tod ist der Bluthochdruck weiterhin für insgesamt 27.000 verlorene Erwerbstätigkeitsjahre verantwortlich. Das Durchschnittsalter bei Rentenbeginn infolge von Hypertonie lag im Jahr 2006 bei beiden Geschlechtern bei 55 Jahren.

\begin{tabular}{ll} 
Komplikationen \& Folgeerkrankungen & Referenz \\
\hline Koronare Herzkrankheit \& Herzinfarkt & Yusuf et al. 2004 \\
Schlaganfall & Straus et al. 2002 \\
Bauchaortenaneurysma / Aortendissektion & Upchurch \& Schaub 2006, Spittel et al. 1993 \\
Hypertensive Nephropathie & Obialo et al. 2002 \\
Hypertensive Retinopathie & Schubert 1998, Wong \& Mitchell 2007 \\
\hline
\end{tabular}

Tabelle 4. Komplikationen und Folgeerkrankungen der arteriellen Hypertonie (Auswahl)

\subsection{Therapeutische Möglichkeiten}

Im Folgenden sollen die therapeutischen Ansätze zur Behandlung aller vier Erkrankungen kurz skizziert werden.

Mittlerweile konnte gezeigt werden, dass die Tabakabhängigkeit durch Kurzinterventionen, pharmakologische und/oder verhaltenstherapeutische Behandlung effektiv therapiert werden kann (Aveyard und West 2007, Lancaster et al. 2000, Cahill et al. 2008, Fiore et al. 2008).

Ohne professionelle Unterstützung gelingt es jedoch nur 2-5 \% aller Raucher, das Rauchen dauerhaft einzustellen (West et al. 2000), obwohl etwa zwei Drittel aller Raucher den Tabakkonsum gerne aufgeben möchten (Hughes 1999).

Da etwa $70 \%$ aller Raucher mindestens einmal im Jahr einen Arzt aufsuchen (Chatkin J und Chatkin G 2009) und sich bereits eine kurze hausärztliche Intervention signifikant auf die langfristigen Abstinenzquoten auswirkt (Stead et al. 2008a), ergibt sich hieraus eine ideale Interventionsmöglichkeit. Als Grundsäulen der Kurzintervention gelten die erstmals im Jahr 2000 von Fiore beschrieben „5 A“. Sie dienen als Orientierungshilfe, Rauchgewohnheiten der Patienten zu erfragen, zu dokumentieren und die Aufhörmotivation zu erörtern (siehe Tabelle 5). 


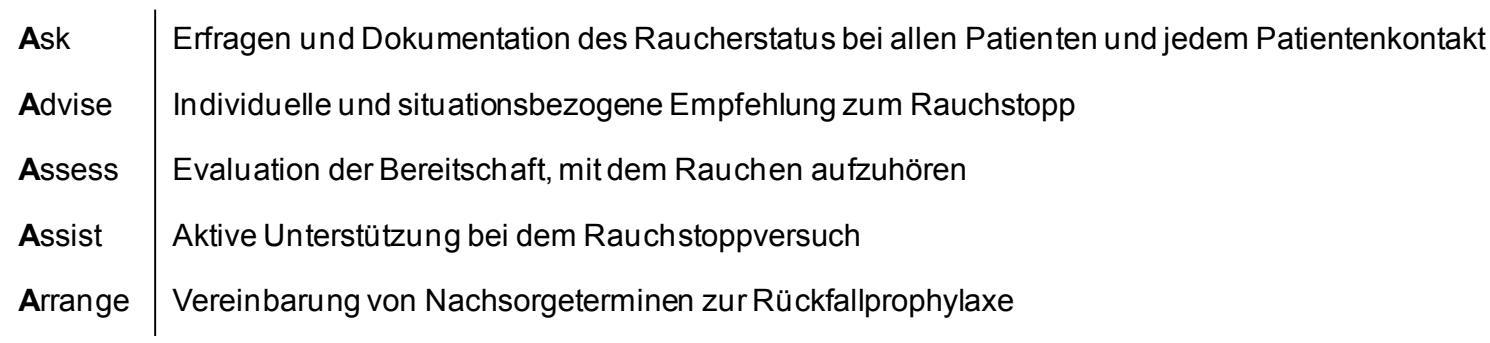

Tabelle 5. Das „5 A-Schema“ nach Fiore 2000 zur Behandlung der Tabakabhängigkeit

Zur medikamentösen Therapie zählen neben Nikotin-Ersatz-Präparaten die Wirkstoffe Bupropion und Vareniclin, deren Wirksamkeit in großen Metaanalysen nachgewiesen wurde (Stead et al. 2008b, Hughes et al. 2007, Cahill et al. 2008).

Nikotin-Ersatz-Präparate existieren in verschiedenen Darreichungsformen (Nikotinpflaster, Nikotinkaugummi, Nikotintabletten, Nikotinnasenspray, Nikotininhaler) und erzielen durch eine kontinuierliche Abgabe von Nikotin eine Verminderung der Entzugssymptomatik.

Der ursprünglich als Antidepressivum zugelassene Wirkstoff Bupropion wird seit dem Jahr 1997 auch erfolgreich in der Tabakentwöhnung eingesetzt. Obgleich sein genauer Wirkmechanismus noch unklar ist (Paterson 2009), führt eine regelmäßige Einnahme von Bupropion zu einer signifikanten Reduktion des Rauchverlangens.

Ein noch recht neuer Wirkstoff in der Therapie der Tabakabhängigkeit ist das seit 2007 in Deutschland verfügbare Vareniclin. Durch Bindung an nikotinerge AcetylcholinRezeptoren des Subtyps $\alpha_{4} \beta_{2}$ wird die Nikotinwirkung imitiert und Entzugssymptome gemildert. Gleichzeitig wird der Rezeptor dabei für extern zugeführtes Nikotin blockiert.

Alternative Entwöhnungsmaßnahmen wie Selbsthilfeliteratur und Akupunktur sind wenig bis gar nicht effektiv (Lancaster und Stead 2005, White et al. 2011). Die bislang höchsten Abstinenzquoten erzielen kombinierte Entwöhnungsprogramme, bei denen Gruppentherapien medikamentös unterstützt werden (Fiore et al. 2008). Die Effektivitäten der aufgeführten Entwöhnungsmaßnahmen, definiert durch Abstinenzquoten nach einem Jahr, sind in Tabelle 6 zusammengefasst. 


\begin{tabular}{lll} 
Entwöhnungsmaßnahme & Abstinenzquote & Referenz \\
\hline Willenskraft allein & $2-5 \%$ & West et al. 2000, Hughes et al. 2004 \\
Hausärztliche Beratung & $\sim 10 \%$ & Stead et al. 2008a \\
Nikotin-Ersatz-Therapie & $\sim 10 \%$ & Stead et al. 2008b \\
Antidepressiva & $\sim 15 \%$ & Hughes et al. 2007 \\
Partielle nAchR-Agonisten & $15-20 \%$ & Cahill et al. 2008 \\
Selbsthilfeliteratur & $2-6 \%$ & Lancaster \& Stead 2005 \\
Akupunktur & $2-3 \%$ & White et al. 2011 \\
Kombiniertes Entwöhnungsprogramm & $\sim 30 \%$ & Fiore et al. 2008 \\
\hline
\end{tabular}

Tabelle 6. Langfristige Abstinenzquoten (nach einem Jahr) verschiedener Tabakentwöhnungsmaßnahmen

Auch die Alkoholabhängigkeit lässt sich effektiv durch Kurzinterventionen, pharmakologische und/oder verhaltenstherapeutische Behandlung therapieren (Miller WR und Wilbourne 2002, Kaner et al. 2009, Fleming 2004/2005, Anton et al. 2006, Brewer 2005). Kurzinterventionen sind kosteneffektiv und können - mit Ausnahme der schweren Alkoholabhängigkeit, die einer intensivierten, fachärztlichen Betreuung bedarf (Krampe et al. 2007, Schuckit 2009) - leicht von Ärzten und nichtärztlichem Personal durchgeführt werden (Babor et al. 2006). Wesentliche Elemente erfolgreicher Kurzintervention sind analog zu den 5 A in dem Akronym „FRAMES“ enthalten, das im Jahr 1994 von Miller und Sanchez beschrieben wurde (siehe Tabelle 7).

\begin{tabular}{l|l} 
Feedback & Rückmeldung des Alkoholkonsums \\
Responsability & Bewusstmachen der eigenen Verantwortlichkeit \\
Advice & Empfehlung einer Alkoholentwöhnung \\
Menue of options & Darstellung von Behandlungsoptionen \\
Empathy & Empathische Gesprächsführung \\
Self-Efficacy & Bewusstmachen der eigenen Selbstwirksamkeit
\end{tabular}

Tabelle 7. Bedeutung des Akronyms „FRAMES“ nach Miller WR und Sanchez 1994

In der medikamentösen Therapie kommen pharmakologische Substanzen wie Disulfiram, Acamprosat und Naltrexon zum Einsatz. In großen Meta-Analysen konnte gezeigt werden, dass die Gabe des Alkoholaversivums Disulfiram - sofern sie unter therapeutischer Supervision erfolgt - sehr effektiv ist (Berglund 2005, Brewer 2005, Krampe und Ehrenreich 2010). Auch Acamprosat und Naltrexon führen nachweislich zur Reduktion von schweren Rückfällen und Trinkhäufigkeit und können im Gegensatz zu Disulfiram auch bei noch bestehendem Trinkverhalten eingenommen werden (Mann et al. 2004, Garbutt 2010, Rösner et al. 2010). Stationär durchgeführte Entgiftungen ohne Anschlussbehand- 
lung führen lediglich bei 15\% aller alkoholabhängigen Patienten zu langfristiger Abstinenz (Bottlender 2007). Besonders hohe Abstinenzquoten erzielen dahingegen qualifizierte Entwöhnungsprogramme, die im Anschluss an die stationäre Behandlung stattfinden. Im Rahmen der OLITA ${ }^{7}$-Studie, in der ein mehrphasiges biopsychosoziales Therapiekonzept zur Anwendung kam, wurden Abstinenzquoten von über $50 \%$ und Wiedereinstellungsquoten von $60 \%$ nach 9 Jahren erreicht (Krampe et al. 2007).

Für die Effektivität von Selbsthilfegruppen als einziges Mittel zur Alkoholentwöhnung finden sich wenig wissenschaftliche Nachweise (Ferri et al. 2006), wohingegen eine therapiebegleitende Teilnahme sehr unterstützend auf den Therapieerfolg wirkt (Moos et al. 2001, Frydrych et al. 2009). Alternative Therapien zur Alkoholentwöhnung wie Akupunktur und Selbsthilfeliteratur zeigen kaum bis keinen Effekt (Lu et al. 2009, Sanchez-Craig et al. 1996). Die Effektivitäten der aufgeführten Entwöhnungsmaßnahmen, definiert durch Abstinenzquoten nach einem Jahr, sind in Tabelle 8 zusammengefasst.

\begin{tabular}{|c|c|c|}
\hline Entwöhnungsmaßnahme & Abstinenzquote & Referenz \\
\hline Willenskraft allein & * & Moyer und Finney 2002 \\
\hline Hausärztliche Beratung & $25 \%$ & $\begin{array}{l}\text { Miller WR \& Wilbourne 2002, Vasilaki et al } \\
2006\end{array}$ \\
\hline Turboentzug & * & Moyer und Finney 2002 \\
\hline Selbsthilfegruppen & $25 \%$ & Moos et al. 1999 \\
\hline Selbsthilfeliteratur & Kein Effekt & Sanchez-Craig et al. 1996 \\
\hline Akupunktur & Kein Effekt & Lu et al. 2009 \\
\hline Stationärer Entzug & $15 \%$ & Bottlender 2007 \\
\hline $\begin{array}{l}\text { Stationärer Entzug \& Entwöhnung } \\
\text { (langfristige ambulante medikamentöse \& } \\
\text { psychotherapeutische Weiterbetreuung) }\end{array}$ & $\sim 75 \%$ & Krampe et al. 2007 \\
\hline
\end{tabular}

Tabelle 8. Langfristige Abstinenzquoten (nach einem Jahr) verschiedener Alkoholentwöhnungsmaßnahmen

* Untersuchung zur Abstinenzrate von nicht behandelten alkoholabhängigen Patienten (Kontrollgruppe, Placebogruppe) in randomisierten klinischen Studien. Eine Metaanalyse von 17 Studien zeigte eine gemittelte Abstinenzrate von 22\%, jedoch betrug der Zeitpunkt des Follow-Ups in $75 \%$ der Studien weniger als ein Jahr. Darüber hinaus wurde keine einheitliche Definition für Abstinenz benutzt.

Primäres Ziel in der Therapie des Diabetes mellitus ist eine euglykämische Stoffwechsellage. Diese ist nach aktuellen Leitlinien der deutschen Diabetes Gesellschaft als ein HbA1c < 7\% (Typ-1-Diabetes) beziehungsweise < 6,5\% (Typ-2-Diabetes) definiert. Während Typ-1-Diabetiker einer lebenslangen exogenen Insulinzufuhr bedürfen, kann der Typ-2-Diabetes anfänglich mit Diät, Bewegungsaktivierung und oralen Antidiabetika therapiert werden. Für weitere Behandlungsempfehlungen sei auf die einschlägigen Leitlinien verwiesen (Martin et al. 2007, Matthaei et al. 2008).

\footnotetext{
${ }^{7}$ OLITA = Outpatient long-term intensive therapy of alcoholics
} 
In der Behandlung der arteriellen Hypertonie bilden Lebensstilveränderungen (Rauchstopp, Gewichtsreduktion, Reduktion des Alkoholkonsums, körperliche Bewegung, gesunde Ernährung, Reduktion des Kochsalzkonsums) eine wichtige Grundlage (Puddey et al. 1997, Whelton et al. 1998, Jennings 1997, Sacks et al. 2001), können die frühzeitige medikamentöse Therapie aber nicht ersetzen. Zur medikamentösen Therapie stehen mehrere Wirkstoffklassen zur Verfügung, die in Mono- oder Kombinationstherapie eingesetzt werden können: Thiaziddiuretika, ACE-Inhibitoren, AT1-Antagonisten, Beta-Blocker, Alpha-Blocker, Renininhibitoren und Kalziumantagonisten. Die Wahl des Medikaments ist abhängig von kardiovaskulären Risikofaktoren des Patienten, Endorganschäden sowie möglichen pharmakologischen Interaktionen mit bereits bestehender Medikation. Für weitere Behandlungsempfehlungen sei auch hier auf die einschlägigen Leitlinien verwiesen (Deutsche Gesellschaft für Hypertonie und Prävention 2008).

\subsubsection{Versorgungsqualität der Tabakabhängigkeit in Deutschland}

Die deutsche SNICAS ${ }^{8}$-Studie ergab, dass insgesamt $56 \%$ aller als Raucher dokumentierten Patienten bereits einmal diesbezüglich beraten wurden, aber nur $12 \%$ an einem Entwöhnungsprogramm teilgenommen hatten (Hoch et al. 2004). Einer aktuellen Umfrage unter Allgemeinmedizinern in Brandenburg ist zu entnehmen, dass nur 30\% aller niedergelassenen Ärzte den Raucherstatus ihrer Patienten dokumentieren und nur knapp 13\% ihre rauchenden Patienten zu diesem Thema beraten (Skoeries et al. 2010). Eine jüngst publizierte Untersuchung zeigt, dass auch im Rahmen einer stationären Behandlung lediglich einem Drittel aller befragten rauchenden Patienten von ihren behandelnden Ärzten ein Rauchstopp empfohlen wurde (Raupach et al. 2011). Ursachen hierfür liegen neben Zeit- und Vergütungsmangel (Jaen et al. 1994, Pine et al. 1999, Conroy et al. 2005, Williams et al. 2003) nicht zuletzt in unzureichender Ausbildung (Britton et al. 2001, Chatkin J und Chatkin G 2009, Pederson et al. 2006, Springer et al. 2008). Entsprechend sieht sich nur ein Drittel der niedergelassenen Allgemeinmediziner in der Lage, Raucher kompetent zu beraten (Twardella und Brenner 2005).

\subsubsection{Versorgungsqualität der Alkoholabhängigkeit in Deutschland}

Ergebnissen der $\operatorname{TACOS}^{9}$-Studie zufolge werden weniger als die Hälfte aller therapiebedürftigen Alkoholprobleme als solche erkannt und weniger als ein Drittel aller alkoholabhängigen Patienten behandelt (Rumpf und Meyer 2000); lediglich 3\% werden in speziali-

\footnotetext{
${ }^{8}$ SNICAS $=$ Smoking and Nicotine Dependence Awareness and Screening Study

${ }^{9}$ TACOS $=$ Transitions in Alcohol Consumption and Smoking
} 
sierten Suchtzentren therapiert (Mann 2002). In Allgemeinkrankenhäusern erhielten insgesamt nur 9\% aller alkoholabhängigen Patienten spezifische Hilfen bezüglich der zugrunde liegenden Alkoholproblematik (John et al. 1996).

\subsubsection{Versorgungsqualität des Diabetes mellitus in Deutschland}

Eine Analyse der Daten aus dem Bundesgesundheitssurvey 1998 ergab, dass etwa ein Viertel der diagnostizierten Diabetiker mit Insulin therapiert wird und 46\% der Patienten mit oralen Antidiabetika behandelt werden. Während bei weiteren 13\% diätetische Maßnahmen zur Therapie des Diabetes mellitus eingesetzt werden, bleiben knapp 17\% ganz ohne Behandlung (Thefeld 1999). In einer Querschnittsuntersuchung aus dem Jahr 2005 betrug die Zahl der diagnostizierten, aber nicht behandelten Diabetiker nur noch $8,4 \%$. Begleitend fiel jedoch auf, dass die Behandlungshäufigkeit vom gleichzeitigen Vorliegen mikro- oder makrovaskulärer Komplikationen abhing. So wurden Diabetiker ohne Symptome nur in 59,7\% der Fälle medikamentös behandelt, wohingegen Patienten mit mikrobeziehungsweise makrovaskulären Komplikationen in 63,9\% respektive 89,4\% der Fälle therapiert wurden (Lehnert et al. 2005). Weitere Hinweise auf eine unzureichende Therapiesituation hinsichtlich Behandlungshäufigkeit und Behandlungsgüte resultieren aus Ergebnissen der DIG ${ }^{10}$ - Studie (Ott et al. 2009). Hier hatten sich die Blutzuckerwerte von 4020 Typ-2-Diabetikern innerhalb eines vierjährigen Beobachtungszeitraums trotz ärztlicher Behandlung weiter verschlechtert. Während zu Beginn der Studie 42\% der Patienten die von der Deutschen Diabetes Gesellschaft vorgegebenen Zielwerte ( $\mathrm{HbA} 1 \mathrm{c} \leq 6,5 \%)$ erreichten, waren es am Studienende nur noch $37 \%$.

\subsubsection{Versorgungsqualität der arteriellen Hypertonie in Deutschland}

Gemäß einer Studie aus dem Jahr 2003 ist die Versorgungsqualität der arteriellen Hypertonie in weiten Teilen Europas, insbesondere in Deutschland, noch ungenügend (WolfMaier et al. 2003). Aktuelleren Publikationen sind unterschiedliche Angaben zur Versorgungsqualität zu entnehmen: Folgt man Prugger et al., werden lediglich 26\% aller Hypertoniker behandelt und nur in $8 \%$ der Fälle normotone Blutdruckwerte erreicht. Die Auswertung verschiedener deutscher Querschnittsstudien aus dem Jahr 2006 ergab, dass 26$31 \%$ der männlichen und $44-48 \%$ der weiblichen Patienten mit bekannter arterieller Hypertonie behandelt werden (Löwel et al. 2006); weniger als die Hälfte der behandelten Hypertoniker war jedoch normoton. Nach Sharma et al. werden von allen objektiv diagnostizierten Hypertonikern insgesamt $64 \%$ behandelt und knapp ein Fünftel ist gut einge-

${ }^{10}$ DIG = Diabetes in Germany 
stellt. In einer neueren Untersuchung von 2934 Hausarztpraxen konnte die Erreichung des Zielblutdrucks bei knapp einem Viertel $(23,3 \%)$ der Patienten dokumentiert werden (Lemmer et al. 2008).

\subsection{Ausbildungssituation}

Die Diskrepanz zwischen Relevanz der oben genannten Erkrankungen und deren Versorgungsqualität lässt zwangsläufig die Frage aufkommen, ob Ärzte im Hinblick auf Theorie und Behandlung der Erkrankungen adäquat ausgebildet werden.

Die Studienlage zur Ausbildungssituation von arterieller Hypertonie und Diabetes mellitus ist relativ dünn. Eine Studie aus dem Jahr 1993 zeigte, dass sich Studenten im Umgang mit Diagnose und Therapie der arteriellen Hypertonie signifikant sicherer fühlten als im Umgang mit Alkoholabhängigkeit (Lasswell et al. 1993).

Im Jahr 1997 wiesen McCord et al. auf Lücken in der medizinischen Ausbildung zur Behandlung von Patienten mit Diabetes hin. Aus einer Befragung von Medizinabsolventen in Großbritannien ging hervor, dass sich nach dem Studium lediglich $28 \%$ der Befragten in der Lage fühlten, einen Diabetes richtig zu diagnostizieren. Weniger als die Hälfte der Befragten gab an, regelmäßig eine Blutzuckereinstellung bei ihren Patienten vorzunehmen (George et al. 2008). In einer aktuellen Studie aus Pakistan waren theoretische Kenntnisse zu Krankheitsaspekten des Diabetes mellitus bei einem Großteil der Studenten vorhanden (Mumtaz et al. 2009). Daten aus Deutschland fehlen nach aktueller Literaturdurchsicht.

Besser untersucht ist die Ausbildungssituation zur Tabakabhängigkeit. Richmond et al. zeigten im Jahr 1998 im Rahmen einer weltweiten Untersuchung, dass lediglich 11\% der befragten Universitäten über einen eigenen Tabak-Kurs verfügten und nur ein Drittel der Universitäten neben Rauch-assoziierten Erkrankungen auch über Tabakentwöhnungsmaßnahmen sprach. An zwölf Prozent der Universitäten wurde die Thematik gar nicht behandelt. Zehn Jahre später war der Anteil der Universitäten mit eigenem Tabak-Modul zwar auf $27 \%$ gestiegen, jedoch wurde an den meisten Universitäten weiterhin vorrangig Faktenwissen gelehrt (Richmond et al. 2009). Aus einer Befragung amerikanischer Universitäten ging hervor, dass Tabakentwöhnung nur in knapp 13\% der Fälle praktisch (mit Patienten) unterrichtet wurde (Ferry et al. 1999).

Bereits Ende der Achtziger Jahre wurden weltweit Studentenbefragungen zum Thema Rauchen durchgeführt. Darin wurde deutlich, dass viele rauchassoziierte Risiken selbst gegen Ende des Medizinstudiums von studentischer Seite unterschätzt wurden (Tessier et al. 1989, Tessier et al. 1992a, Tessier et al. 1992 b, Tessier et al. 1993). Zudem wurde gezeigt, dass der eigene Rauchstatus Einfluss auf das Praxisverhalten hat; rauchende 
Medizinstudenten gaben sehr viel seltener als Nichtraucher an, auch einen asymptomatischen Raucher beraten zu wollen. Zu ähnlichen Ergebnissen kam eine Studie in Australien (Richmond und Kehoe 1997). Eine Untersuchung von New Yorker Medizinstudenten im letzten Studienjahr zeigte, dass knapp zwei Drittel der Studenten ihre Ausbildung in Bezug auf Tabakentwöhnung für unzureichend hielten (Springer et al. 2008). In Großbritannien fühlten sich sechs von zehn Absolventen medizinischer Hochschulen durch ihr Studium nicht darauf vorbereitet, eine leitliniengerechte Tabakentwöhnung durchzuführen; lediglich $17 \%$ fühlten sich in der Lage, den Nutzen einer Nikotin-Ersatz-Therapie richtig zu erklären (Roddy et al. 2004). Aus Daten des Global Health Professionals Surveys (einer weltweiten Befragung von Studierenden und Auszubildenden in Gesundheitsberufen) geht hervor, dass nur $5-37 \%$ der Befragten (3. Studien-/Ausbildungsjahr) jemals in Bezug auf Raucherberatung und Tabakentwöhnung ausgebildet worden sind (GTSS ${ }^{11}$ Collaborative Group 2006).

Auch in Deutschland wurden in den Bereichen Tabakmissbrauch und Tabakentwöhnung erhebliche Defizite hinsichtlich theoretischer und praktischer Fertigkeiten identifiziert (Raupach et al. 2009). Die Aussagekraft der Studie blieb jedoch begrenzt, da nur ein Standort untersucht wurde.

Zur Ausbildungssituation des Themas „Alkoholabhängigkeit“ existieren ebenfalls einige Studien. Untersuchungen zur curricularen Verankerung stammen größtenteils aus Großbritannien, Nordamerika und Australien. Eine Befragung von 28 englischen Universitäten ergab, dass die Hälfte der Fakultäten Unterricht zu Aspekten der Alkoholabhängigkeit hielt und der Lernerfolg der Studenten nur in $21 \%$ der Fälle überprüft wurde (Glass 1988). In kanadischen Curricula waren insbesondere die Inhalte „Diagnose und Therapie der Alkoholabhängigkeit“ nicht genügend abgedeckt (Brewster et al. 1990). Nach Einführung eines nationalen Programms zur Verbesserung der Lehrsituation konnte in Amerika und Australien auch eine verstärkte Integration alkoholbezogener Inhalte in medizinische Curricula beobachtet werden (Fleming et al. 1994, Roche 1992).

Bei der Untersuchung kanadischer Examina fiel auf, dass Studenten theoretische Defizite hatten, wenn es um das Thema Alkoholentwöhnung ging (Kahan et al. 2006). In einer zwei Jahre später durchgeführten Online-Befragung in Ontario bestätigten sich diese Wissenslücken (Midmer et al. 2008). In einer Longitudinalstudie von neuseeländischen Medizinstudenten wurde festgestellt, dass sich die Studenten mit fortschreitendem Ausbildungsstand immer weniger dazu in der Lage fühlten, alkoholabhängige Patienten zu beraten (Cape et al. 2006).

\footnotetext{
${ }^{11}$ GTSS = Global Tobacco Surveillance System
} 
In Deutschland kamen bereits im Jahr 1995 Mann und Kapp zu dem Schluss, dass Ärzte durch ihr Studium und ihre Weiterbildung in Deutschland kaum auf den Umgang mit alkoholabhängigen Patienten vorbereitet sind.

\subsection{Studienziele}

Die Prävalenz- und Komorbiditätsraten der oben genannten Risikofaktoren und Erkrankungen veranschaulichen eindringlich die hohe Belastung sowohl für die Betroffenen als auch für das gesamte Gesundheitssystem; ihre Prävention und Behandlung sollten von höchster Priorität sein. Vor allem fehlende medizinische Ausbildung führt jedoch zu der noch unzureichenden Versorgungsqualität in diesem Sektor. Daten zur curricularen Verankerung der Erkrankungen und zu studentischen Kenntnissen liegen zum größten Teil nur aus dem Ausland vor oder stammen aus begrenzten Stichproben. Zudem hat keine Studie bislang die curriculare Verankerung von Lernzielen und den Wissensstand der Studenten gleichzeitig untersucht.

Ziel der vorliegenden Arbeit war es daher, Daten von möglichst vielen deutschen medizinischen Fakultäten zu folgenden Punkten zu erheben:

1. Curriculare Verankerung von arterieller Hypertonie, Diabetes mellitus sowie Tabak- und Alkoholabhängigkeit

2. Demographie und Rauchverhalten der Studenten

3. Studentische Kenntnisse zu Komorbidität und Behandlungsoptionen von arterieller Hypertonie, Diabetes mellitus sowie Tabak- und Alkoholabhängigkeit

4. Studentische Kenntnisse zu Entwöhnungsmaßnahmen und Beratungsstrategien auf dem Gebiet der Tabak- und Alkoholentwöhnung

5. Studentisches Verhalten im Umgang mit rauchenden Patienten und selbstwahrgenomme Beratungskompetenz

6. Studentische Evaluation der eigenen Lehrsituation und Verbesserungswünsche.

Zum besseren Vergleich der Ergebnisse von Punkt 1 sollen die Daten zu Tabak- und Alkoholabhängigkeit denen zu arterieller Hypertonie und Diabetes mellitus gegenübergestellt werden. Dasselbe Procedere soll auch auf die Ergebnisse von Punkt 4 angewandt werden. Es wird hierbei vermutet, dass chronische Suchterkrankungen nicht mit der gleichen Intensität unterrichtet werden wie „klassische“ internistische Erkrankungen, die einer vergleichsweise einfachen pharmakologischen Therapie zugänglich sind. 


\section{Material und Methoden}

\subsection{Studiendesign}

Im Rahmen der vorliegenden Arbeit wurden zwei verschiedene Fragebögen eingesetzt. Ein Fragebogen zur curricularen Verankerung von arterieller Hypertonie, Diabetes mellitus, Tabak- und Alkoholabhängigkeit wurde an die Dekanate aller deutschen medizinischen Hochschulen geschickt. Der Dekan oder ein entsprechender Lehrbeauftragter wurden darin jeweils gebeten, die curriculare Verankerung des jeweiligen Lernziels durch Angaben zur regulären Unterrichtszeit, der angewandten Lehrformate und Prüfungsformen zu präzisieren.

Mit dem zweiten Fragebogen sollten im Rahmen einer großen Studierendenbefragung medizinstudentische Kenntnisse zu allen vier Erkrankungen erhoben werden.

Während Konzeption, Produktion und Auswertung der Fragebögen im Verantwortungsbereich der Studienleitung lagen, sollte die Befragung an den jeweiligen Studienzentren mit Hilfe lokaler Ansprechpartner aus dem Studiendekanat oder der Fachschaft durchgeführt werden. Finanziell wurde die Studie als „Investigator sponsored trial“ von der Firma Johnson \& Johnson mit einem Fördervolumen von $10.000 €$ unterstützt, wobei jedoch jeglicher Einfluss auf Studiendesign, Auswertung und Nutzung der Daten vertraglich ausgeschlossen wurde. Die Genehmigung der Studie durch die zuständige Ethikkommission (Antragsnummer: 24/2/09) lag vor.

\subsection{Studienkollektiv}

Das Studium der Humanmedizin wird in Deutschland von 36 Fakultäten angeboten. Neben einem traditionell in drei Abschnitte (Vorklinik, Klinik, Praktisches Jahr) gegliederten Regelstudiengang existieren sogenannte Modellstudiengänge, deren Schwerpunkt auf einer Vereinheitlichung von Vorklinik und Klinik basiert, wodurch ein praxis- und patientennäheres Studium geschaffen werden soll. Modellstudiengänge werden insgesamt von 6 Universitäten in Deutschland angeboten, wobei 2 Fakultäten (Bochum, Berlin) den Modellstudiengang parallel zum Regelstudiengang führen.

Zielgruppe der Studie waren alle deutschen Medizinstudenten, die sich zum Zeitpunkt der Befragung in den ersten fünf Studienjahren der Ausbildung befanden. Studierende des Praktischen Jahres waren zwecks schlechter Erreichbarkeit (die Studenten werden ganztägig in den universitätszugehörigen Lehrkrankenhäusern eingesetzt) von der Befragung ausgeschlossen. 


\subsection{Generierung des Fragebogens}

Auf Grundlage der 2006 durchgeführten Untersuchung (Raupach et al. 2009) wurde in Zusammenarbeit mit Herrn Dr. med. T. Raupach (Universitätsmedizin Göttingen), Herrn Dr. med. N. Schneider (Deutsches Krebsforschungszentrum, Heidelberg), Herrn PD Dr. Dipl.-Psych. H. Krampe (Klinik für Anästhesiologie und Intensivmedizin, Charité Berlin) und Herrn Prof. Dr. R. West (Cancer Research UK Health Behaviour Unit, University College London) ein der Fragestellung entsprechender, zweiseitiger Papierfragebogen konzipiert. Im Oktober 2008 wurde der Fragebogen an 177 Studenten des dritten klinischen Semesters in Göttingen pilotiert und optimiert.

Da nicht alle Fakultäten über ein elektronisches Umfragesystem verfügten, wurde die Option einer Digitalisierung des Fragebogens im Sinne methodischer Homogenität verworfen. Gestützt wurde diese Entscheidung durch eine Vielzahl an Studien, die in elektronischen Befragungen auf ein zum Papierstandard verändertes Antwortverhalten der Probanden hinweisen (Vallejo et al. 2007) und nachweislich schlechtere Rücklaufquoten elektronischer Umfragen (Kongsved et al. 2007) gezeigt haben.

\subsection{Aufbau des Fragebogens}

Der Fragebogen bestand insgesamt aus 30 geschlossenen Fragekomplexen, die im Folgenden noch detailliert vorgestellt werden. Auf Freitextfelder wurde angesichts des hohen erwarteten Rücklaufes und des Fehlens automatisierter Texterkennung verzichtet. Inhaltlich setzte sich der Fragebogen aus 6 Schwerpunkten zusammen.

\subsubsection{Demographie und Rauchverhalten}

In einem ersten Teil wurden anhand von sechs Fragen des Typs Einfachantwort demographische Daten wie Alter, Geschlecht und Studienjahr, sowie Angaben zum Raucherstatus der Studierenden und deren Einstellung gegenüber öffentlichen Rauchverboten erhoben.

Zusätzlich zum aktuellen Raucherstatus, bei dem zwischen regelmäßigen Rauchern (täglicher Tabakkonsum), Gelegenheitsrauchern (kein täglicher Tabakkonsum) und Nichtrauchern unterschieden wurde, wurde der Anteil der Ex-Raucher mittels „100-ZigarettenKriterium“ (Bondyl et al. 2009) bestimmt. Demzufolge muss ein Ex-Raucher in seinem Leben mindestens 100 Zigaretten geraucht haben, darf aktuell aber nicht mehr rauchen. Auf diese Weise konnte der Anteil der Nie-Raucher in der Gruppe der Nichtraucher - zu denen sich auch die Ex-Raucher zählten - ermittelt werden. 


\subsubsection{Komorbidität und Behandlung ausgewählter Erkrankungen}

Darüber hinaus wurden die Studenten zur Selbsteinschätzung ihres Kenntnisstandes in Bezug auf Komplikationen und Therapie der Tabak- und Alkoholabhängigkeit sowie der arteriellen Hypertonie und des Diabetes mellitus aufgefordert. Hierzu sollten sie die Aussagen „Ich kenne die Folgeerkrankungen der beschriebenen Erkrankung“ und „Ich weiß, wie die folgende Krankheit behandelt wird“ auf einer sechsstufigen Likert-Skala von „trifft voll zu“ bis "trifft überhaupt nicht zu“ bewerten (siehe Abbildung 1).

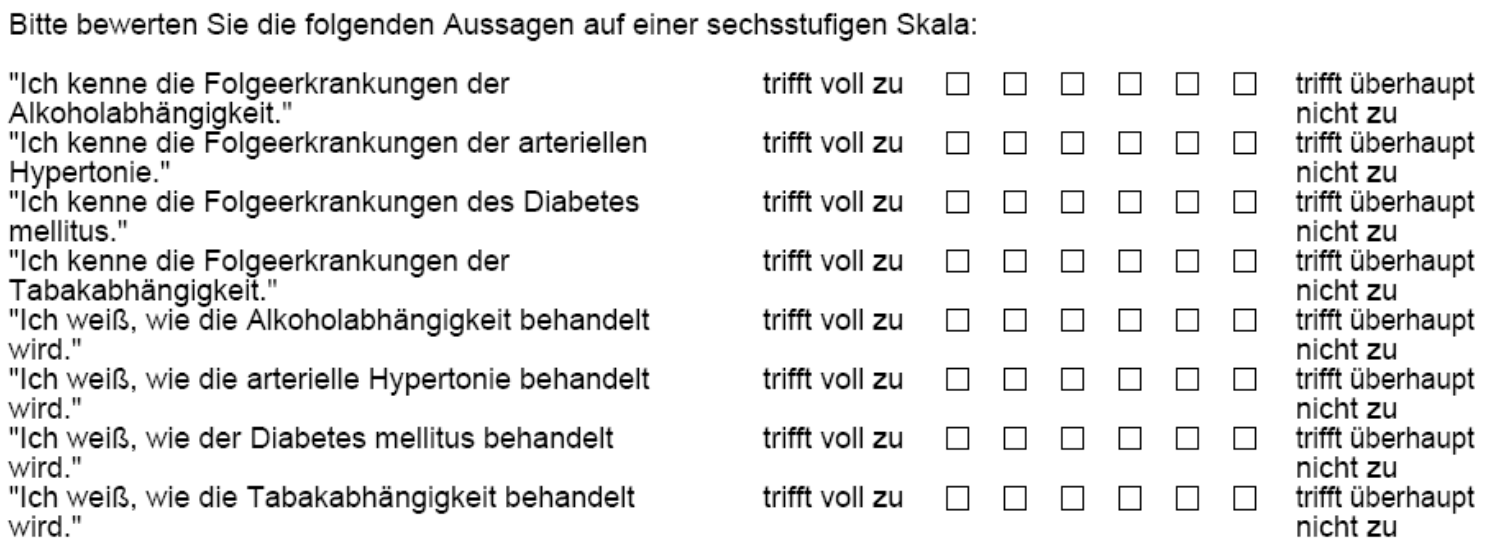

Abbildung 1. Studentische Selbsteinschätzung hinsichtlich der Komplikationen und Behandlungsoptionen von Alkohol- und Tabakabhängigkeit, sowie arterieller Hypertonie und Diabetes mellitus

\subsubsection{Entwöhnungsmaßnahmen und Beratungsstrategien}

Im Zentrum des zweiten Fragebogenteils stand die Untersuchung theoretischer Kenntnisse der Studierenden.

Dazu sollten zunächst unterschiedliche Entwöhnungsmaßnahmen der Tabakabhängigkeit hinsichtlich ihrer Wirksamkeit auf einer sechsstufigen Skala von "sehr effektiv" bis "überhaupt nicht effektiv“ beurteilt werden (siehe Abbildung 2). Im Fragentext wurde eine „effektive" Entwöhnungsmaßnahme durch eine Abstinenzquote von 30\% nach einem Jahr charakterisiert. Studierende, die keine Einschätzung zur Effektivität abgeben wollten, konnten das Feld „keine Angabe“ wählen. 
Wenn ein Jahr nach einer Maßnahme zur Tabakentwöhnung noch ca. 30\% aller Teilnehmer "abstinent" sind, bezeichnet man diese Maßnahme als "sehr effektiv". Wie würden Sie - vor diesem Hintergrund - die Effektivität folgender Entwöhnungs-Maßnahmen einschätzen?

a) Willenskraft allein

sehr effektiv

b) Beratungsgespräch mit dem Hausarzt

c) Nikotin-Ersatz-Therapie

d) Antidepressiva

e) partielle nACh-Rezeptor-Agonisten

f) Selbsthilfe-Literatur

g) Akupunktur

h) kombiniertes Entwöhnungsprogramm

(Gruppensitzungen plus Nikotin-Ersatz-

Therapie)

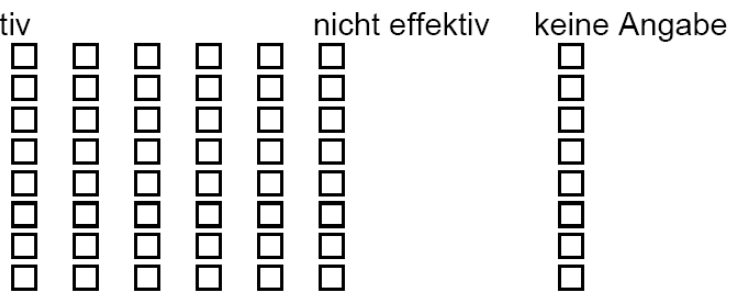

Abbildung 2. Auszug aus dem für die vorliegende Arbeit genutzten Fragebogen zur Effektivität einzelner Tabakentwöhnungsmaßnahmen

Analog zur Tabakentwöhnung wurden die Studenten um Einschätzung der langfristigen Effektivität verschiedener Alkoholentwöhnungsmaßnahmen gebeten (siehe Abbildung 3).

Wenn ein Jahr nach einer Maßnahme zur Alkohol-Entwöhnung noch ca. 30\% aller Teilnehmer "abstinent" sind, bezeichnet man diese Maßnahme als "sehr effektiv". Wie würden Sie - vor diesem Hintergrund - die Effektivität folgender Entwöhnungs-Maßnahmen einschätzen?
a) Willenskraft allein
b) Beratungsgespräch mit dem Hausarzt
c) sogenannter "Turbo-Entzug"
d) Selbsthilfe-Gruppen
e) Selbsthilfe-Literatur
f) Akupunktur
g) Entzugstherapie in einer Klinik (14 Tage)
h) Entzugstherapie mit anschließender
Entwöhnungsbehandlung sehr effektiv

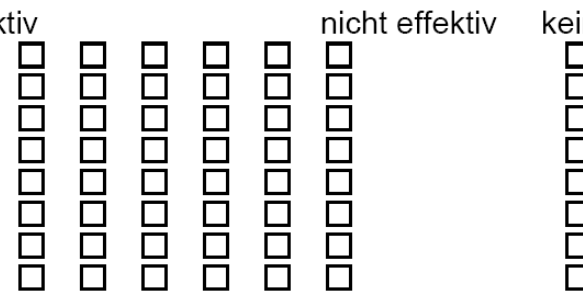

Abbildung 3. Auszug aus dem für die vorliegende Arbeit genutzten Fragebogen zur Effektivität einzelner Alkoholentwöhnungsmaßnahmen

Hierbei waren ebenfalls acht Vorgaben auf einer sechsstufigen Skala von „sehr effektiv“ bis „überhaupt nicht effektiv“ zu beurteilen. Des Weiteren sollten die Studenten auf einer Skala von 0 bis 5 angeben, wie viele der in der Raucherberatung als „5 A“ bezeichneten Maßnahmen sie tatsächlich explizit benennen können. Auf gleiche Weise sollten die Studierenden auf einer Skala von 0 bis 6 kennzeichnen, wie viele der im Akronym „FRAMES“ (Miller WR und Sanchez 1994) zusammengefassten Maßnahmen sie benennen können.

\subsubsection{Praxisverhalten und Beratungskompetenz}

In Form zweier dichotomer Fragen wurde untersucht, mit welcher Regelmäßigkeit die Studenten den Rauchstatus ihrer Patienten erfragen, und ob sie allen Rauchern einen Rauchstopp empfehlen. Studierende des vorklinischen Abschnittes oder solche ohne kontinuierlichen Patientenkontakt hatten die Möglichkeit das Feld „Ich habe keinen regelmäßigen Patientenkontakt“ zu wählen. 
Abschließend sollten die Studenten eine Einschätzung zu ihrer selbstwahrgenommen Kompetenz zur Raucherberatung abgeben, indem sie die Aussage „Ich bin in der Lage, einen Raucher, der mit dem Rauchen aufhören möchte, kompetent zu beraten“ auf einer sechsstufigen Likert-Skala von „trifft voll zu“ bis „trifft überhaupt nicht zu“ bewerteten.

\subsubsection{Lehrsituation und Verbesserungswünsche}

In einem letzten Teil wurden die Studierenden gefragt, ob sie im Laufe ihres Studiums theoretischen und/oder praktischen Unterricht bezüglich der Tabakabhängigkeit und deren Behandlungsmöglichkeiten erfahren haben. Darüber hinaus konnten sie bei zulässiger Mehrfachauswahl angeben, welche der 4 angegebenen Erkrankungen (Tabak-, Alkoholabhängigkeit, Diabetes mellitus, arterielle Hypertonie) ihrer Meinung nach intensiver unterrichtet werden müsste.

\subsection{Kontakt der Studienzentren}

Im November 2008 wurden die Studiendekanate aller medizinischen Fakultäten schriftlich kontaktiert und zur Teilnahme an der geplanten Befragung eingeladen. Für den Fall der Teilnahme waren die Fakultäten aufgefordert, aktuelle Immatrikulationszahlen zu übermitteln. Gleichzeitig wurde um Auskunft über die curriculare Verankerung, sprich den zeitlichen Aufwand und angewandte Lehr- / Prüfungsformate der Themen Tabak- und Alkoholabhängigkeit, sowie arterielle Hypertonie und Diabetes mellitus gebeten. Von insgesamt 27 Fakultäten, die eine Teilnahme an der studentischen Befragung befürworteten, machten jedoch nur 20 Angaben zu curricularen Inhalten. Weitere 5 Fakultäten legten zwar Informationen hinsichtlich ihres Curriculums dar, schlossen eine Teilnahme an der studentischen Befragung aber aus (siehe Abbildung 4).
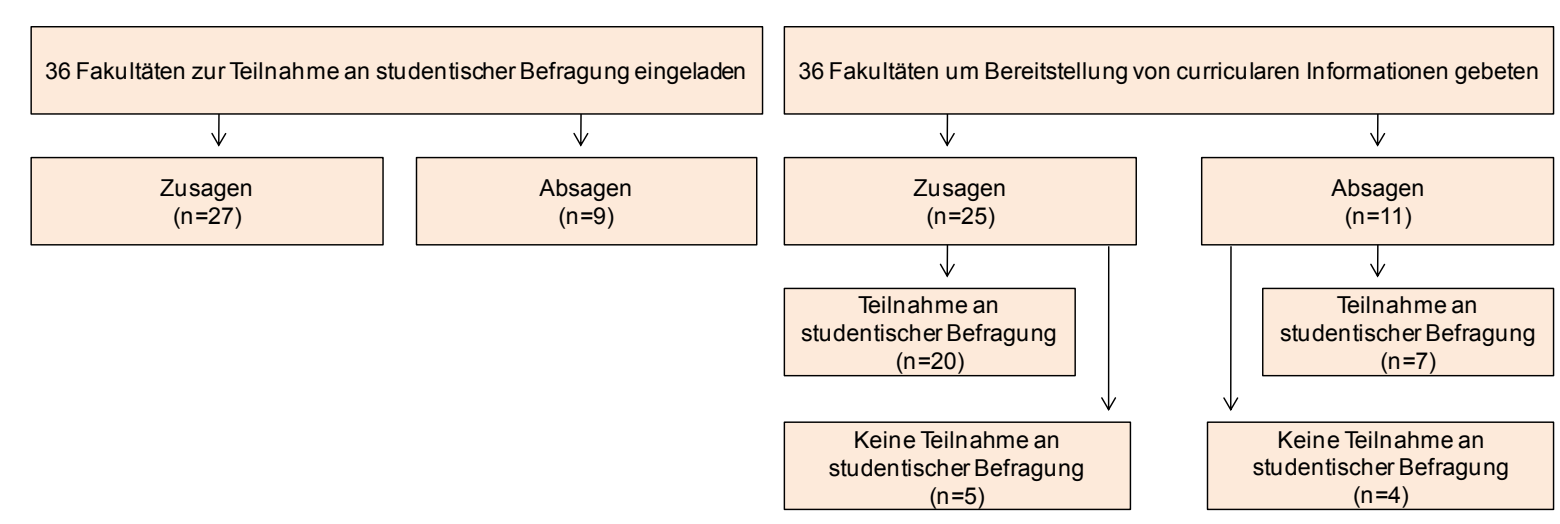

Abbildung 4. Teilnahmebereitschaft der Fakultäten 


\subsection{Studientreffen}

Im Hinblick auf eine Standardisierung der Studiendurchführung wurden im Februar 2009 alle teilnehmenden Studienzentren zu einem Studientreffen in Göttingen eingeladen. Insgesamt waren 20 Vertreter von 18 Standorten anwesend.

Hierbei bot sich neben einem persönlichen Kennenlernen die Möglichkeit des Dialogs über Studienhintergrund und Grundlage des Studiendesigns. Zugleich sollte dadurch eine Standardisierung des Studienablaufs in den einzelnen Studienzentren sichergestellt werden.

Ablauf und Diskussionsschwerpunkte des Studientreffens wurden in einem Protokoll festgehalten und an alle Studienzentren verschickt. Fakultäten, die an dem Studientreffen nicht teilnehmen konnten, wurden telefonisch kontaktiert und über wesentliche Inhalte informiert.

\subsection{Druck und Versand der Fragebögen}

Mit Hilfe des an der Universität in Göttingen genutzten Evaluationssystems Evasys ${ }^{\circledR}$ (Electric Paper, Lüneburg) wurden fakultätsspezifische Seriendrucke erstellt, die eine spätere Zuordnung der Fragebögen ermöglichten. Druck und Versand der entsprechenden Anzahl an Fragebögen wurden in Göttingen koordiniert.

\subsection{Datenerhebung}

Die Studienzentren wurden gebeten, geeignete Plenarveranstaltungen zu identifizieren, in denen eine Ausgabe der Fragebögen möglich wäre. Die Semester 1 - 9 wurden zu Beginn des Sommersemesters befragt, während das zehnte Semester erst gegen Ende des Semesters an der Papierbefragung teilnahm. Auf diese Weise sollte sichergestellt werden, dass Lerninhalte, die im Zuge des letzten Semesters vermittelt werden, nicht von der Untersuchung ausgeschlossen wurden. Als Anreiz konnte die Fakultät mit dem höchsten Rücklauf (basierend auf den zuvor schon übermittelten Immatrikulationszahlen) ein Preisgeld in Höhe von $2500 €$ gewinnen.

Im Rahmen der jeweiligen Veranstaltungen waren alle Studierenden dann zur anonymen Teilnahme an der Studie eingeladen. Nach einer kurzen Vorstellung der Studie wurden die Fragebögen an die Studierenden ausgeteilt und nach einer Bearbeitungszeit von ca. 5-10 Minuten wieder eingesammelt. 


\subsection{Datenanalyse}

Nach Abschluss der Datenerhebung wurden die Fragebögen nach Göttingen geschickt und dort zentral ausgewertet. Hierfür wurden die Fragebögen mit Unterstützung von Herrn Christian Münscher im Geschäftsbereich Informationstechnologie der Universität Göttingen gescannt. Die Rohdatenausgabe erfolgte im CSV-Format, das in gängige Statistikprogramme importierbar ist. Nach Ausschluss leerer Fragebögen wurden die Daten in Zusammenarbeit mit Prof. R. Hilgers und Prof. T. Beissbarth (Medizinische Statistik Göttingen) in SPSS 14.0 für Windows ausgewertet. Da die Fragebögen anonym und selbstständig ausgefüllt wurden, konnte kein Einfluss darauf genommen werden, dass alle Fragen vollständig beantwortet wurden. Die Gesamtstichprobe ist somit nicht für alle Items gleich groß.

Eine Sensitivitätsanalyse ergab, dass die Verwendung spezieller Einschlusskriterien (vollständige Angabe von Geschlecht, Raucherstatus und Studienjahr) keinen Einfluss auf die prinzipiellen Aussagen dieser Arbeit hatte, so dass zu Gunsten größerer Repräsentativität darauf verzichtet wurde.

Die Daten wurden zunächst im Hinblick auf den Ausbildungsfortschritt (Studienjahr) ausgewertet. Die Untersuchung auf statistische Signifikanz erfolgte in Abhängigkeit der Variableneigenschaft mittels nicht parametrischer Testverfahren (Kruskal-Wallis-H oder MannWhitney-U Test) sowie des Chi-Quadrat $\left(\chi^{2}\right)$ Tests. Um den Einfluss der Ausbildung auf den Wissensstand der Studenten hinsichtlich Folgeerkrankungen und Behandlungskompetenzen zu bewerten, wurden Spearman's Rangkorrelationskoeffezienten (Spearman's rho) berechnet. Korrelationskoeffizienten $<0,2$ bedeuten, dass das von den Studenten selbst eingeschätzte Wissen mit fortschreitender Ausbildung nicht größer ist.

Des Weiteren wurden für einzelne Variablen Subgruppenanalysen des letzten Studienjahrs nach folgenden Kriterien durchgeführt: Raucherstatus, Geschlecht und Art des Studiengangs. Hierbei wurden die Likert-Skalen zur Effektivitätseinschätzung der Tabak- und Alkoholentwöhnungsmaßnahmen dichotomisiert; die vor dem Hintergrund von Metaanalysen am ehesten zutreffende Option wurde als „richtig“ gewertet, während die restlichen fünf Optionen als „falsch“ zusammengefasst wurden. Analog wurde mit den Likert-Skalen zur Kenntnis von Folgeerkrankungen und Behandlungskompetenzen verfahren; die Optionen „trifft voll zu“ und „trifft zu“ wurden als „zutreffend“ gewertet, während die restlichen vier Optionen als „nicht zutreffend“ zusammengefasst wurden. Eine statistische Irrtumswahrscheinlichkeit von kleiner $5 \%(p<0,05)$ wurde als Signifikanzniveau festgelegt. 


\section{Ergebnisse}

\subsection{Curriculumanalyse}

Lediglich 25 medizinische Fakultäten machten Angaben darüber, in welchem zeitlichen und didaktischen Umfang arterielle Hypertonie, Diabetes mellitus sowie Tabak- und Alkoholabhängigkeit gelehrt wurden, elf Fakultäten schlossen eine Teilnahme an der Curricularbefragung aus. Zwanzig Fakultäten nahmen gleichzeitig an der Studierendenbefragung teil (siehe Abbildung 4).

In der Analyse zeigte sich, dass die Lernziele Diabetes mellitus und arterielle Hypertonie mit einer medianen Unterrichtszeit von 315 beziehungsweise 275 Minuten deutlich umfangreicher in den Curricula repräsentiert waren als Alkohol- und Tabakabhängigkeit (siehe Tabelle 9). Mit durchschnittlich 135 Minuten wurde die Tabakabhängigkeit am wenigsten intensiv unterrichtet.

Vorlesungen gehörten mit nur geringen Unterschieden zwischen den einzelnen Erkrankungen zu den häufigsten Lehrformaten (an 16-21 von 25 Fakultäten). Seminare und praxisorientierte Lehrformen, wie Praktika oder Unterricht am Krankenbett, wurden im Falle des Diabetes mellitus von ca. der Hälfte und im Falle der anderen Erkrankungen von mehr als einem Drittel der Fakultäten als Lehrformat angegeben. Einige wenige Fakultäten nutzten andere Lehransätze wie E-Learning (engl.: electronic learning = „elektronisch gestütztes Lernen“) und POL (Problem-orientiertes Lernen). Obwohl fast die Hälfte aller Fakultäten angab, praxisorientierte Lernmethoden zur Wissensvermittlung einzusetzen, berichteten lediglich 2 Fakultäten davon, den Lernerfolg auch praktisch im Rahmen eines OSCE (engl.: objective structured clinical examination) zu prüfen. In über $90 \%$ der Fälle erfolgte die Lernkontrolle schriftlich mittels Multiple-Choice-Fragen oder mündlich. 


\begin{tabular}{lc|cccc|cc|c} 
Erkrankung & $\begin{array}{c}\text { Reguläre Unterrichtszeit in } \\
\text { Minuten }\end{array}$ & \multicolumn{5}{|c|}{ Lehrformat $^{*}$} & Prüfungsform $^{*}$ \\
& Median (range) & $\begin{array}{c}\text { Vorlesung } \\
(\mathrm{n})\end{array}$ & $\begin{array}{c}\text { Seminar } \\
(\mathrm{n})\end{array}$ & $\begin{array}{c}\text { PRA } \\
(\mathrm{n})\end{array}$ & $\begin{array}{c}\text { E-Learning } \\
(\mathrm{n})\end{array}$ & $\begin{array}{c}\text { POL } \\
(\mathrm{n})\end{array}$ & $\begin{array}{c}\text { OSCE } \\
(\mathrm{n})\end{array}$ & $\begin{array}{c}\text { Andere } \\
(\mathrm{n})\end{array}$ \\
\hline Diabetes mellitus & $315(15-1210)$ & 16 & 15 & 13 & 1 & 3 & 2 & 21 \\
Arterielle Hypertonie & $275(15-1530)$ & 17 & 11 & 10 & - & 2 & 2 & 22 \\
Alkoholabhängigkeit & $180(45-1080)$ & 20 & 11 & 10 & 1 & 5 & 2 & 20 \\
Tabkabhängigkeit & $135(25-720)$ & 21 & 11 & 9 & 1 & 2 & 2 & 21 \\
\hline
\end{tabular}

Tabelle 9. Curriculare Verankerung der Lernziele Diabetes mellitus, arterielle Hypertonie, Alkohol- und Tabakabhängigkeit.

Daten lagen von insgesamt 25 Fakultäten vor; da aber nicht jede Fakultät in der Lage war, alle Fragen zu beantworten, variiert die Grundgesamtheit zwischen den einzelnen Variablen.

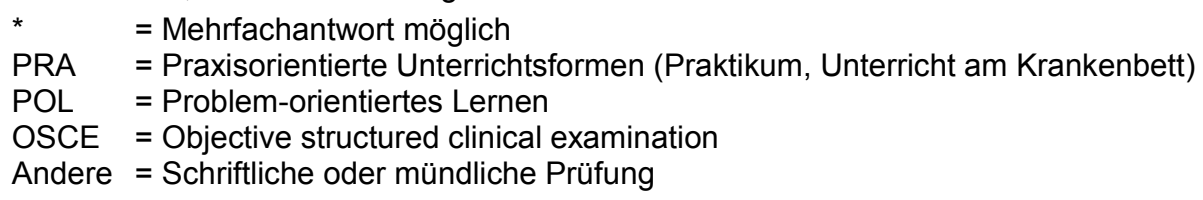

\subsection{Auswertung der Studierendenbefragung}

\subsubsection{Datengrundlage}

An der Studierendenbefragung nahmen 27 von 36 Fakultäten teil; darunter boten zwei Fakultäten (Bochum und Berlin) einen Regel- und Modellstudiengang an. Von 39.358 immatrikulierten Studenten beteiligten sich 19.973 an der Umfrage. Nach Ausschluss von 445 Fragebögen (2,2\%) aufgrund leerer Datensätze wurden weitere 19.528 Fragebögen gescannt und ausgewertet, womit sich eine absolute Rücklaufquote (entsprechend der Absolutzahl an zurückgekommenen Fragebögen in Bezug auf die studentische Gesamtzahl) von 49,6\% ergab. Die effektiven Rücklaufquoten (entsprechend der Anzahl an tatsächlich auswertbaren Fragebögen in Bezug auf die studentische Gesamtzahl) einzelner Lehrveranstaltungen waren jedoch mit $80-90 \%$ deutlich höher. Zudem variierten die Rücklaufquoten der einzelnen Studienzentren wesentlich $(1,9 \%$ bis zu $99,2 \%$, siehe Tabelle 10). 16 Fakultäten erzielten jedoch eine Rücklaufquote über $50 \%$.

\subsubsection{Demographie und Rauchverhalten}

Der Großteil der befragten Studenten studierte Medizin im Regelstudiengang (89.1\%). Fast zwei Drittel der Befragten (64,6\%) waren weiblichen Geschlechts und über die Hälfte aller Studenten $(52,6 \%)$ befand sich zum Zeitpunkt der Befragung im klinischen Abschnitt des Medizinstudiums. Über 90 \% der Studenten waren unter 27 Jahre alt. 
Tabelle 11 fasst demographische Angaben und studentische Rauchgewohnheiten zusammen. Signifikante demographische Unterschiede zwischen Studenten des Regelstudiengangs und Studenten des Modellstudiengangs wurden nicht gefunden.

Insgesamt bezeichneten sich 20,1\% der Studenten als Raucher; 9,4\% ( $n=1745)$ zählten sich zu den regelmäßigen und 10,7\% $(n=1989)$ zu den Gelegenheitsrauchern. Der Raucheranteil unter Männern lag mit 24\% signifikant höher als der Raucheranteil unter Frauen $\left(17.9 \%, \chi^{2}: p<0,001\right)$. Im Studienjahrvergleich zeigten sich keine signifikanten Unterschiede in Bezug auf die Raucherquote; weder der Anteil an regelmäßigen Rauchern $\left(9,6 \%\right.$ im 1. Studienjahr vs. $8,9 \%$ im 5. Studienjahr; $\left.\chi^{2}: p=0,419\right)$ noch der Anteil an Gelegenheitsrauchern $\left(10,2 \%\right.$ im 1 . Studienjahr vs. $10,7 \%$ im 5 . Studienjahr, $\left.\chi^{2}: p \quad 0,770\right)$ variierte wesentlich (siehe Tabelle 12). Während die Raucherquote in den einzelnen Studienjahren nahezu konstant blieb, war die Bereitschaft unter Rauchern, mit dem Rauchen aufzuhören, gegen Ende des Medizinstudiums am größten. Im ersten Jahr waren hierzu lediglich $48,3 \%$ aller Raucher bereit, im fünften Jahr betrug dieser Anteil bereits $57,1 \%\left(\chi^{2}\right.$ : $p<0,001)$.

Das am 1. Juli 2008 in Deutschland eingeführte Rauchverbot wurde von der Mehrheit der Studenten unterstützt (83,6\%). Befürwortet wurde das Rauchverbot von 90\% der Nichtraucher und von zwei Drittel der Raucher $\left(62,6 \%\right.$ vs. 88,9\%; $\chi^{2}$ : $\left.p<0,001\right)$. Im Studienjahrvergleich (siehe Tabelle 13) fällt auf, dass der Anteil derjenigen, die dem Rauchverbot positiv gegenüberstehen, gegen Ende des Studiums größer war als zu Beginn (86,8\% vs. 80,9\%; $\left.\chi^{2}: p<0,001\right)$. 


\section{Universität}

Anzahl immatrikulierter

Studenten $(n)^{\star}$
Anzahl ausgefüllter

Fragebögen ( $n$ )
Rücklaufquote

(\%)

\begin{tabular}{|c|c|c|c|}
\hline Aachen* & 1227 & 679 & 55,3 \\
\hline Berlin & 2400 & 647 & 27,0 \\
\hline Berlin* & 600 & 140 & 23,3 \\
\hline Bochum & 1021 & 747 & 73,2 \\
\hline Bochum* & 210 & 178 & 84,8 \\
\hline Bonn & 1320 & 25 & 01,9 \\
\hline Duisburg-Essen & 720 & 460 & 63,9 \\
\hline Düsseldorf & 2126 & 848 & 39,9 \\
\hline Erlangen-Nürnberg & 1483 & 857 & 57,8 \\
\hline Frankfurt am Main & 1841 & 248 & 13,5 \\
\hline Göttingen & 1843 & 1402 & 76,1 \\
\hline Greifswald & 645 & 640 & 99,2 \\
\hline Halle-Wittenberg & 1158 & 955 & 82,5 \\
\hline Hamburg & 1406 & 1096 & 78,0 \\
\hline Heidelberg* & 1535 & 142 & 9,3 \\
\hline Jena & 1290 & 793 & 61,5 \\
\hline Kiel & 1027 & 746 & 72,6 \\
\hline Köln* & 1600 & 769 & 48,1 \\
\hline Leipzig & 2200 & 757 & 34,4 \\
\hline Lübeck & 1015 & 439 & 43,3 \\
\hline Mainz & 2046 & 1004 & 49,3 \\
\hline Marburg & 1580 & 1332 & 84,3 \\
\hline München (LMU) & 2949 & 1441 & 48,9 \\
\hline München (TU) & 840 & 241 & 28,7 \\
\hline Münster & 1265 & 742 & 58,7 \\
\hline Regensburg & 844 & 562 & 66,6 \\
\hline Ulm & 1666 & 844 & 50,7 \\
\hline Witten-Herdecke* & 251 & 224 & 89,2 \\
\hline Würzburg & 1250 & 570 & 45,6 \\
\hline Gesamt & 39.358 & 19.528 & 49,6 \\
\hline
\end{tabular}

Tabelle 10. Rücklauf der einzelnen Studienzentren.

Modellstudiengänge sind mit einem * gekennzeichnet. 


\section{Studenten (n) Studenten (\%)}

\begin{tabular}{lcc}
\hline Studiengang & 19.528 & \\
Regelstudiengang & 17.396 & $89,1 \%$ \\
Modellstudiengang & 2.132 & $10,9 \%$ \\
Geschlecht & 18.500 & \\
Weiblich & 11.951 & $64,6 \%$ \\
Männlich & 6549 & $35,4 \%$ \\
Studienjahr & 18.667 & \\
1. Jahr & 4.534 & $24,3 \%$ \\
2. Jahr & 4.309 & $23,1 \%$ \\
3. Jahr & 3.550 & $19,0 \%$ \\
4. Jahr & 3.212 & $17,2 \%$ \\
5. Jahr & 3.062 & $16,4 \%$ \\
Rauchgewohnheiten & 18.532 & \\
Regelmäßige Raucher & 1.745 & $9,4 \%$ \\
Gelegenheitsraucher & 1.989 & $10,7 \%$ \\
Nichtraucher & 14.798 & $79,9 \%$ \\
\hline
\end{tabular}

Tabelle 11. Demographische Daten und Rauchcharakteristik

\begin{tabular}{|c|c|c|c|c|c|c|c|c|c|c|}
\hline & \multicolumn{2}{|c|}{$\begin{array}{c}\text { 1. Jahr } \\
(n=4.386)\end{array}$} & \multicolumn{2}{|c|}{$\begin{array}{l}\text { 2. Jahr } \\
(n=4.136)\end{array}$} & \multicolumn{2}{|c|}{$\begin{array}{c}\text { 3. Jahr } \\
(n=3.420)\end{array}$} & \multicolumn{2}{|c|}{$\begin{array}{c}\text { 4. Jahr } \\
(n=3.116)\end{array}$} & \multicolumn{2}{|c|}{$\begin{array}{c}\text { 5. Jahr } \\
(n=2.941)\end{array}$} \\
\hline & $(\mathrm{n})$ & $(\%)$ & (n) & $(\%)$ & (n) & $(\%)$ & (n) & $(\%)$ & (n) & $(\%)$ \\
\hline Raucher gesamt & 872 & 19,9 & 873 & 21,1 & 681 & 19,9 & 610 & 19,6 & 578 & 19,7 \\
\hline Regelmäßige Raucher & 423 & 9,6 & 413 & 10,0 & 311 & 9,1 & 278 & 8,9 & 263 & 8,9 \\
\hline Gelegenheitsraucher & 449 & 10,2 & 460 & 11,1 & 370 & 10,8 & 332 & 10,7 & 315 & 10,7 \\
\hline
\end{tabular}

Tabelle 12. Raucherquote in den einzelnen Studienjahren 


\begin{tabular}{|c|c|c|c|c|c|c|c|c|c|}
\hline \multicolumn{2}{|c|}{$\begin{array}{c}\text { 1. Jahr } \\
(n=4.299)\end{array}$} & \multicolumn{2}{|c|}{$\begin{array}{c}\text { 2. Jahr } \\
(n=4.041)\end{array}$} & \multicolumn{2}{|c|}{$\begin{array}{l}\text { 3. Jahr } \\
(n=3.361)\end{array}$} & \multicolumn{2}{|c|}{$\begin{array}{c}\text { 4. Jahr } \\
(n=3.054)\end{array}$} & \multicolumn{2}{|c|}{$\begin{array}{c}\text { 5. Jahr } \\
(n=2.903)\end{array}$} \\
\hline (n) & $(\%)$ & $(n)$ & $(\%)$ & (n) & $(\%)$ & (n) & (\%) & (n) & $(\%)$ \\
\hline 3.476 & 80,9 & 3.293 & 81,5 & 2.841 & 84,5 & 2.640 & 86,4 & 2.521 & 86,8 \\
\hline
\end{tabular}

Tabelle 13. Anteil der Studenten (n), die das öffentliche Rauchverbot befürworten.

\subsubsection{Komorbidität und Behandlung ausgewählter Erkrankungen}

Abbildung 5 A vergleicht das studentische Wissen zu Folgeerkrankungen von Tabakund Alkoholabhängigkeit in allen fünf Studienjahren mit dem von arterieller Hypertonie und Diabetes mellitus. Als Zustimmung wurden die Optionen „trifft voll zu“ und "trifft zu“ gewertet, die im Bild durch die Farben Grün und Hellgrün dargestellt sind. Im ersten Studienjahr gaben mehr als die Hälfte der Studenten an, die Folgeerkrankungen der Tabakund Alkoholabhängigkeit zu kennen; gegen Ende des Medizinstudiums betrug dieser Anteil knapp $80 \%$. Für die arterielle Hypertonie und den Diabetes mellitus ergaben sich im ersten Studienjahr ähnliche Zahlen, im letzten Studienjahr betrug der Anteil der Studenten, die behaupteten, die Folgeerkrankungen dieser Pathologien zu kennen, jedoch etwas über $90 \%$. Weiterhin zeigte sich, dass die Korrelationen zwischen studentischen Angaben und Ausbildungsstand in den Bereichen arterielle Hypertonie (Spearman's rho $=0,47, p<$ 0,001 ) und Diabetes mellitus (Spearman's rho $=0,42, p<0,001$ ) größer sind als in den Bereichen Tabak- (Spearman's rho $=0,19, p<0,001$ ) und Alkoholabhängigkeit (Spearman's rho $=0,24, p<0,001)$.

Ein etwas anderes Bild zeichnete sich für die Behandlungsoptionen der einzelnen Erkrankungen ab (Abbildung 5 B). Als Zustimmung wurden ebenfalls die Optionen "trifft voll zu“ und "trifft zu“ gewertet. Zu Beginn des Studiums gaben circa 20\% der Studenten an zu wissen, wie der Diabetes mellitus therapiert wird, bei allen anderen Erkrankungen waren es nur knapp 10\%. Im letzten Studienjahr betrug der Anteil der Studenten, die die Behandlungsmöglichkeiten der arteriellen Hypertonie und des Diabetes mellitus zu kennen glaubten, circa $80 \%$. Anders verhielt es sich für die Tabak- und Alkoholabhängigkeit; hier blieb der Anteil der Studenten, die nach eigenen Angaben die therapeutischen Ansätze zur Behandlung kannten, in allen Studienjahren konstant niedrig. Analog zu Abbildung 5 A war der Wissenszuwachs in den Bereichen arterielle Hypertonie (Spearman's rho = $0,55, p<0,001$ ) und Diabetes mellitus (Spearman's rho $=0,40, p<0,001$ ) sehr viel größer als in den Bereichen Tabak- (Spearman's rho $=0,04, p<0,001$ ) und Alkoholabhängigkeit (Spearman's rho $=0,15, p<0,001)$. 


\subsubsection{Entwöhnungsmaßnahmen und Beratungsstrategien}

Abbildung 6 stellt die Einschätzungen der Studenten von verschiedenen Tabakentwöhnungsmaßnahmen dar. Auf der y-Achse ist hierbei einerseits der prozentuale Anteil der Studenten angegeben und andererseits eine Effektivitätsskala von „hoch effektiv" bis „nicht effektiv“ abgebildet. Als „hoch effektiv“ wurde im Fragentext eine 1-JahresAbstinenzquote von 30\% definiert. Auf der x-Achse sind die einzelnen Studienjahre aufgetragen. Die Stufe der Likert-Skala, die am ehesten den Angaben publizierter MetaAnalysen entspricht, ist in der Abbildung blau dargestellt und damit der Anteil der Studenten, der die Effektivität der jeweiligen Maßnahme richtig eingeschätzt hat. Die grauen Bereiche stellen den Anteil der Studenten dar, die die Effektivität der Maßnahme über- beziehungsweise unterschätzt haben.

In der Tabakentwöhnung wiesen knapp 30\% der Studenten „Willenskraft allein“ eine hohe Effektivität zu, gefolgt von „Kombiniertes Entwöhnungsprogramm“ $(23,8 \%)$ und mit etwas Abstand „Akupunktur“ (4.3\%) und „Nikotinersatztherapie“ (4.2\%). Diese Einschätzungen unterschieden sich in den einzelnen Studienjahren kaum. Die Effektivität von hausärztlicher Beratung und der Stellenwert von Antidepressiva in der medikamentösen Therapie der Raucherentwöhnung hielten die Studenten für gering. Die besonders hohe Anzahl an „Missing Values“ für das Item „nikotinerge Acetylcholinrezeptor-Agonisten“ lässt vermuten, dass die Studenten dieses noch recht neue Prinzip der Tabakentwöhnung entweder nicht kannten oder die Begrifflichkeit nicht verstanden haben.

Die Effektivität der einzelnen Alkoholentwöhnungsmaßnahmen wurde insgesamt zwar etwas besser, aber dennoch von nur einem geringen Teil der Studenten richtig eingeschätzt (siehe Abbildung 7). Die Mehrzahl der Studenten (40,8\%) wies dem stationären Entzug mit anschließender Entwöhnungstherapie eine hohe Effektivität zu. Die Effektivität der hausärztlichen Beratung und die Bedeutsamkeit von Selbsthilfegruppen wurden gering eingestuft, während die Effektivität von „Willenskraft allein“ von fast einem Viertel der Studenten (21,3\%) als besonders hoch eingeschätzt wurde. In Abbildung 7 wird zudem deutlich, dass sich die Einschätzungen zur Effektivität selbst mit fortschreitendem Ausbildungsstand kaum verändern.

Insgesamt gaben lediglich 373 von 19.528 Studenten an, alle fünf Einzelmaßnahmen der in der Raucherberatung als „5A“ bekannten Behandlungsstrategie benennen zu können. Bei Betrachtung einzelner Studienjahre zeigt sich, dass der Anteil der Studenten, der mindestens eine Maßnahme benennen konnte, zwar mit 26,6\% im ersten Studienjahr und $32,9 \%$ im letzten Studienjahr signifikant größer war (Kruskal-Wallis- $\mathrm{H}: \mathrm{p}<0,001$ ), aber selbst gegen Ende des Medizinstudiums nur 3,2\% der Studenten die Bedeutung jedes einzelnen der „5A“ kannten (siehe Tabelle 14). Ähnlich schlecht waren die studentischen 
Kenntnisse über Vorgehensweisen in der Beratung alkoholabhängiger Patienten (FRAMES). Insgesamt gaben weniger als ein Fünftel der Studenten an, alle sechs Maßnahmen explizit benennen zu können. Zwar war auch der Anteil der Studenten, der mindestens eine Maßnahme kannte, im letzten Studienjahr signifikant größer als im ersten $(21,7 \%$ vs. 16,5\%; Kruskal-Wallis-H: $\mathrm{p}<0,001$ ), dennoch blieb der Anteil gering (siehe Tabelle 15). 

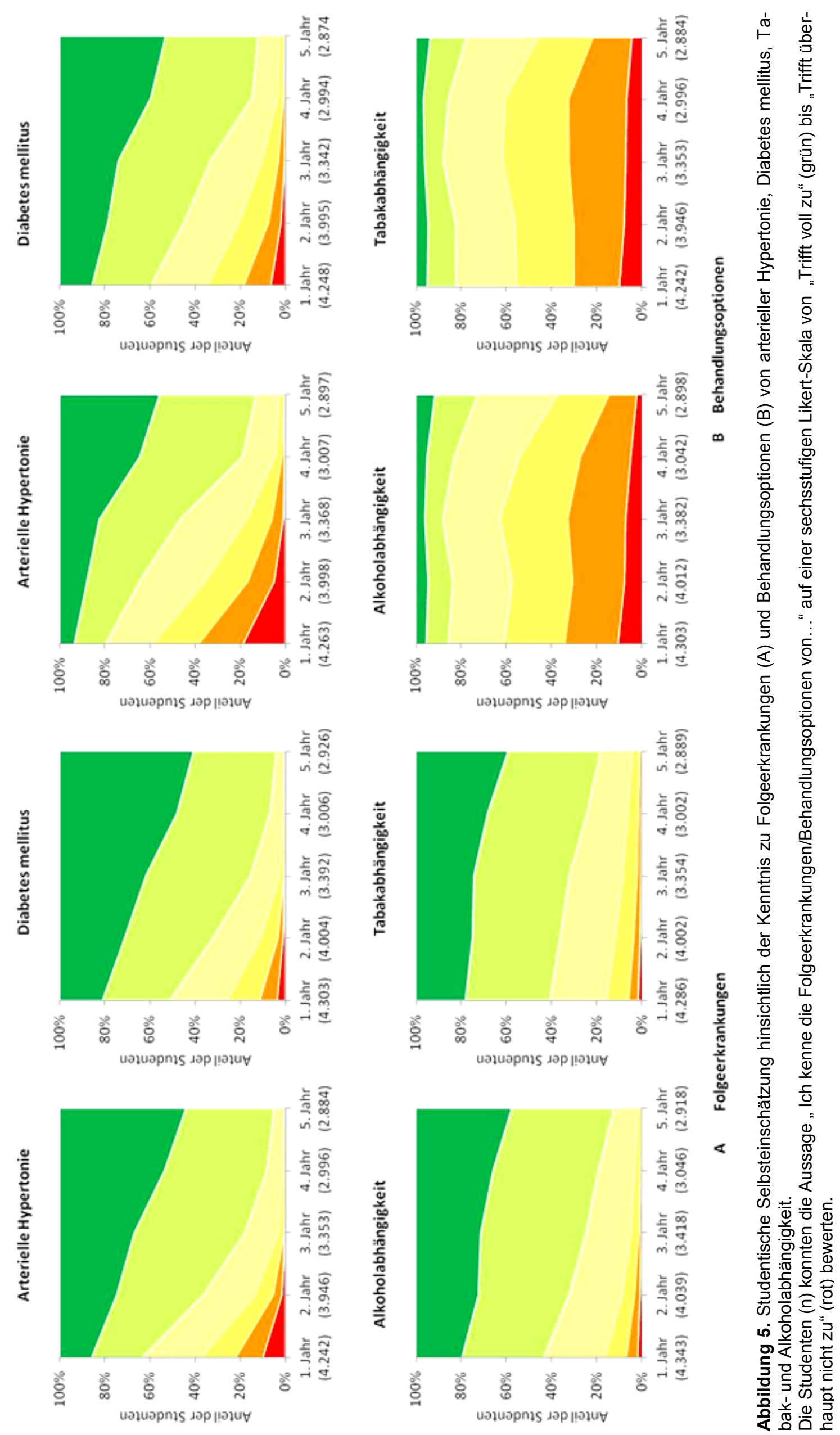

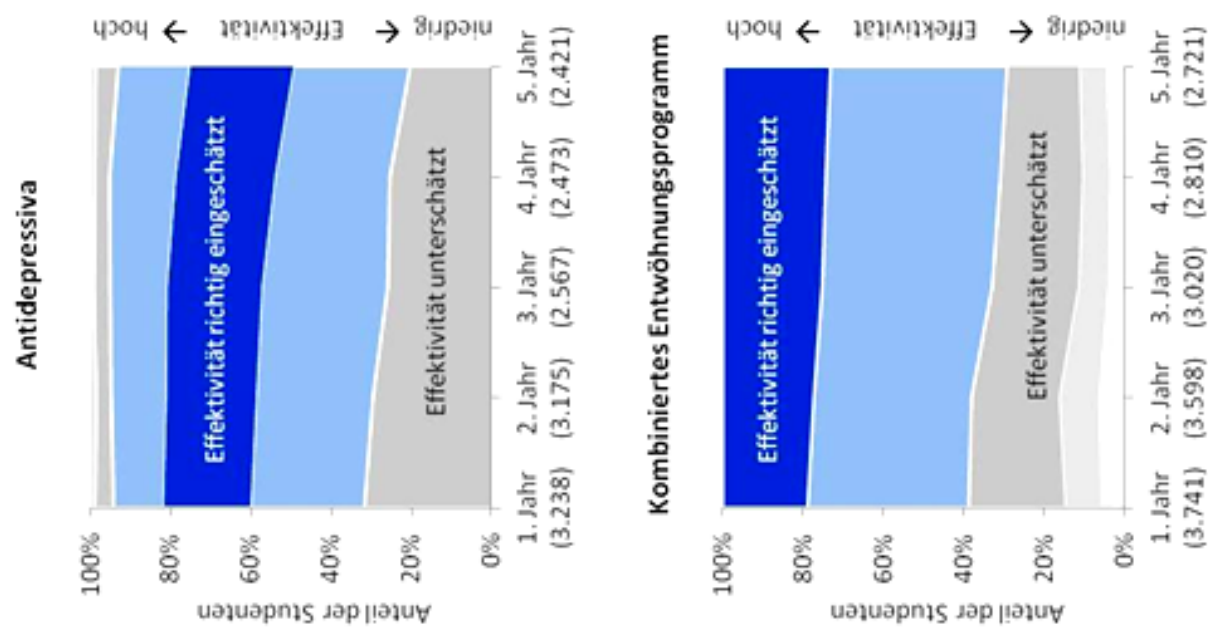

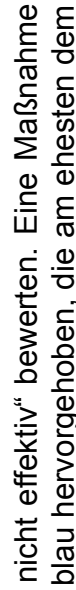

을

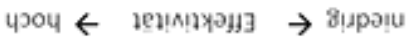
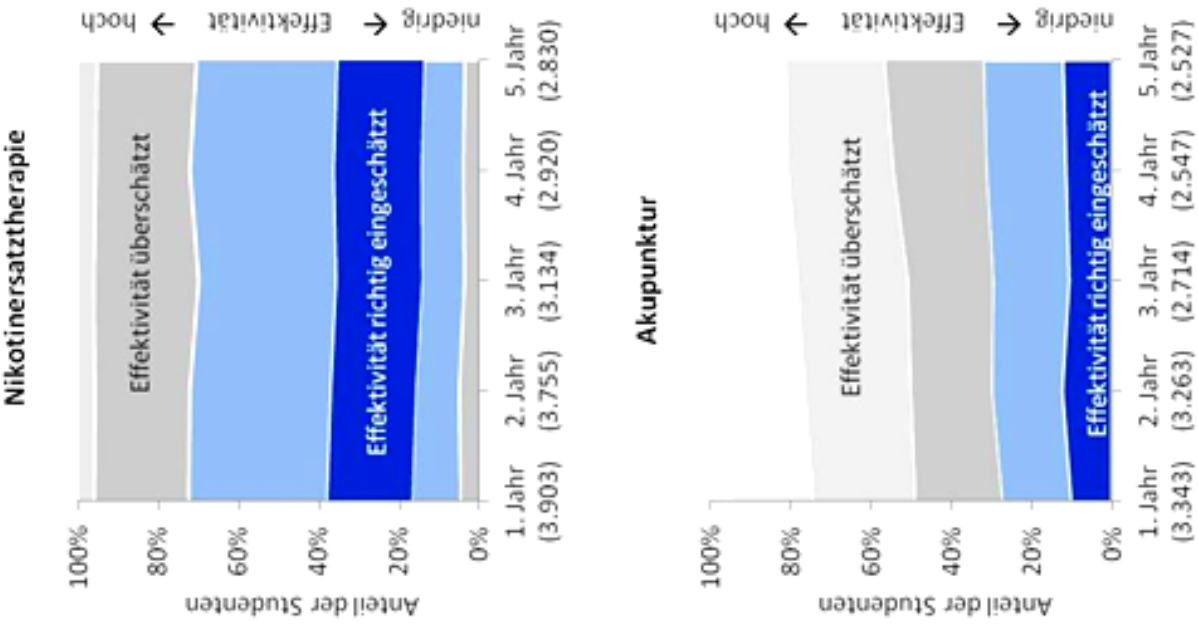

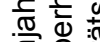

远:

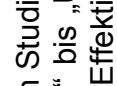

要这

$\frac{\text { 产 }}{\frac{3}{2}}$

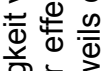

을

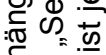

安 号

$\subseteq \frac{\pi}{0}$

बे के

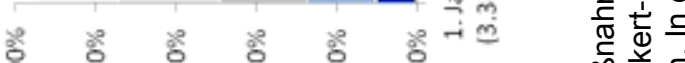

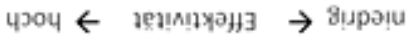
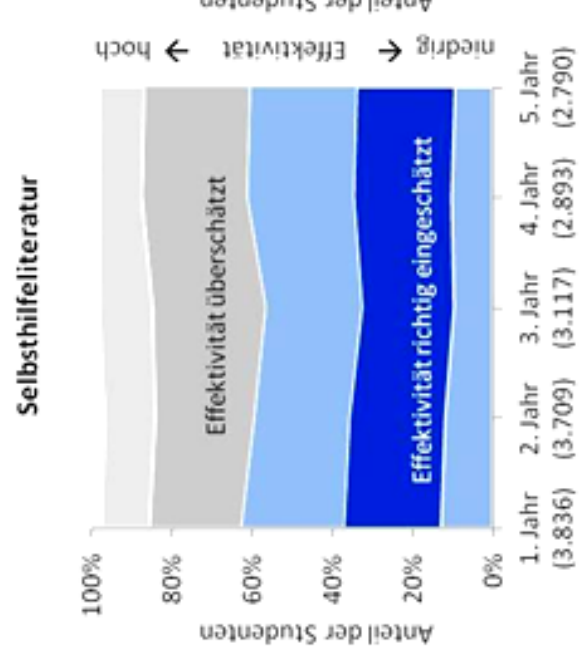

)

E 농

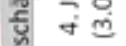

동 홍

का

竧忘专

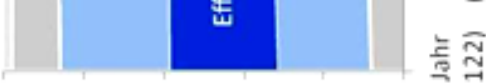

今े

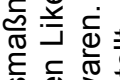

के

일

蛋 离

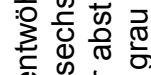

这市 离

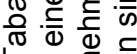

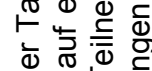

定

ब

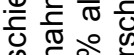

कू.

范

敋

迹

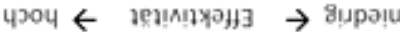
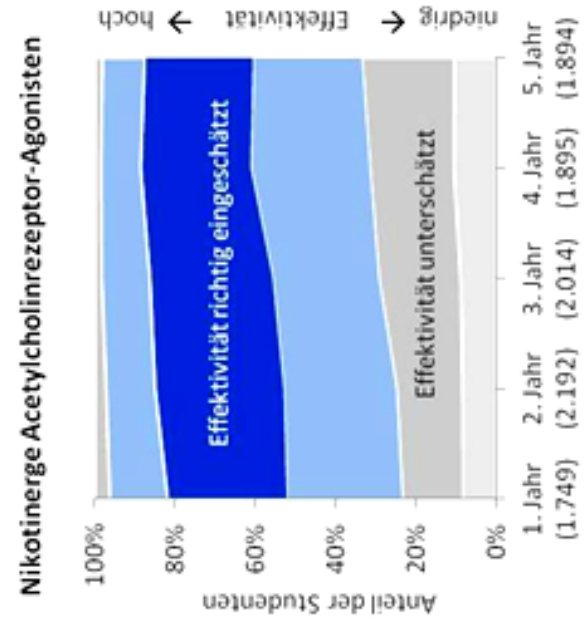

势

这

일을

:

空

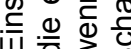

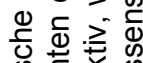

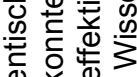

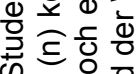

क ब

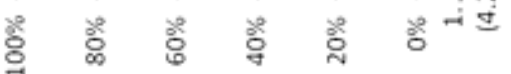

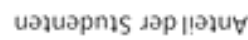

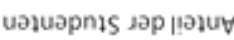

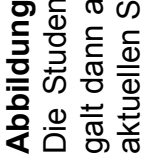



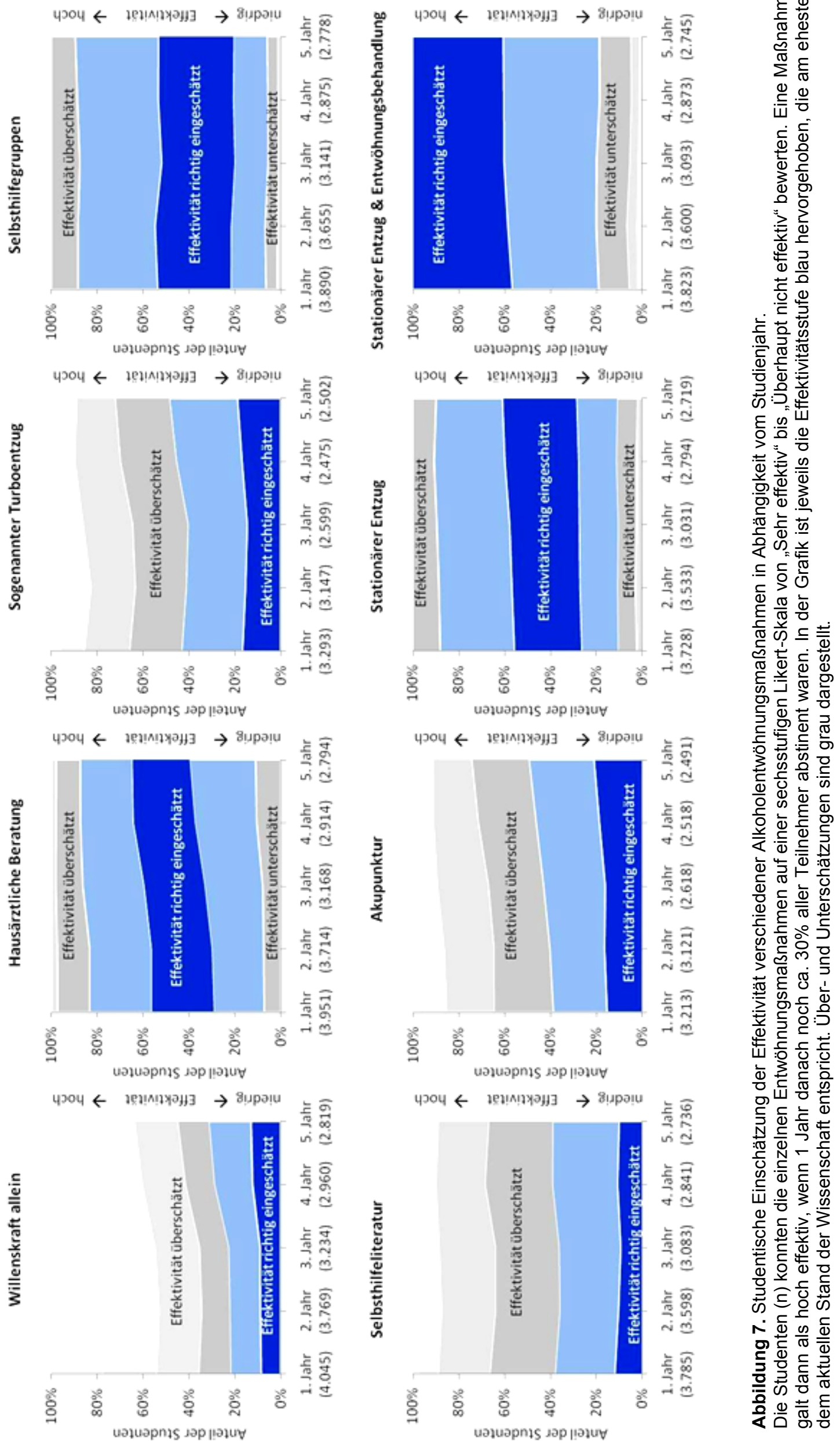


\begin{tabular}{lccccc} 
& $\begin{array}{c}\text { 1. Jahr } \\
(\mathbf{n = 4 . 3 0 2 )})\end{array}$ & $\begin{array}{c}\text { 2. Jahr } \\
(\mathbf{n = 4 . 0 1 9 )}\end{array}$ & $\begin{array}{c}\text { 3. Jahr } \\
(\mathbf{n}=\mathbf{3 . 3 7 2})\end{array}$ & $\begin{array}{c}\text { 4. Jahr } \\
(\mathbf{n}=\mathbf{3 . 0 3 9 )})\end{array}$ & $\begin{array}{c}\text { 5. Jahr } \\
(\mathbf{n}=\mathbf{2 . 9 0 5})\end{array}$ \\
\cline { 2 - 6 } Keine & $(\%)$ & $(\%)$ & $(\%)$ & $(\%)$ & $(\%)$ \\
$\mathbf{1}$ & 73,4 & 75,1 & 78,3 & 74,3 & 67,1 \\
$\mathbf{2}$ & 4,8 & 3,8 & 4,1 & 6,1 & 8,0 \\
$\mathbf{3}$ & 7,2 & 6,4 & 5,6 & 7,0 & 9,0 \\
$\mathbf{4}$ & 9,0 & 8,6 & 7,4 & 7,4 & 9,1 \\
Alle & 4,0 & 4,2 & 3,1 & 3,2 & 3,6 \\
\hline
\end{tabular}

Tabelle 14. Antworten auf die Frage: „Wie viele der 5 üblichen Maßnahmen in der Tabakentwöhnung, die als „5A“ bekannt sind, können Sie explizit benennen?“

\begin{tabular}{lccccc} 
& $\begin{array}{c}\text { 1. Jahr } \\
(\mathbf{n}=\mathbf{4 . 2 5 3})\end{array}$ & $\begin{array}{c}\text { 2. Jahr } \\
(\mathbf{n}=\mathbf{3 . 9 6 7 )})\end{array}$ & $\begin{array}{c}\text { 3. Jahr } \\
(\mathbf{n}=\mathbf{3 . 3 . 6 2})\end{array}$ & $\begin{array}{c}\text { 4. Jahr } \\
(\mathbf{n}=\mathbf{3 . 0 1 6})\end{array}$ & $\begin{array}{c}\text { 5. Jahr } \\
(\mathbf{n}=\mathbf{2 . 8 7 3})\end{array}$ \\
\cline { 2 - 7 } & $(\%)$ & $(\%)$ & $(\%)$ & $(\%)$ & $(\%)$ \\
\hline $\mathbf{1}$ & 83,5 & 83,0 & 86,7 & 86,3 & 78,3 \\
$\mathbf{2}$ & 2,9 & 2,2 & 2,6 & 3,0 & 3,7 \\
$\mathbf{3}$ & 3,7 & 3,9 & 2,8 & 3,5 & 5,7 \\
$\mathbf{4}$ & 4,4 & 4,8 & 3,7 & 4,0 & 5,4 \\
$\mathbf{5}$ & 3,0 & 3,3 & 2,5 & 1,8 & 3,5 \\
Alle & 1,2 & 1,0 & 0,8 & 0,6 & 0,9 \\
\hline
\end{tabular}

Tabelle 15. Antworten auf die Frage: „Wie viele der sechs Maßnahmen in der Beratung alkoholabhängiger Patienten, deren Anfangsbuchstaben in dem Akronym „FRAMES“ enthalten sind, können Sie explizit benennen?"

\subsubsection{Praxisverhalten und Beratungskompetenz}

Nahezu 90\% aller Studenten mit regelmäßigem Patientenkontakt gaben an, den Raucherstatus ihrer Patienten bei jeder Anamneseerhebung abzufragen; lediglich 60,9\% sprachen auch eine Rauchstoppempfehlung aus. Bei Betrachtung der Studenten in den Studienjahren 3 - 5 (siehe Tabelle 16) zeigte sich, dass der Anteil derjenigen, die angaben, den Rauchstatus ihrer Patienten zu dokumentieren, im letzten Studienjahr nicht größer war als zu Beginn des klinischen Abschnitts der Ausbildung (91,2\% vs. 91,8\%; $\chi^{2}$ : $p=$ 0,873). Im Gegensatz dazu war der Anteil der Studenten, der angab, eine Rauchstopp- 
empfehlung auszusprechen, im letzten Studienjahr niedriger als im dritten Studienjahr $\left(51,9 \%\right.$ vs. $\left.61,8 \% ; \chi^{2}: p<0.001\right)$.

Obwohl über die Hälfte aller Studenten angab, jedem rauchenden Patienten einen Rauchstopp zu empfehlen, fühlten sich insgesamt nur wenige Studenten kompetent genug, einen Raucher, der mit dem Rauchen aufhören mochte, entsprechend zu beraten (siehe Abbildung 8). Es existiert zwar eine schwache Korrelation zwischen studentischen Angaben und Ausbildungsstand (Spearman's rho $=0,15 ; \mathrm{p}<0,001$ ), dennoch betrug im letzten Studienjahr der Anteil jener Studenten, der seine Beratungskompetenz als hoch einstufte, noch nicht einmal $3 \%$.

3. Jahr

4. Jahr

5. Jahr

p-Wert

\begin{tabular}{lccccccc}
\hline & $(\mathrm{n})$ & $(\%)$ & $(\mathrm{n})$ & $(\%)$ & $(\mathrm{n})$ & $(\%)$ & $\left(\chi^{2}\right)$ \\
\cline { 2 - 8 } Jokumentation des Rauchstatus & 2.028 & 91,8 & 2.459 & 91,4 & 2.495 & 91,2 & 0,873 \\
Rauchstoppempfehlung & 1.422 & 61,8 & 1.435 & 54,3 & 1.394 & 51,9 & $<0,001$ \\
\hline
\end{tabular}

Tabelle 16. Anteil der Studenten ( $n$ ) mit regelmäßigem Patientenkontakt, die angaben, a) den Rauchstatus ihrer Patienten zu erfragen ( $\left.n_{\text {gesamt }}=7.635\right)$ und $b$ ) einen Rauchstopp zu empfehlen $\left(n_{\text {gesamt }}=7.628\right)$. Der $x^{2}$-Test wurde über alle Studienjahre hinweg berechnet.

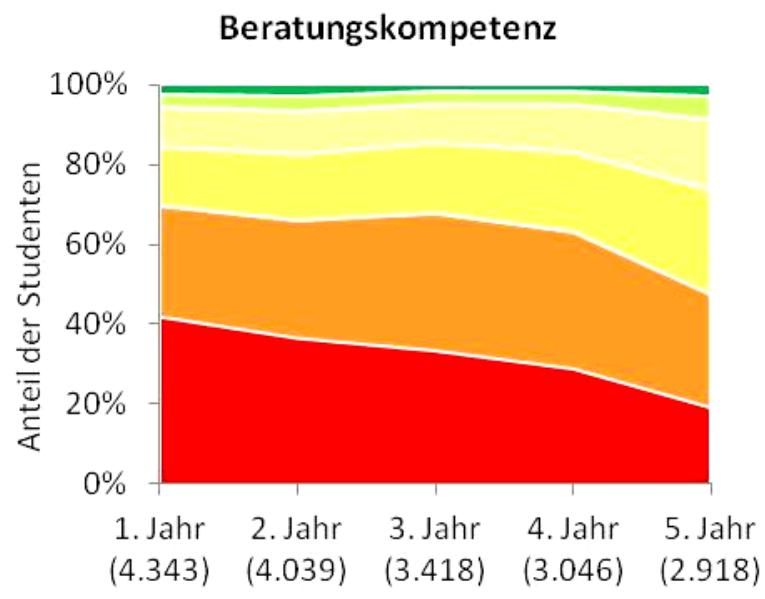

Abbildung 8. Studentische Selbsteinschätzung hinsichtlich der Kompetenz in der Raucherberatung. Die Studenten konnten die Aussage „Ich fühle mich kompetent, einen Raucher, der mit dem Rauchen aufhören möchte, zu beraten“ auf einer sechsstufigen Likert-Skala von „Trifft voll zu“ (grün) bis „Trifft überhaupt nicht zu“ (rot) bewerten. 


\subsubsection{Lehrsituation und Verbesserungswünsche}

Im letzten Studienjahr gaben knapp zwei Drittel der Studenten (64,6\%) an, während ihres Studiums theoretischen Unterricht zur Tabakabhängigkeit und deren Behandlungsmöglichkeiten erhalten zu haben. Von praktischem Unterricht berichteten hingegen nur $5 \%$ (siehe Tabelle 17).

Lediglich ein geringer Prozentsatz der Studenten sprach sich für eine Intensivierung der Lehre von arterieller Hypertonie und Diabetes mellitus aus (siehe Abbildung 9). Mit insgesamt 5\% war der Anteil der Fürsprecher im letzten Studienjahr niedriger als im ersten (20\%). Konträr hierzu war der Anteil der Studenten, der eine Intensivierung der Lehre von Tabak- und Alkoholabhängigkeit forderte, gegen Ende des Studiums am größten. Am deutlichsten wurde der Unterschied in den letzten beiden Studienjahren, in denen sich weit mehr als die Hälfte der Studenten für eine stärkere curriculare Repräsentation dieser Erkrankungen aussprach.

\begin{tabular}{lccccc} 
& 1. Jahr & 2. Jahr & 3. Jahr & 4. Jahr & 5. Jahr \\
\hline \multirow{2}{*}{ Theorie } & $(\%)$ & $(\%)$ & $(\%)$ & $(\%)$ & $(\%)$ \\
\cline { 2 - 6 } Praxis & 15.6 & 27.9 & 34.2 & 50.4 & 64.6
\end{tabular}

Tabelle 17. Anteil der Studenten, die nach eigenen Angaben während des Studiums theoretischen und/oder praktischen Unterricht zur Tabakentwöhnung erhalten haben.

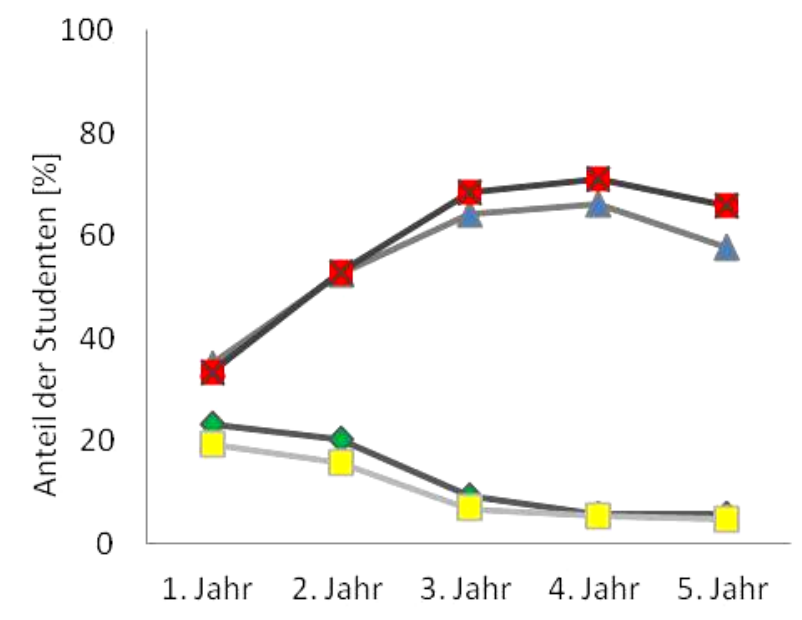

Abbildung 9. Anteil der Studenten, die sich eine intensivere Lehre für arterielle Hypertonie (【】), Diabetes mellitus $(\square)$, Tabak- $(\square)$ und Alkoholabhängigkeit $(\searrow)$ wünschten. 
Die nun folgenden Subgruppenanalysen beziehen sich jeweils nur auf Studierende des letzten Studienjahres.

\subsection{Unterschiede zwischen Rauchern und Nichtrauchern}

\subsubsection{Entwöhnungsmaßnahmen}

Tabelle 18 zeigt den Anteil der Raucher und Nichtraucher im letzten Studienjahr, die die Effektivität der aufgeführten Tabak- und Alkoholentwöhnungsmaßnahmen richtig eingeschätzt haben. Insgesamt konnten nur sehr wenige Studenten die Effektivität der einzelnen Entwöhnungsmaßnahmen richtig bewerten; im besten Fall („Stationärer Entzug und Entwöhnung“) waren es 42,5\%. Am schlechtesten wurde das Item „Willenskraft allein“ in der Tabakentwöhnung eingeschätzt; dessen Wirksamkeit konnte lediglich von 4,8\% der Raucher und 6,3\% der Nichtraucher richtig eingestuft werden. In den meisten Fällen war der Anteil der Nichtraucher, der die Maßnahme richtig einschätzte, etwas höher als der der Raucher. Lediglich in einem Fall („Kombiniertes Entwöhnungsprogramm“) war der Unterschied zwischen den Gruppen jedoch signifikant (28,5\% vs. $\left.21,7 \% ; \chi^{2}: p=0,002\right)$. Im Durchschnitt wurden die Maßnahmen zur Alkoholentwöhnung (Raucher: 23,1\%, Nichtraucher: $24,3 \%$ ) etwas besser eingeschätzt, als die Maßnahmen zur Tabakentwöhnung (Raucher: 19,8\%, Nichtraucher: 22,1\%).

\subsubsection{Komorbidität und Behandlung ausgewählter Erkrankungen}

Knapp 90\% der Studenten (Raucher und Nichtraucher) im letzten Studienjahr gaben an, die Folgeerkrankungen aller vier Erkrankungen zu kennen (siehe Tabelle 19). Ein ähnlich hoher Prozentsatz berichtete zu wissen, wie die arterielle Hypertonie und der Diabetes mellitus behandelt werden, wohingegen weniger als ein Drittel der Raucher und Nichtraucher angab, die Behandlungsoptionen von Alkohol- und Tabakabhängigkeit zu kennen. Signifikante Unterschiede zwischen den beiden Gruppen gab es lediglich für die Tabakabhängigkeit (Folgeerkrankungen: $88,1 \%$ vs. $79,8 \%, \chi^{2}$ : $p<0,001$; Behandlungsoptionen: $25,7 \%$ vs. $\left.21,3 \%, \chi^{2}: p=0,028\right)$.

\subsubsection{Praxisverhalten und Beratungskompetenz}

Gegen Ende des Studiums gaben etwa 90\% der Raucher und Nichtraucher an, den Raucherstatus ihrer Patienten regelmäßig zu dokumentieren. Ein etwas geringerer Anteil beider Gruppen (55\% der Raucher und 51,1\% der Nichtraucher) berichtete, ihren Patienten 
auch regelmäßig zu empfehlen, mit dem Rauchen aufzuhören. Für beide Variablen ließ sich kein signifikanter Unterschied zwischen den Gruppen feststellen.

Kompetent genug, um eine adäquate Raucherberatung durchzuführen, fühlten sich etwas mehr Raucher $(11,1 \%$ vs. $8,1 \% ; p=0,023)$ als Nichtraucher.

\begin{tabular}{lcc} 
& Raucher & Nichtraucher \\
\hline TABAKENTWÖHNUNG & $(\%)$ & $(\%)$ \\
\cline { 2 - 3 } Willenskraft allein & 4,8 & 6,3 \\
Hausärztliche Beratung & 26,9 & 28,4 \\
Nikotin - Ersatz - Therapie & 22,9 & 21,6 \\
Antidepressiva & 22,5 & 26,5 \\
Nikotinerge AchR-Agonisten* & 24,0 & 27,9 \\
Selbsthilfeliteratur & 22,2 & 25,2 \\
Akupunktur & 13,3 & 12,0 \\
Kombiniertes Entwöhnungsprogramm & 21,7 & $28,5 \S$ \\
ALKOHOLENTWöHNUNG & & \\
Willenskraft allein & 11,4 & 13,1 \\
Hausärztliche Beratung & 23,7 & 25,5 \\
Turbo - Entzug & 17,5 & 19,1 \\
Selbsthilfegruppen & 31,2 & 33,2 \\
Selbsthilfeliteratur & 10,3 & 10,2 \\
Akupunktur & 19,0 & 21,4 \\
Stationärer Entzug & 29,2 & 32,8 \\
Stationärer Entzug \& Entwöhnung & 42,5 & 38,9 \\
\hline
\end{tabular}

Tabelle 18. Anteil der Raucher/Nichtraucher im letzten Studienjahr, die die Effektivität der einzelnen Tabak- und Alkoholentwöhnungsmaßnahmen richtig eingeschätzt haben.

* Nikotinerge Acetylcholinrezeptor-Agonisten

§ Signifikanter Unterschied zwischen Rauchern und Nichtrauchern. Signifikanzniveau von $p<0,05$. 


\begin{tabular}{lcc} 
& Raucher & Nichtraucher \\
\hline FOLGEERKRANKUNGEN & $(\%)$ & $(\%)$ \\
\cline { 2 - 3 } Arterielle Hypertonie & 93,1 & $95,5 \S$ \\
Diabetes mellitus & 93,5 & $96,3 \S$ \\
Alkoholabhängigkeit & 89,7 & 87,3 \\
Tabakabhängigkeit & 88,1 & $79,8 \S$ \\
BEHANDLUNGSOPTIONEN & & \\
Arterielle Hypertonie & 85,6 & 87,4 \\
Diabetes mellitus & 87,1 & 87,7 \\
Alkoholabhängigkeit & 29,8 & 26,4 \\
Tabakabhängigkeit & 25,7 & $21,3 \S$ \\
\hline
\end{tabular}

Tabelle 19. Anteil der Raucher/Nichtraucher im letzten Studienjahr, die angaben, die Folgeerkrankungen und Behandlungsoptionen der einzelnen Erkrankungen zu kennen.

§ Signifikanter Unterschied zwischen Rauchern und Nichtrauchern. Signifikanzniveau von $p<0,05$.

\subsection{Geschlechtsspezifische Unterschiede}

\subsubsection{Entwöhnungsmaßnahmen}

Tabelle 20 zeigt den Anteil der Männer und Frauen im letzten Studienjahr, die die Effektivität der aufgeführten Tabak- und Alkoholentwöhnungsmaßnahmen richtig eingeschätzt haben. Unabhängig vom Geschlecht konnten insgesamt nur sehr wenige Studenten die Effektivität der einzelnen Entwöhnungsmaßnahmen richtig bewerten; für einige Maßnahmen fanden sich jedoch geschlechtsspezifische, signifikante Unterschiede.

Während der Wirkungsgrad von „Willenskraft allein“ in der Tabakentwöhnung $(8,1 \%$ vs. $\left.4,7 \% ; \chi^{2}: p<0,001\right)$ und „Akupunktur“ in der Tabak- und Alkoholentwöhnung $(17,2 \%$ vs. $9,7 \% ; \chi^{2}: p<0,001$ und $24 \%$ vs. $\left.19,4 \% ; \chi^{2}: p=0,008\right)$ von Männern häufiger richtig als von Frauen eingeschätzt wurde, stuften signifikant mehr Frauen die Effektivität verhaltenstherapeutischer Maßnahmen wie „Kombiniertes Entwöhnungsprogramm“ und „Stationärer Entzug \& Entwöhnung" richtig ein (30,1\% vs. 21,8\%; $\chi^{2}$ : $p<0,001$ und $42,2 \%$ vs. 34,5\%; $\left.\chi^{2}: p<0,001\right)$. Im Durchschnitt wurden die Maßnahmen zur Alkoholentwöhnung (Männer: 24,2\%, Frauen: 21,4\%) etwas besser eingeschätzt, als die Maßnahmen zur Tabakentwöhnung (Männer: 22,0\%, Frauen: 23,8\%). 


\begin{tabular}{lcc} 
& Männer & Frauen \\
\hline TABAKENTWÖHNUNG & $(\%)$ & $(\%)$ \\
\cline { 2 - 3 } Willenskraft allein & 8,1 & $4,7 \S$ \\
Hausärztliche Beratung & 26,1 & 29,6 \\
Nikotin -Ersatz - Therapie & 24,0 & $20,3 \S$ \\
Antidepressiva & 27,6 & 24,1 \\
Nikotinerge AchR-Agonisten* & 25,0 & 28,3 \\
Selbsthilfeliteratur & 26,0 & 24,1 \\
Akupunktur & 17,2 & $9,7 \S$ \\
Kombiniertes Entwöhnungsprogramm & 21,8 & $30,1 \S$ \\
ALKOHOLENTWÖHNUNG & & \\
Willenskraft allein & 13,5 & 12,2 \\
Hausärztliche Beratung & 25,6 & 24,9 \\
Turbo - Entzug & 18,7 & 18,6 \\
Selbsthilfegruppen & 34,5 & 31,3 \\
Selbsthilfeliteratur & 11,1 & 9,5 \\
Akupunktur & 24,0 & $19,4 \S$ \\
Stationärer Entzug & 31,5 & 31,9 \\
Stationärer Entzug \& Entwöhnung & 34,5 & $42,2 \S$ \\
\hline
\end{tabular}

Tabelle 20. Anteil der Männer/Frauen im letzten Studienjahr, die die Effektivität der einzelnen Tabak- und Alkoholentwöhnungsmaßnahmen richtig eingeschätzt haben.

* Nikotinerge Acetylcholinrezeptor-Agonisten

$\S$ Signifikanter Unterschied zwischen Männern und Frauen. Signifikanzniveau von $p<0,05$.

\subsubsection{Komorbidität und Behandlung ausgewählter Erkrankungen}

Unabhängig vom Geschlecht gab die Mehrheit der Studenten im letzten Studienjahr an, die Folgeerkrankungen von arterieller Hypertonie, Diabetes mellitus, Alkohol- und Tabakabhängigkeit zu kennen (siehe Tabelle 21). Ein ähnlich hoher Prozentsatz berichtete zu wissen, wie die arterielle Hypertonie und der Diabetes mellitus behandelt werden, wohingegen weniger als ein Drittel der Männer und Frauen angab, die Behandlungsoptionen von Alkohol- und Tabakabhängigkeit zu kennen. Frauen berichteten im Falle der Folgeerkrankungen von arterieller Hypertonie und Diabetes mellitus und der Behandlungsoptionen des Diabetes mellitus signifikant häufiger als Männer, diese zu kennen. 


\begin{tabular}{lcc} 
& Männer & Frauen \\
\hline FOLGEERKRANKUNGEN & $(\%)$ & $(\%)$ \\
\cline { 2 - 3 } Arterielle Hypertonie & 93,8 & $95,6 \S$ \\
Diabetes mellitus & 94,5 & $96,6 \S$ \\
Alkoholabhängigkeit & 87,6 & 88,0 \\
Tabakabhängigkeit & 79,9 & 82,9 \\
BEHANDLUNGSOPTIONEN & & \\
Arterielle Hypertonie & 86,4 & 87,6 \\
Diabetes mellitus & 86,0 & $89,1 \S$ \\
Alkoholabhängigkeit & 27,2 & 26,9 \\
Tabakabhängigkeit & 22,9 & 22,2 \\
\hline
\end{tabular}

Tabelle 21. Anteil der Männer/Frauen im letzten Studienjahr, die angaben, die Folgeerkrankungen und Behandlungsoptionen der einzelnen Erkrankungen zu kennen.

§ Signifikanter Unterschied zwischen Männern und Frauen. Signifikanzniveau von $p<0,05$.

\subsubsection{Praxisverhalten und Beratungskompetenz}

Etwa 90\% der Frauen und Männer berichteten, den Rauchstatus ihrer Patienten zu dokumentieren (91,7\% vs. 90,1\%). Der Anteil derer, die angaben, eine Rauchstoppempfehlung auszusprechen, betrug in beiden Gruppen nur etwa 50\% (50,3\% der Frauen vs. $53,7 \%$ der Männer). Die Unterschiede zwischen den Gruppen waren nicht signifikant. Der Anteil der Männer und Frauen, der sich kompetent genug fühlte, um eine adäquate Raucherberatung durchzuführen, war im letzten Studienjahr ähnlich gering $(8,2 \%$ vs. $9,6 \%$; $p=0,230)$.

\subsection{Unterschiede zwischen Regel- und Modellstudiengang}

\subsubsection{Entwöhnungsmaßnahmen}

Tabelle 22 zeigt den Anteil der Studenten aus Regel- und Modellstudiengängen im letzten Studienjahr, die die Effektivität der aufgeführten Tabak- und Alkoholentwöhnungsmaßnahmen richtig eingeschätzt haben. Signifikante Unterschiede gab es lediglich für das Item „Selbsthilfeliteratur“ in der Tabakentwöhnung (25\% vs. 19,1\%; $\left.\chi^{2}: p=0,028\right)$ und das Item „Akupunktur“ in der Alkoholentwöhnung (21,2\% vs. 19,4\%; $\left.\chi^{2}: p=0,008\right)$. Die Effekti- 
vität dieser Maßnahmen wurde von Studenten aus Modellstudiengängen häufiger richtig eingeschätzt.

\begin{tabular}{lcc} 
& RSM & MSM \\
\hline TABAKENTWÖHNUNG & $(\%)$ & $(\%)$ \\
\cline { 2 - 3 } Willenskraft allein & 6,4 & 3,6 \\
Hausärztliche Beratung & 28,8 & 24,9 \\
Nikotin - Ersatz - Therapie & 21,7 & 22,1 \\
Antidepressiva & 25,3 & 30,5 \\
Nikotinerge AchR-Agonisten* & 26,9 & 25,5 \\
Selbsthilfeliteratur & 25,0 & $19,1 \S$ \\
Akupunktur & 12,3 & 12,4 \\
Kombiniertes Entwöhnungsprogramm & 27,0 & 24,6 \\
ALKOHOLENTWöHNUNG & & \\
Willenskraft allein & 13,1 & 10,7 \\
Hausärztliche Beratung & 25,0 & 25,5 \\
Turbo - Entzug & 18,9 & 16,6 \\
Selbsthilfegruppen & 33,1 & 30,4 \\
Selbsthilfeliteratur & 10,3 & 8,2 \\
Akupunktur & 21,2 & $19,4 \S$ \\
Stationärer Entzug & 32,1 & 32,6 \\
Stationärer Entzug \& Entwöhnung & 38,9 & 42,2 \\
\hline
\end{tabular}

Tabelle 22. Anteil der Studenten aus Regel- (RSM) und Modellstudiengängen (MSM) im letzten Studienjahr, die die Effektivität der einzelnen Tabak- und Alkoholentwöhnungsmaßnahmen richtig eingeschätzt haben. * Nikotinerge Acetylcholinrezeptor-Agonisten $\S$ Signifikanter Unterschied zwischen Studenten des Regelstudienganges und Studenten des Modellstudienganges. Signifikanzniveau von $p<0,05$.

\subsubsection{Komorbidität und Behandlung ausgewählter Erkrankungen}

Die Ergebnisse dieser Subgruppenanalyse präsentieren sich in ähnlicher Weise wie unter 3.3.2 und 3.4.2. Die Mehrheit der Studenten im letzten Studienjahr - unabhängig von der Art des Studienganges - gab an, die Folgeerkrankungen von arterieller Hypertonie, Diabetes mellitus, Alkohol- und Tabakabhängigkeit zu kennen (siehe Tabelle 19). Ein ähnlich hoher Prozentsatz berichtete zu wissen, wie die arterielle Hypertonie und der Diabetes 
mellitus behandelt werden, wohingegen weniger als ein Drittel der Studenten aus Regelund Modellstudiengängen angab, die Behandlungsoptionen von Alkohol- und Tabakabhängigkeit zu kennen. Für die geringfügigen Unterschiede zwischen den Gruppen ließ sich keine statistische Signifikanz nachweisen (siehe Tabelle 23).

\begin{tabular}{lcc} 
& RSM & MSM \\
\hline FOLGEERKRANKUNGEN & $(\%)$ & $(\%)$ \\
\cline { 2 - 3 } Arterielle Hypertonie & 94,9 & 94,2 \\
Diabetes mellitus & 95,6 & 97,1 \\
Alkoholabhängigkeit & 87,9 & 87,2 \\
Tabakabhängigkeit & 81,4 & 83,7 \\
BEHANDLUNGSOPTIONEN & & \\
Arterielle Hypertonie & 87,2 & 85,1 \\
Diabetes mellitus & 87,6 & 89,3 \\
Alkoholabhängigkeit & 27,0 & 28,7 \\
Tabakabhängigkeit & 23,0 & 18,3 \\
\hline
\end{tabular}

Tabelle 23. Anteil der Studenten aus Regel- (RSM) und Modellstudiengängen (MSM) im letzten Studienjahr, die angaben, die Folgeerkrankungen und Behandlungsoptionen der einzelnen Erkrankungen zu kennen.

\subsubsection{Praxisverhalten und Beratungskompetenz}

Der Anteil derer, die berichteten, den Rauchstatus ihrer Patienten regelmäßig zu dokumentieren (91\% des Regelstudiengangs vs. 92,6\% des Modellstudienganges) und eine Rauchstoppempfehlung auszusprechen (51,6\% des Regelstudiengangs vs. $54,5 \%$ des Modellstudiengangs), war in beiden Gruppen ähnlich groß.

Studenten aus Modellstudiengängen fühlten sich im letzten Studienjahr etwas häufiger als Studenten aus Regelstudiengängen kompetent genug, um eine adäquate Raucherberatung durchzuführen $(11,9 \%$ vs. $8,4 \% ; p=0,035)$. 


\section{Diskussion}

\subsection{Zusammenfassung der Ergebnisse}

Diabetes mellitus und arterielle Hypertonie wurden mit durchschnittlich 315 beziehungsweise 275 Minuten deutlich umfangreicher unterrichtet als Tabak- und Alkoholabhängigkeit (135 und 180 Minuten). Vorlesungen und Seminare wurden an den meisten Fakultäten als Unterrichtsmethode präferiert.

An der Studierendenbefragung beteiligten sich insgesamt 19.528 Studenten von 27 medizinischen Hochschulen. Unabhängig vom Ausbildungsfortschritt bezeichnete sich durchschnittlich jeder fünfte Student als gelegentlicher oder regelmäßiger Raucher.

Gegen Ende des Studiums schätzten die Studenten ihr Wissen zu Komorbiditäten der vier Erkrankungen ausreichend hoch ein. Ähnlich verhielt es sich für die Behandlungsmöglichkeiten von arterieller Hypertonie und Diabetes mellitus, wohingegen weniger als die Hälfte aller Studenten wusste, wie die Tabak- und Alkoholabhängigkeit behandelt werden. Entsprechende Beratungsstrategien wie die 5A und FRAMES waren nur wenigen Studenten bekannt; effektive medikamentöse und verhaltenstherapeutische Entwöhnungsmaßnahmen wurden von der Mehrheit der Studenten unterschätzt.

Im letzten Studienjahr berichteten nur etwa $50 \%$ der Studenten, gegenüber ihren Patienten eine Rauchstopp-Empfehlung auszusprechen. Der Anteil an Studenten, die sich an praktischen Unterricht zum Umgang mit Rauchern und zum Thema Tabakentwöhnung erinnerten, war mit 5\% sehr gering. Entsprechend hielt sich nur jeder zwanzigste Student für kompetent genug, um eine adäquate Raucherberatung durchzuführen. Weit mehr als die Hälfte der Studenten sprach sich gegen Ende des Studiums für eine Intensivierung der Lehre zu den Themen „Tabak- und Alkoholabhängigkeit“ aus.

\subsection{Diskussion der Ergebnisse}

\subsubsection{Curriculumanalyse}

Seit den Neunziger Jahren hat sich die Anzahl von Universitäten, die einen spezifischen Tabak-Kurs anbieten, weltweit verdoppelt (Richmond und Kehoe 1997, Richmond et al. 2009). Zeitgleich wurde für eine verstärkte Verankerung/Repräsentation alkoholbezogener Inhalte in medizinischen Curricula eingetreten (Fleming et al. 1994, Roche 1992). Die Ergebnisse dieser deutschlandweiten Untersuchung zeigen, dass knapp zwanzig Jahre später hierzulande immer noch starke curriculare Defizite auf den Gebieten der Tabakund Alkoholabhängigkeit bestehen. Zudem bestätigt sich die Hypothese, dass chronische 
Suchterkrankungen nicht mit der gleichen Intensität unterrichtet werden wie „klassische“ internistische Erkrankungen. Warum genau dieser Unterschied existiert, bleibt unklar. Ein möglicher Erklärungsansatz kann darin gesehen werden, dass die Unterrichtsgestaltung zum Thema Abhängigkeitserkrankungen, aufgrund der Natur der Erkrankungen, sehr viel komplexer und daher unbeliebter ist als die zu arterieller Hypertonie und Diabetes mellitus. Die Ergebnisse der hier vorgelegten Studie bestätigen auch, dass Vorlesungen immer noch zu den am häufigsten eingesetzten Lehrmethoden zählen, während praxisbezogene Kurse zur Diagnostik und Therapie der Tabak- und Alkoholabhängigkeit wesentlich seltener in medizinische Curricula integriert sind. Dabei konnte bereits mehrfach gezeigt werden, dass praxisorientierte und interaktive Lehrformate, wie Rollenspiele oder die Arbeit mit Simulationspatienten, zur Verbesserung von Kommunikations- und Beratungsfähigkeiten beitragen (Roche et al. 1996, Humair und Cornuz 2003, Kosowicz et al. 2007, Proude et al. 2008).

\subsubsection{Demographie und Rauchverhalten}

Ein Raucheranteil von $20 \%$ spricht für eine Abnahme der Rauchprävalenz unter Medizinstudenten in Deutschland. Erhebungen Ende der Achtziger Jahre wiesen noch auf eine Raucherquote von etwa 40\% hin (von Troschke und Westenhoff 1989). Im Jahr 1992 betrug die Raucherquote unter Ulmer Medizinstudenten 17,6\% für Frauen und 29,2\% für Männer (Brenner und Scharrer 1996). Im globalen Vergleich nimmt Deutschland einen Platz im Mittelfeld ein. Besonders niedrig ist die Raucherprävalenz in den USA (3\%), während hingegen in Japan sechs von zehn Medizinstudenten rauchen (Smith und Leggat 2007). Die Vergleichbarkeit der Studien ist jedoch durch eine nicht immer einheitliche Definition des Terminus „Raucher" erschwert.

Der Anteil von Rauchern war trotz zunehmenden Wissens über die schädigenden Wirkungen des Rauchens im letzten Studienjahr so groß wie zu Beginn des Studiums. Ältere Studien hatten sogar über eine Zunahme der Raucherquote während des Studiums berichtet (Tessier et al. 1989, Richmond und Kehoe 1997). In der vorliegenden Untersuchung zeigte sich jedoch, dass die Bereitschaft, mit dem Rauchen aufzuhören, im letzten Studienjahr signifikant größer war als im ersten Studienjahr. Das Nebeneinander von Problembewusstsein und Umsetzungsschwierigkeiten betont einmal mehr, dass es sich beim Rauchen um ein chronisches Suchtverhalten handelt, das ohne professionelle Hilfe kaum aufgegeben werden kann.

Die Befürwortung des öffentlichen Rauchverbots war unter Medizinstudenten mit 83,4\% etwas größer als in der Allgemeinbevölkerung, wo sie Umfragen aus dem selben Jahr zufolge $73,4 \%$ betrug (Mons und Pötschke-Langer 2009). Insgesamt war der Anteil der 
Befürworter im letzten Studienjahr am größten, was ein im Laufe des Studiums stärker gewordenes Bewusstsein für die Gefahren des Rauchens und die zwingende Notwendigkeit dieser Maßnahme vermuten lässt.

\subsubsection{Entwöhnungsmaßnahmen und Beratungsstrategien}

Ohne professionelle Unterstützung gelingt es nur 2 - $5 \%$ aller Raucher, das Rauchen dauerhaft einzustellen (West et al. 2000). Vor diesem Hintergrund erschrickt die Tatsache, dass die Effektivität von „Willenskraft allein“ im Rahmen der Tabak- und Alkoholentwöhnung von den meisten Studenten überschätzt wird. Nicht zuletzt, wenn man bedenkt, welche Konsequenzen diese Einstellung auf späteres Handeln im ärztlichen Beruf hat. Sie zeigt darüber hinaus, dass Rauchen und Alkoholkonsum selbst von einem großen Teil zukünftiger Ärzte weniger als Suchterkrankung, sondern vielmehr als bewusste Lebensstilführung aufgefasst werden. Ärztliche Kurzinterventionen hingegen, deren Effektivität wissenschaftlich belegt ist (Fiore et al. 2008, Miller WR und Wilbourne 2002), wurden von knapp der Hälfte der Studenten beständig unterschätzt. Es ist wenig wahrscheinlich, dass zukünftige Ärzte der Beratung rauchender und alkoholabhängiger Patienten viel Aufmerksamkeit schenken, wenn sie von der Effektivität der Intervention nicht überzeugt sind (Vogt et al. 2006). Der Stellenwert von Antidepressiva (Bupropion), nikotinergen Acetylcholin-Rezeptor-Agonisten (Vareniclin) und eines kombinierten Entwöhnungsprogrammes in der Behandlung der Tabakabhängigkeit wurden ebenfalls unterschätzt, was dazu führen kann, dass nicht allen Patienten die Nutzung dieser Maßnahmen empfohlen wird. Die Tatsache, dass Akupunktur von so vielen Studenten für ein effektives Mittel sowohl in der Behandlung der Tabak- als auch der Alkoholabhängigkeit gehalten wurde, bedarf weiterer Klärung. Zumindest für die Tabakentwöhnung konnte bislang kein signifikanter Effekt gezeigt werden (White et al. 2011). Die Bedeutsamkeit eines stationären Entzuges mit anschließender Entwöhnung für alkoholabhängige Patienten wurde lediglich von $40 \%$ der Studenten erkannt. Dadurch wird auch verständlicher, warum nur 3\% aller alkoholabhängigen Patienten in entsprechende Suchtzentren überwiesen und dort therapiert werden (Mann 2002). Ein in diesem Zusammenhang oft diskutiertes Problem spielt neben fehlendem Fachwissen auch die unzureichende Vernetzung von bestehenden Hilfsprogrammen (Marcea et al. 2001).

\subsubsection{Komorbidität und Behandlung ausgewählter Erkrankungen}

Die unterschiedlich hohen Wissenseinschätzungen der Studierenden auf die Fragen zu Komorbiditäten und Behandlungsoptionen stützen die These, dass sich medizinische Ausbildung zu sehr auf Folgeerkrankungen und zu wenig auf Therapiekonzepte fokus- 
siert. Die festgestellte Diskrepanz zwischen den Einschätzungen zu Folgeerkrankungen und Behandlungsoptionen von arterieller Hypertonie / Diabetes mellitus und Tabak- / Alkoholabhängigkeit kann vor allem durch die weniger starke curriculare Verankerung und damit geringere Unterrichtsintensität der beiden letzten Erkrankungen erklärt werden (siehe 4.2.1). Am größten war der Unterschied zwischen den beiden Gruppen bei der Frage nach Behandlungsoptionen. Gerade hier finden sich ja auch theoretisch grundlegende Unterschiede. Während arterielle Hypertonie und Diabetes mellitus vor allem medikamentös kontrolliert werden können, kommen Tabak- und Alkoholentwöhnung ohne motivierende Gesprächsführung und Beratung nicht aus. Im Gegensatz zu Arzneimittellehre erfordert die Vermittlung solcher Kompetenzen aber zwingend praxisorientiertere Lehrmethoden als Vorlesungen (Levenkron et al. 1990). Eine Metaanalyse von Studien, die sich im Zeitraum von 1996 bis Juni 2002 mit Lehrmethoden zur Tabakentwöhnung beschäftigt haben, zeigt, dass interaktive Lehrmethoden wie Rollenspiele und / oder die Arbeit mit Simulationspatienten effektiver sind, als traditionelle Lehrformen allein (Spangler et al. 2002). Gerade diese werden jedoch von einer Vielzahl der Universitäten nicht angeboten (siehe 4.2.1).

Obwohl die Studenten ihre eigene Kompetenz hinsichtlich Folgeerkankungen und Behandlungsoptionen von arterieller Hypertonie und Diabetes mellitus deutlich besser einschätzen, existieren jedoch auch für diese beiden Erkrankungen deutliche Versorgungsmängel in Deutschland (Ott et al. 2009, Wolf-Maier et al. 2003). Ein möglicher Erklärungsansatz für diese Diskrepanz mag darin liegen, dass im Studium zwar wesentliche Grundlagen vermittelt werden, die endgültige Qualität der Versorgungssituation jedoch nicht nur von diesen Grundlagen allein abhängt. Als Einfluss-nehmende Variablen sind auch hier Zeit- und Vergütungsmangel zu diskutieren.

Durch das in England im Jahr 2004 eingeführte „Pay-for-Performance-System“, in dem das Erreichen bestimmter Qualitätsstandards im hausärztlichen Bereich extra vergütet wird, konnte zum Beispiel eine Steigerung der Versorgungsqualität nachgewiesen werden (Simpson et al. 2011). Die Möglichkeit einer Qualitätsverbesserung durch Vergütung ist jedoch nur dann möglich, wenn entsprechende Wissensgrundlagen, durch ein adäquates Studium vermittelt, existieren.

\subsubsection{Praxisverhalten und Beratungskompetenz}

Schon früh wurde postuliert, dass die geringe Dokumentations- und Behandlungshäufigkeit von Suchtkranken auf eine grundsätzlich negative und im Laufe des Studiums immer negativer werdende Einstellung gegenüber Abhängigkeitserkrankungen zurückzuführen ist (Fisher et al. 1975, Ritson 1991, Miller et al. 2001). Dazu würde auch der zunächst 
überraschende Befund passen, dass Studenten im letzten Studienjahr wesentlich seltener als zu Beginn des Studiums einen Rauchstopp empfehlen. Eine longitudinale Untersuchung von 286 Medizinstudenten aus Neuseeland konnte ähnliche Ergebnisse präsentieren (Cape et al. 2006). Obwohl der Anteil an Studenten mit adäquaten theoretischen Kenntnissen und entsprechender Kompetenz im Umgang mit alkohol- und / oder drogenabhängigen Patienten im Laufe des Studiums immer mehr zunahm (23,4\% im zweiten Studienjahr vs. $71,8 \%$ im sechsten Studienjahr), fühlten sich immer weniger Studenten in der Lage, solchen Patienten effektiv helfen zu können $(21 \%$ im zweiten Studienjahr vs. $51 \%$ im letzten Studienjahr).

Die regelmäßige Dokumentation des Rauchstatus wurde jedoch durchgehend von 90\% der Studierenden bejaht. Die Feststellung, dass rauchende Medizinstudenten / Ärzte mit ihren Patienten signifikant weniger häufig die Notwendigkeit eines Tabakverzichts thematisieren als Nichtraucher (Tessier et al. 1989, Tessier et al. 1992b), konnte in dieser Studie nicht getroffen werden.

Der Anteil an Medizinstudenten, die sich in der Lage fühlen, einen Raucher adäquat zu beraten, ist deutschlandweit insgesamt niedriger als in einer im Jahr 2009 untersuchten Stichprobe von Medizinstudenten in Göttingen (Raupach et al. 2009). Dieser Umstand ist besorgniserregend und nicht zuletzt für die schlechte Versorgungsqualität der Tabakabhängigkeit in Deutschland verantwortlich (Skoeries et al. 2010).

Die Tatsache, dass sich im letzten Studienjahr nicht mehr Studenten als zu Beginn für fähig hielten, eine Raucherberatung durchzuführen, lässt sich vielleicht dadurch erklären, dass mit zunehmend klinischer Erfahrung die besondere Herausforderung einer solchen Beratung von Studierenden erkannt wird.

\subsubsection{Lehrsituation und Verbesserungswünsche}

Das mangelnde Vertrauen in die eigenen Beratungsfähigkeiten verwundert nicht, wenn man den Anteil derer betrachtet, die überhaupt praktischen Unterricht zur Tabakentwöhnung erhalten haben. Selbst unter Berücksichtigung eines gewissen Recall-Bias war dieser Anteil sehr gering (5\%).

Die wohl umfangreichsten internationalen Daten hierzu liefert der „Global Health Professional Survey“, der von der World Health Organization (WHO), Centers for Disease Control and Prevention (CDC) und der Canadian Public Health Association (CPHC) entwickelt wurde. 
Im Rahmen dieses Surveys wurden Studierende und Auszubildende verschiedener Gesundheitsberufe im dritten Jahr an insgesamt 80 Studienzentren weltweit zu ihrer Ausbildung hinsichtlich Tabakentwöhnung befragt. Gleichwenn sich die Mehrzahl der Studenten eine entsprechende Ausbildung zur Tabakentwöhnung wünschte, konnte an 73 von 80 Studienzentren von nur weniger als $40 \%$ der Studenten davon berichten werden, einen solchen Unterricht erhalten zu haben (Warren et al. 2008).

In Südamerika werden Raucherberatungskurse nur in zwei von neun Ländern flächendeckend an den Universitäten angeboten, obwohl es mittlerweile in fünf der neun Länder Raucherentwöhnungsambulanzen gibt (Chatkin J und Chatkin G 2009).

Ein anderer Erklärungsansatz für das mangelnde Vertrauen in die eigenen Beratungsfähigkeiten mag der Glaube daran sein, dass Tabakentwöhnung nicht ärztliche Verantwortung, sondern eine Entscheidung des Patienten allein sei (Chatkin J und Chatkin G 2009). Hierbei werden jedoch die Rolle des Arztes als gesellschaftliches Vorbild und dessen Interventionsmöglichkeiten im Rahmen der Tabakentwöhnung völlig außer Acht gelassen.

Der Wunsch nach einer intensiveren Lehre von Tabak- und Alkoholabhängigkeit wurde von Studierenden schon vor Jahrzehnten geäußert (Tessier et al. 1992b). Im Jahr 2009 sprechen sich in Deutschland immer noch weit mehr als die Hälfte aller Studenten für eine verstärkte Beschäftigung mit diesen Themen aus. Die höhere Zustimmung in den Studienjahren 3 - 5 könnte darauf zurückzuführen sein, dass eine gesteigerte Auseinandersetzung mit dem klinischen Alltag den Studierenden auch immer mehr die Notwendigkeit einer intensiveren Lehre von Tabak- und Alkoholentwöhnungsmaßnahmen vor Augen führt.

\subsubsection{Subgruppenanalysen}

In den durchgeführten Subgruppenanalysen wurden nur wenige Gruppenunterschiede gefunden. So schätzten zum Beispiel Raucher ihr Wissen zu Behandlungsmöglichkeiten der Tabakabhängigkeit signifikant besser ein als Nichtraucher. Gründe hierfür mögen in einer privat tatsächlich verstärkten Auseinandersetzung mit der Thematik liegen oder aber auch dadurch zustande kommen, dass Raucher aus persönlicher Betroffenheit glauben am besten zu wissen, welche Entwöhnungsstrategien existieren und funktionieren.

Die Unterschiede zwischen Männern und Frauen, sowie Studenten des Regel- und Modellstudiengangs waren gering, Signifikanzen sind am ehesten auf die Größe der Stichprobe zurückzuführen. Ungeachtet dessen, ist die Konsequenz (geschlechtergetrenntes Studium) von wenig praktischer Relevanz. 


\subsection{Methodische Stärken und Schwächen}

Eine besondere Stärke der vorliegenden Arbeit war die Größe der befragten Stichprobe. Fast die Hälfte aller deutschen Medizinstudenten und drei Viertel aller medizinischen Fakultäten wurden in die Studie eingeschlossen; effektive Rücklaufquoten von $80-90 \%$ sprechen für eine angemessen hohe Repräsentativität der Ergebnisse. Diskutiert werden muss in diesem Zusammenhang jedoch ein möglicher durch Nichtbeteiligung einzelner Fakultäten / Studenten bedingter Selektionsbias.

Universitäten, die keine präzisen Angaben zur curricularen Implementierung der vier Erkrankungen machten, führten dies in den meisten Fällen auf das Fehlen eines strukturierten Lernzielkataloges zurück. Es erscheint daher wenig plausibel, dass die Lehrsituation an diesen Fakultäten grundlegend besser ist, als an den befragten Universitäten.

Ferner muss diskutiert werden, in wie weit die zum Zeitpunkt der Befragung abwesenden Studenten, zu einem Selektionsbias beigetragen haben. Auch hier erscheint es unwahrscheinlich, dass gerade diese Studenten über bessere Kenntnisse und Beratungsfähigkeiten als die befragte Stichprobe verfügten.

In einer Studie aus dem Jahr 1994 wurde die Kursanwesenheit von 197 Studenten in einem achtwöchigen Gynäkologiepraktikum mit ihren Noten in der Abschlussprüfung verglichen. Hier zeigte sich, dass Studenten, die mehr als 30\% der Zeit fehlten, signifikant schlechtere Prüfungsergebnisse erzielten als Studenten mit regelmäßiger Kursteilnahme (Riggs und Blanco 1994). Ähnliche Ergebnisse lieferte eine Untersuchung von 115 Medizinstudenten des ersten Studienjahres an der Universität von Washington. Während des Kurses wurden in regelmäßigen Abständen freiwillige Selbstevaluationen durchgeführt. Die Teilnahme an vier bis fünf der Selbstevalutionen und damit bestehende regelmäßige Kursteilnahme war mit einer Erfolgsrate von 83,3\% in der Abschlussprüfung korreliert, während eine Teilnahme an drei oder weniger als drei der Selbstevaluationen nur mit einer Erfolgsrate von 52,9\% verbunden war (Millis et al. 2009).

Dass der Großteil der Studenten die eigene Kompetenz, Raucher zu beraten, für gering hält, wurde bereits in einigen Studien gezeigt (Cantor et al. 1993, Raupach et al. 2009). Durch das Fehlen von Vergleichsvariablen konnte jedoch nicht genau beurteilt werden, ob die Studenten ihre Fähigkeiten generell unterschätzten oder ob es sich um spezifische, die Raucherberatung betreffende, Schwächen handelte. Dieser Problematik wurde entgegengewirkt, indem Selbsteinschätzungen zu anderen Erkrankungen in den Fragebogen mit aufgenommen wurden. Die Validität studentischer Selbsteinschätzungen wurde aufgrund einer hohen intra-individuellen Variabilität entsprechender Größen in Frage gestellt (Ward et al. 2002). In zwei kürzlich veröffentlichten Studien konnte jedoch gezeigt 
werden, dass die geforderten Selbstangaben gut mit objektiven Prüfvariablen korrelieren (Frank et al. 2005, Brooks et al. 2007). Darüber hinaus bleibt festzuhalten, dass subjektiv wahrgenommene Kompetenz nur durch Selbstangaben gemessen werden kann (Pederson et al. 2006). Außerdem erscheint es unwahrscheinlich, dass ein Student rauchende und alkoholabhängige Patienten berät, wenn er sich nicht kompetent genug dafür hält.

Die Studie wurde als Querschnittsuntersuchung durchgeführt, so dass sich die Angaben der einzelnen Studienjahre auf verschiedene Kohorten beziehen. Eine wahre Veränderung des Wissensstandes kann aber nur durch ein logitudinales Studiendesign eruiert werden. Ungeachtet dessen wurde vor nicht allzu langer Zeit eine angemessen hohe Valdität für Daten einer Querschnittsuntersuchung im Vergleich mit einer Längsschnittuntersuchung nachgewiesen (Cape et al. 2006).

\subsection{Schlussfolgerung und Ausblick}

Rauchen und gefährlicher Alkoholkonsum müssen als chronische Erkrankungen mit zentraler gesundheitspolitischer Bedeutung anerkannt und in der Ausbildung entsprechend behandelt werden. Dies fordert auch das von der Bundesregierung im Jahr 2003 ratifizierte internationale Rahmenabkommen zur Tabakkontrolle der WHO. Unterzeichner verpflichten sich nach Artikel 12 und 14 zur ausreichenden Aufklärung über Natur und Gefahr der Tabakabhängigkeit sowie die Implementation von Schulungsprogrammen für Gesundheitsberufe (World Health Organization 2003). Sechs Jahre später fühlten sich allerdings die wenigsten Medizinstudenten dazu in der Lage, rauchende (und alkoholabhängige) Patienten zu beraten.

Die Gesamtheit der Studienergebnisse legt nahe, dass zur Verbesserung der Lehrsituation ein Perspektivenwechsel von assoziierten Folgeschäden hin zu Prävention, Beratung und Entwöhnung erfolgen sollte. Medizinische Curricula müssen dahingehend umstrukturiert werden, dass Studenten nach Abschluss des Studiums fähig sind, Patienten und Patientinnen konsequent auf das Thema Rauchen und Rauchstopp und Alkoholkonsum sowie Alkoholentwöhnung anzusprechen. Um dieses Ziel zu erreichen, müssen anstelle von Vorlesungen mehr praxisbetonte Lehrmethoden eingeführt werden. Kleingruppenarbeit, Rollenspiele und Simulationspatienten erwiesen sich als effektive Lernformate für diesen Zweck und sollten schon früh im Studium beginnen (Leong et al. 2008, Shellenberger et al. 2009). Da summative Prüfungen einen besonders starken Lernanreiz darstellen (Raupach et al. 2010), sollten die unterrichteten Fähigkeiten darüber 
hinaus (Raucherberatung, Beratung alkoholabhängiger Patienten) auch Prüfungsinhalt werden (Geller et al. 2005). 


\section{Zusammenfassung}

Rauchen und übermäßiger Alkoholkonsum zählen weltweit $\mathrm{zu}$ den wichtigsten Risikofaktoren für vorzeitige Morbidität und Mortalität (Mathers und Loncar 2006), können aber effektiv von ärztlicher Seite behandelt werden (Fiore et al. 2008, Kaner et al. 2009). Eine leitliniengerechte Behandlung setzt jedoch eine adäquate medizinische Ausbildung voraus. Studien aus dem Ausland konnten curriculare Defizite und studentische Wissenslücken auf diesem Gebiet nachweisen; eine groß angelegte Untersuchung deutscher Hochschulen und Studenten lag bislang nicht vor.

Mittels Fragebogen wurden im Sommersemester 2009 erstmalig Daten zur curricularen Verankerung und zum studentischen Wissensstand in Bezug auf Krankheitsaspekte der Tabak- und Alkoholabhängigkeit erhoben und mit denen der arteriellen Hypertonie und des Diabetes mellitus verglichen. Es wurde dabei hypothetisiert, dass Abhängigkeitserkrankungen nicht mit derselben Intensität wie „klassische internistische“ Erkrankungen unterrichtet werden.

Daten zur Curriuclumanalyse konnten von insgesamt 25 Fakultäten erhoben werden. Dabei zeigte sich, dass Diabetes mellitus und arterielle Hypertonie mit durchschnittlich 315 beziehungsweise 275 Minuten deutlich umfangreicher unterrichtet wurden als Tabakund Alkoholabhängigkeit (135 und 180 Minuten). In den meisten Fällen wurden Vorlesungen als Lehrformat eingesetzt.

An der Studierendenbefragung beteiligten sich insgesamt 19.528 Studenten von 27 Fakultäten. Gegen Ende des Studiums kannten die Studenten zwar die Folgeerkrankungen von arterieller Hypertonie, Diabetes mellitus, Tabak- und Alkoholabhängigkeit, wie die Tabak- und Alkoholabhängigkeit jedoch behandelt werden, wusste weniger als die Hälfte aller Studenten. Effektive medikamentöse und verhaltenstherapeutische Entwöhnungsmaßnahmen wurden von der Mehrheit der Studenten unterschätzt. Nur jeder zwanzigste berichtete über praktischen Unterricht zur Tabakentwöhnung, so dass sich insgesamt auch nur sehr wenig Studenten für fähig hielten, eine adäquate Raucherberatung durchzuführen. Die Mehrheit der Studenten sprach sich für eine Intensivierung der universitären Lehre zu den Themen „Tabak- und Alkoholabhängigkeit" aus.

Im Einklang mit bereits publizierten Daten wird deutlich, dass Studenten durch ihre medizinische Ausbildung nicht genügend mit notwendigem Wissen zu Therapiestrategien in der Behandlung rauchender und alkoholabhängiger Patienten ausgestattet werden. Um diese Defizite zu beheben, müssen sich medizinische Curricula stärker auf Behandlungsoptionen, als auf Komplikationen der Erkrankungen konzentrieren und vermehrt praxisorientierte Lehrmethoden einsetzen. 


\section{Literaturverzeichnis}

Adler Al, Stevens RJ, Manley SE, Bilous RW, Cull CA, Holman RR, on behalf of the UKPDS Group (2003):

Development and progression of nephropathy in type 2 diabetes: The United Kingdom Prospective Diabetes Study (UKPDS 64). Kidney Int 63, 225-232

Alati R, Mamun AA, Williams GM, O'Callaghan M, Najman JM, Bor W (2006):

In utero alcohol exposure and prediction of alcohol disorders in early adulthood. Arch Gen Psychiatry $\underline{63}, 1009-1016$

Alzamora MT, Forés R, Baena-Díez JM, Pera G, Toran P, Sorribes $M$, Vicheto $M$, Reina MD, Sancho A, Albaladejo C, Llussà J; PERART/ARTPER study group (2010): The peripheral arterial disease study (PERART/ARTPER): prevalence and risk factors in the general population. BMC Public Health $\underline{10}, 38$

Anderson P (2006):

Global use of alcohol, drugs and tobacco. Drug Alcohol Rev 25 (6), 489-502

Anton RF, O'Malley SS, Ciraulo DA, Cisler RA, Couper D, Donovan DM, Gastfriend DR, Hosking JD, Johnson BA, LoCastro JS et al. (2006):

Combined pharmacotherapies and behavioral interventions for alcohol dependence: the COMBINE study: a randomized controlled trial. JAMA 295, 2003-2017

Aring AM, Jones DE, Falko JM (2005):

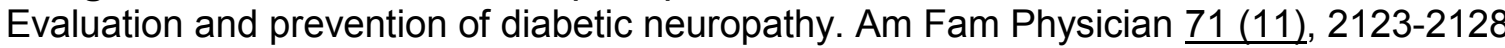

Aronson D (2008):

Hyperglycemia and the pathobiology of diabetic complications. Adv Cardiol 45, 1-16

Aveyard P, West R (2007):

Managing smoking cessation. BMJ $\underline{335}, 37-41$

Babor TF, Higgins-Biddle JC, Dauser D, Burleson JA, Zarkin GA, Bray J (2006):

Brief interventions for at-risk drinking: Patient outcomes and cost-effectiveness in managed care organizations. Alcohol \& Alcohol 41 (6), 624-631

Beks PJ, Mmackaay AJ, de Neeling JN, de Vries H, Bouter LM, Heine RJ (1995):

Peripheral arterial disease in relation to glycaemic level in an elderly Caucasian population: the Hoorn study. Diabetologica 38 (1), 86-96

Berglund M (2005):

A better widged? Three lessons for improving addiction treatment from a meta-analytical study. Addiction $\underline{100}, 742-750$

Bergmann E, Horch K:

Beiträge zur Gesundheitsberichterstattung des Bundes. Kosten alkoholassoziierter Krankheiten. Schätzungen für Deutschland. Hrsg.: Robert-Koch-Institut, Berlin 2002

Bondyl S, Victor J, Diemert L (2009):

Origin and use of the 100 cigarette criterion in tobacco surveys. Tob Control 18 , 317-323

Bottlender M( 2007):

One drink, one drunk - Ist kontrolliertes Trinken möglich? Psychotherapie, Psychosomatik, Med Psychol 57 (1), 32-38 
Brancati FL, Whelton PK, Randall BL, Neaton JD, Stamler J, Klag MJ (1997):

Risk of end-stage renal disease in diabetes mellitus: a prospective cohort study of men screened for MRFIT. Multiple Risk Factor Intervention Trial. JAMA 278 (23), 2069-2074

\section{Brenner H, Scharrer S (1996):}

Smoking habits of future physicians: a survey among medical students of a south German university. Soz Praventivmed 41,150-157

\section{Brewer C (2005):}

Supervised disulfiram is more effective in alcoholism than naltrexone or acamprosate - or even psychotherapy: how it works and why it matters. Adicciones 17 (4), 1-12

Brewster JM, Single, E, Ashley MJ, Chow YC, Skinner HA, Rankin JG (1990):

Preventing alcohol problems: survey of Canadian medical schools. CMAJ $\underline{143}, 1076-1082$

\section{Britton A, Marmot M (2004):}

Different measures of alcohol consumption and risk of coronary heart disease and allcause mortality: 11 year follow-up of the Whitehall II Cohort Study. Addiction 99,109-116

Britton J, Jarvis M, McNeill A, Bates C, Cuthbertson L, Godfrey C (2001):

Treating nicotine addiction: not a medical problem? Am J Respir Crit Care Med 164, 13-15

Brooks KR, Siegel B, Lash TL, Geller AC, Fitzgerald A, Tracy C, Prout MN (2007):

Validating medical students' self-report of smoking-related communication skills and edu-

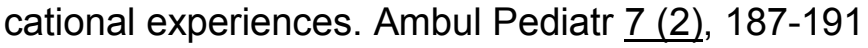

Buchanan TA, Kitzmiller JL (1994):

Metabolic interactions of diabetes and pregnancy. Annu Rev Med 4도, 245-260

Cahill K, Stead LF, Lancaster T (2008):

Nicotine receptor partial agonists for smoking cessation (Review). Cochrane Database of Systematic Reviews (3), CD006103

Cantor JC, Baker LC, Hughes RG (1993):

Preparedness for practice. Young physicians' views of their professional education. JAMA $\underline{270}, 1035-1040$

Cape G, Hannah A, Sellman D (2006):

A longitudinal evaluation of medical student knowledge, skills and attitudes to alcohol and drugs. Addiction 101, 841-849

\section{Centers for Disease Control and Prevention:}

The Health Consequences of Smoking: A Report of the Surgeon General. U.S. Department of Health and Human Services, National Center for Chronic Disease Prevention and Health Promotion, Office on Smoking and Health: Atlanta 2004

Centers for Disease Control and Prevention (2006):

Use of cigarettes and other tobacco products among students aged 13-15 yearsworldwide, 1999-2005. MMWR 55 (20), 553-556

Chatkin J, Chatkin G (2009):

Learning about smoking during medical school: are we still missing opportunities? Int $\mathrm{J}$ Tuberc Lung Dis $\underline{13}$, 429-437 
Clarke R, Emberson J, Fletcher A, Breeze E, Marmot M, Shipley MJ (2009):

Life expectancy in relation to cardiovascular risk factors: 38 year follow-up of 19000 men in the Whitehall study. BMJ $\underline{339}$, b3513

Coder B, Meyer C, Freyer-Adam J, John U:

Alkohol - Zahlen und Fakten zum Konsum; in: Jahrbuch Sucht 2009. Deutsche Hauptstelle für Suchtfragen (Hrsg.). Geesthacht, Neuland 2009: 21-50

Conroy MB, Majchrzak NE, Regan S, Silverman CB, Schneider LI, Rigotti NA (2005):

The association between patient-reported receipt of tobacco intervention at a primary care

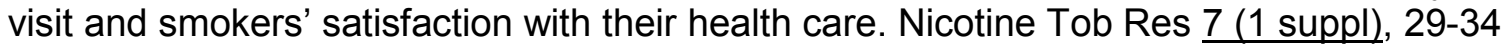

Corrao G, Bagnardi V, Zambon A, La Vecchia C (2004):

A meta-analysis of alcohol consumption and the risk of 15 diseases. Preventive Medicine $\underline{38}, 613-619$

Cummings SR, Stein MJ, Hansen B, Richard RJ, Gerbert B, Coates TJ (1989):

Smoking counseling and preventive medicine. A survey of internists in private practices and a health maintenance organization. Arch Intern Med 149 (2), 345-349

Deutsche Gesellschaft für Hypertonie und Prävention:

Behandlung der arteriellen Hypertonie (S2-Leitlinie). Hrsg.: Deutsche Hochdruckliga e.V., Heidelberg 2008

Doll R, Peto R, Boreham J, Sutherland I (2004):

Mortality in relation to smoking: 50 years' observations on male British doctors. BMJ $\underline{328}$, 1519-1527

\section{Esson M, Leeder S:}

The Millenium Development goals and tobacco control: an opportunity for global partnership. Hrsg.: World Health Organization, Genf 2004

Ezzati M, Lopez A, Rodgers A, Vander Hoorn S, Murray C, Group CR (2002):

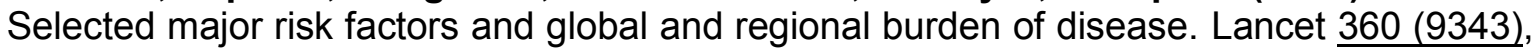
$1347-1360$

Farrington J (2004):

Noncommunicable diseases in the WHO European Region: the challenge. Fact Sheet EURO 06/04. Copenhagen, World Health Organization. Heruntergeladen von http://www.euro.who.int/mediacentre/PR/2004/2004090_1

Fatjó F, Sancho-Bru P, Fernández-Solà J, Sacanella E, Estruch R, Bataller R, Nicolàs JM (2007):

Up-regulation of myocardial L-type Ca2+ channel in chronic alcoholic subjects without cardiomyopathy. Alcohol Clin Exp Res $\underline{31}$, 1099-1105

Ferketich AK, Khan Y, Wewers ME (2006):

Are physicians asking about tobacco use and assisting with cessation? Results from the 2001-2004 national ambulatory medical care survey (NAMCS). Prev Med $\underline{43}$, 472-476

Ferri M, Amato L, Davoli M (2006):

Alcoholics Anonymous and other 12-step programmes for alcohol dependence. Cochrane Database of Systematic Reviews (3), CD005032 
Ferry LH, Grissino LM, Runfola PS (1999):

Tobacco dependence curricula in US undergraduate medical education. JAMA 282, 825829

Fiore MC (2000):

Treating tobacco use and dependence: an introduction to the US Public Health Service Clinical Practice Guideline. Respir Care 45 (10), 1196-1199

Fiore MC, Jaén CR, Baker TB, Bailey WC, Benowitz NL, Curry SJ, Dorfman SF, Froelicher ES, Goldsstein MG, Healton CG et al.:

Treating Tobacco Use and Dependence: 2008 Update. Clinical Practice Guideline. 2008, U.S. Department of Health and Human Services. Public Health Service.: Rockville, 2008

Fisher JC, Mason RL, Keeley KA, Fisher JV (1975):

Physicians and alcoholics. The effect of medical training on attitudes toward alcoholics. $J$ Stud Alcohol $\underline{36}, 949-955$

Fleming M, Barry K, Davis A, Kropp S, Kahn R, Rivo M (1994):

Medical education about substance abuse: changes in curriculum and faculty between 1976 and 1992. Academic Medecine 69, 362-369

Fleming MF (2004/2005):

Screening and Brief Intervention in primary care settings. Alcohol Research and Health $\underline{28}$ (2), 57-62

Frank E, McLendon L, Denniston M, Fitzmaurice D, Hertzberg V, Elon L (2005):

Medical students' self-reported typical counseling practices are similar to those assessed with standardized patients. Med Gen Med 7 (1), 2

Frydrych LM, Greene BJ, Blondell RD, Purdy CH (2009):

Self-help program components and linkage to aftercare following inpatient detoxification. $J$ Addict Dis 28 (1), 21-27

Fuller JH, Elford J, Goldblatt P, Adelstein AM (1983):

Diabetes mortality: new light on an underestimated public health problem. Diabetologia $\underline{24}, 336-334$

Gärtner B, Meyer C, Freyer-Adam J, John U:

Alkohol - Zahlen und Fakten zum Konsum; in: Deutsche Hauptstelle für Suchtfragen (Hrsg.) Jahrbuch Sucht 2010. Geesthacht: Neuland 2010, 21-47

Garbutt JC (2010):

Efficacy and Tolerability of Naltrexone in the Management of Alcohol Dependence. Curr Pharm Design 16, 2091-2097

Geller AC, Zapka J, Brooks KR, Dube C, Powers CA, Rigotti N, O'Donnell J, Ockene J (2005):

Tobacco control competencies for US medical students. Am J Public Health $\underline{95}, 950-955$

George JT, Warriner DA, Anthony J, Rozario KS, Xavier S, Jude EB, McKay GA (2008):

Training tomorrow's doctors in diabetes: self-reported confidence levels, practice and perceived training needs of post-graduate trainee doctors in the UK. A multi-centre survey. BMC Med Educ 8 (22), 1 - 7 
Glass IB (1988):

Substance abuse and professional education: a tops-down or bottoms-up approach? $\mathrm{Br} \mathrm{J}$ Addict $\underline{83}, 999-100$

GTSS Collaborative Group (2006):

Tobacco use and cessation counselling: Global Health Professionals Survey Pilot Study, 10 countries, 2005. Tob Control 15 (2), ii31-34

Guindon GE, Boisclair D (2003):

Past, current and future trends in tobacco use. Washington, DC, World Bank (http://www.worldbank.org/tobacco/pdf/ Guindon- Past,20 current-20whole.pdf, accessed 5 December 2007)

Hanefeld M, Fischer S, Julius U, Schulze J, Schwanebeck U, Schmechel H, et al. (1996):

Risk factors for myocardial infarction and death in newly detected NIDDM: the Diabetes Intervention Study, 11-year follow-up. Diabetologia 39 (12), 1577-1583

Hanke M, John U (2003):

Tabak- oder alkohol-attributable stationäre Behandlungen. Dtsch Med Wochenschr $\underline{128}$, $1387-1390$

Hauner H (2005):

Epidemiologie und Kostenaspekte des Diabetes mellitus in Deutschland. Dtsch Med Wochenschr 130, 64-65

Heller G (2004):

Wie häufig sind Diabetes-bedingte Amputationen unterer Extremitäten in Deutschland. Dtsch Med Wochenschr 129, 429-433

Hoch E, Muehlig S, Hoefler M Lieb R, Wittchen HU (2004):

How prevalent is smoking and nicotine dependence in primary care in Germany? Addiction $\underline{99}, 1586-1598$

Hughes JR (1999):

Four beliefs that may impede progress in the treatment of smoking. Tob Control $\underline{8(3)}$, 323-326

Hughes JR, Keely J, Naud S (2004):

Shape of the relapse curve and long-term abstinence among untreated smokers. Addiction 99 (1), 29-38

Hughes JR, Stead LF, Lancaster T (2007):

Antidepressants for smoking cessation. Cochrane Database of Systematic Reviews (1), CD000031

Humair JP, Cornuz J (2003):

A new curriculum using active learning methods and standardized patients to train residents in smoking cessation. J Gen Intern Med 18 (12), 1023-1027

Icks A, Trautner C, Haastert B, Berger M, Giani G (1997):

Blindness due to diabetes: population-based age and sex.specific incidence rates. Diabet Med 14 (7), 571-575

International Federation of Diabetes:

The Diabetes Atlas (Bd. 3). International Diabetes Federation (Hrsg.), Brussels 2006 
Jaen CR, Stange KC, Nutting PA (1994):

Competing demands of primary care: A model for the delivery of clinical preventive services. Journal of Family Practice 38, 166-171

Jahnsen K, Strube H, Starker A:

Hypertonie. Gesundheitsberichterstattung des Bundes. Heft 43. Hrsg.: Robert-KochInstitut, Berlin 2008

Jennings GL (1997):

Exercise and blood pressure: Walk, run or swim? J Hypertens $\underline{15}, 567-569$

John U, Hanke M (2002):

Alcohol-attributable mortality in a high per capita consumption country - Germany. Alcohol \& Alcoholism $\underline{37(6)}, 581-585$

John U, Hanke M (2003):

Tobacco- and alcohol-attributable mortality and years of potential life lost in Germany. Eur J Public Health $\underline{13}$, 275-277

John U, Hapke U, Rumpf HJ, Hill A, Dilling H:

Prävalenz und Sekundärprävention von Alkoholmissbrauch und -abhängigkeit in der medizinischen Versorgung. Nomos, Baden-Baden 1996

Kahan M, Midmer D, Wilson L, Borsoi D (2006):

Medical students' knowledge about alcohol and drug problems: results of the medical council of Canada examination. Subst Abus $\underline{27}, 1-7$

Kaner EF, Dickinson HO, Beyer FR, Campbell F, Schlesinger C, Heather N, Saunders JB, Burnand B, Pienaar ED:

Effectiveness of brief alcohol interventions in primary care populations (Review). The Cochrane Library (2).The Cochrane Collaboration. Published by JohnWiley \& Sons, Ltd, 2009

Kearney PM, Whelton M, Reynolds K, Muntner P, Whelton PK, He J (2005):

Global burden of hypertension: analysis of worldwide data. Lancet 365 (9455), 217-223

Klatsky AL (2009):

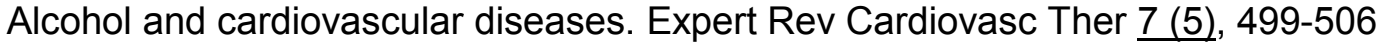

Kongsved S, Basnov M, Holm-Christensen K, Hjollund N (2007):

Reponse rate and completeness of questionnaires: a randomized study of internet versus paper-and-pencil versions. J Med Internet Res $\underline{9(3)}$, e25

Konnopka A, König HH (2007):

Direct and indirect costs attributable to alcohol consumption in Germany. Pharmacoeconomics $\underline{25}(7), 605-618$

Kosowicz LY, Pfeiffer CA, Vargas M (2007):

Long-term retention of smoking cessation counseling skills learned in the first year of medical school. J Gen Intern Med 22, 1161-1165

Köster I, von Ferber L, Ihle P, Schubert I, Hauner H (2006):

The cost burden of diabetes mellitus: the evidence from Germany- the CoDiM Study. Diabetologica $\underline{49}, 1498-1504$ 
Krampe H, Ehrenreich H (2010):

Supervised Disulfiram as Adjunct to Psychotherapy in Alcoholism Treatment. Curr Pharm Design 16, 2076-2090

Krampe H, Stawicki S, Hoehe MR, Ehrenreich H (2007):

Outpatient Long-term Intensive Therapy for Alcoholics (OLITA): a successful biopsychosocial approach to the treatment of alcoholism. Dialogues Clin Neurosci $\underline{9}, 399-$ 412

Kraus L, Bauernfeind R (1998):

Repräsentativerhebungen zum Gebrauch psychoaktiver

Substanzen bei Erwachsenen in Deutschland 1997. Sucht 44, Sonderheft 1

Kraus L, Rösner S, Baumeister SE, Pabst A, Steiner S (2008):

Epidemiologischer Suchtsurvey 2006. Repräsentativerhebung zum Gebrauch und Missbrauch psychoaktiver Substanzen bei Jugendlichen und Erwachsenen in Berlin, IFTBerichte 176, 15-16. München: IFT Institut für Therapieforschung

Lancaster T, Stead LF (2005):

Self-help interventions for smoking cessation. Cochrane Database of Systematic Reviews (3), CD001118

\section{Lancaster T, Stead L, Silagy C, Sowden A (2000):}

Regular review: Effectiveness of interventions to help people stop smoking: findings from the Cochrane Library. BMJ $\underline{321}, 355-358$

\section{Lasswell AB, Liepman MR, McQuade WH, Wolfson MA, Levy SM (1993):}

Comparison of primary care residents' confidence and clinical behavior in treating hypertension versus treating alcoholism. Acad Med $\underline{68}, 580-582$

Lehnert H, Wittchen H, Pittrow D, Bramlage P, Kirch W, Böhler S, Höfler M, Ritz E (2005):

Prävalenz und Pharmakotherapie des Diabetes mellitus in der primärärztlichen Versorgung. Dtsch Med Wochenschr 130, 323-328

Lemmer B, Middeke M, Schaaf B, Eckes, L (2008):

Prescribing practices and morning blood pressure control: results of a large-scale, primary-care study conducted in Germany. J Hum Hypertens $\underline{22}$, 295-297

Leong SL, Lewis PR, Curry WJ, Gingrich DL (2008):

Tobacco world: evaluation of a tobacco cessation training program for third-year medical students. Acad Med $\underline{83}$, 25-28

Levenkron JC, Greenland P, Bowley N (1990):

Teaching risk-factor counseling skills: a comparison of two instructional methods. Am J Prev Med $\underline{6}, 29-34$

Lokke A, Lange $P$, Scharling $H$, Fabricius $P$, Vestbo J (2006):

Developing COPD: a 25 year follow up study of the general population. Thorax $\underline{61}, 935-$ 939

Lopez A, Mathers C, Ezzati M, Jamison D, Murray CJ:

Measuring the Global Burden of Disease and Risk Factors, 1990-2001; in: Global Burden of Disease and Risk Factors ed. (1-13). Oxford University Press. New York 2006 
Löwel H, Stieber J, König W, Thorand B, Hörmann A, Gostomzyk J, Keil U (1999):

Das Diabetes-bedingte Herzinfarktrisiko in einer süddeutschen Bevölkerung: Ergebnisse der MONICA-Augsburg Studien 1985 - 1994. Diabetes Stoffwechsel $\underline{8}, 11-21$

Löwel H, Meisinger C, Heier M, Hymer H, Alte D, Völzke H (2006):

Epidemiology of hypertension in Germany. Selected results of population-representative cross-sectional studies. Dtsch Med Wochenschr 131, 2586-2591

Lu L, Liu Y, Zhu W, Shi J, Liu Y, Ling W, Kosten TR (2009):

Traditional medicine in the treatment of drug addiction. Am J Drug Alcohol Abuse 35 (1), $1-11$

Mann K (2002):

Neue ärztliche Aufgaben bei Alkoholproblemen. Dtsch Ärztebl 99, A632-A644

Mann K, Kapp B (1995):

Zur Lehre in Suchtmedizin - eine Befragung von Studenten und Professoren; in: Mann K, Buchkremer G (Hrsg.) Suchtforschung und Suchttherapie in Deutschland. Neuland Hamm. Sucht, Sonderheft, 38-40

Mann K, Lehert P, Morgan MY (2004):

The Efficacy of Acamprosate in the Maintenance of Abstinence in Alcohol-Dependent Individuals: Results of a Meta-Analysis. Alcohol Clin Exp Res 28 (1), 51-63

Marcea IT, Kuhlmann T, Summa-Lehmann P, Reymann G, Reinert T, Endres J, Lütz M, Gilles A, Elsner H, Plath J et al. (2001):

Leitlinien für die Erbringung medizinischer Leistungen in der Diagnostik, Behandlung und Rehabilitation von Patienten mit Abhängigkeit und / oder schädlichem Gebrauch von psychotropen Substanzen für das Fachgebiet Psychiatrie/Psychotherapie. Suchttherapie $\underline{2}, 45-50$

Martin S, Dreyer M, Kiess W, Lüdecke HJ, Müller UA, Schatz H, WaldhäusI W:

Evidenzbasierte Leitlinie der DDG - Therapie des Diabetes mellitus Typ 1. Hrsg.: Scherbaum WA, Kerner W, Deutsche Gesellschaft für Diabetes, 2007

Mathers C, Loncar D (2006):

Projections of global mortality and burden of disease from 2002 to 2030 . PLoS Med $\underline{3}$, e442

Matthaei S, Bierwirth R, Fritsche A, Gallwitz B, Häring HU, Joost HG, Kellerer M, Kloos C, Kunt T, Nauck M et al.:

Medikamentöse antihyperglykämische Therapie des Diabetes mellitus Typ 2; in: Update der Evidenzbasierten Leitlinie der Deutschen Diabetes-Gesellschaft. Hrsg. Scherbaum WA, Haak T, 2008

McCord EC, Smorowski-Garcia K, Doughty A (1997):

Assessment at one school of students' abilities and confidence in diabetic patients' education. Acad Med $\underline{72}, 1116-1118$

Midmer D, Kahan M, Wilson L (2008):

Medical students' experiences with addicted patients: a web-based survey. Subst Abus $\underline{29}$ (1), 25-32

Miller NS, Sheppard LM, Colenda CC, Magen J (2001):

Why physicians are unprepared to treat patients who have alcohol- and drug-related disorders. Acad Med $\underline{76}, 410-418$ 


\section{Miller WR, Sanchez VC:}

Motivating young adults for treatment and lifestyle change, in: G. Howard (Ed.), Issues in alcohol use and misuse by young adults. University of Notre Dame Press, Notre Dame Indiana 1994, 55-82

Miller WR, Wilbourne PL (2002):

Mesa Grande: a methodological analysis of clinical trials of treatments for alcohol use disorders. Addiction 97, 265-277

Millis RM, Dyson S, Cannon D (2009):

Association of classroom participation and examination performance in a first-year medical school course. Adv Physiol Educ $\underline{33}, 139-$ 143

Mons U, Pötschke-Langer M:

Rauchfreie Gaststätten in Deutschland 2009: Steigende Zustimmung in der Bevölkerung. Hrsg.: Deutsches Krebsforschungszentrum, Heidelberg 2009

Moon RY, Horne RS, Hauck FR (2007):

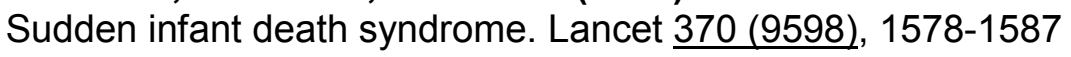

Moos RH, Finney JW, Ouimette PC, Suchinsky RT (1999):

A comparative evaluation of substance abuse treatment: I. Treatment orientation, amount of care, and 1-year outcomes. Alcohol Clin Exp Res 23, 529-536

Moos R, Schaefer J, Andrassy J, Moos B (2001):

Outpatient mental health care, self-help groups, and patients' 1-year treatment outcomes. J Clin Psychol $\underline{57}, 1-1$

Moyer A, Finney JW (2002):

Outcomes for untreated individuals involved in randomized trials of alcohol treatment. $J$ Subst Abuse Treat 23 (3), 247-252

Müller-Riemenschneider F, Nocon M, Willich S (2010):

Prevalence of modifiable cardiovascular risk factors in German adolescents. European Journal of Cardiovascular Prevention and Rehabilitation $\underline{00}, 1-7$

Mumtaz S, Ashfaq T, Siddiqui H (2009):

Knowledge of medical students regarding diabetes mellitus at Ziauddin University, Karachi. J Pak Med Assoc 59 (3), 163-166

Neubauer S, Welte R, Beiche A, Koenig H-H, Buesch K, Leidl R (2006):

Mortality, morbidity and costs attributable to smoking in Germany: update and a 10-year comparison. Tob Control 15, 464-471

Obialo Cl, Hewan-Lowe K, Fulong B (2002):

Nephrotic proteinuria as a result of essential hypertension. Kidney Blood Press Res $\underline{25}$ (4), 250-254

Oscar-Berman M, Marinković K (2007):

Alcohol: effects on neurobehavioral functions and the brain. Neuropsychol Rev 17 (3), 239-257

Ott P, Benke I, Stelzer J, Köhler C, Hanefeld M (2009):

Diabetes in Germany (DIG) study. A prospective 4-year-follow-up study on the quality of treatment for type 2 diabetes in daily practice].Dtsch Med Wochenschr 134 (7), 291-297 
Paterson NE (2009):

Behavioural and pharmacological mechanisms of bupropion's anti-smoking effects: recent preclinical and clinical insights. Eur J Pharmacol $\underline{603(1-3)}, 1-11$

Pederson LL, Blumenthal DS, Dever A, McGrady G (2006):

A web-based smoking cessation and prevention curriculum for medical students: why, how, what, and what next. Drug Alcohol Rev 25, 39-47

Pine D, Sullivan S, Conn SA, David C (1999):

Promoting tobacco cessation in primary care practice. Primary Care $\underline{26}, 591-610$

Pitel AL, Beaunieux H, Witkowski T, Vabret F, Guillery-Girard B, Quinette P, Desgranges B, Eustache F (2007):

Genuine episodic memory deficits and executive dysfunctions in alcoholic subjects early in abstinence. Alcohol Clin Exp Res 31, 1169-1178

Polednak AP (2005):

Recent trends in incidence rates for selected alcohol-related cancers in the United States. Alcohol Alcohol $\underline{40}, 234-238$

Proude EM, Conigrave KM, Britton A, Haber PS (2008):

Improving alcohol and tobacco history taking by junior medical officers. Alcohol Alcohol $\underline{43}, 320-325$

Prugger C, Heuschmann P, Keil U (2006):

Epidemiology of hypertension in Germany and worldwide. Herz 31, 287-293

Puddey IB, Beilin LJ (2006):

Alcohol is bad for blood pressure. Clin Exp Pharmacol Physiol 33 (9), 847-852

Puddey IB, Beilin LJ, Rakie V (1997):

Alcohol, hypertension and the cardiovascular system: a critical appraisal. Addiction Biol $\underline{2}, 159-170$

Puska P, Mendis S, Porter D (2003):

Chronic disease - key risk factors include high cholesterol, high blood pressure, low fruit and vegetable intake. WHO global strategy on diet, physical activity and health, http://www.who.int/dietphysicalactivity/publications/facts/riskfactors/en/index.html

Ramstedt M (2004):

Alcohol and pancreatitis mortality in the population level: experiences from 14 western countries. Addiction $\underline{99}$, 1255-1261

Rathmann W, Strassburger K, Heier M, Holle R, Thorand B, Giani G, Meisinger C (2009):

Incidence of type 2 diabetes in the elderly German population and the effect of clinical and lifestyle risk factors: KORA S4/F4 cohort study. Diabet Med 26, 1212-1219

\section{Raupach T, Shahab L, Baetzing S, Hoffmann B, Hasenfuss G, West R, Andreas S (2009):}

Medical students lack basic knowledge about smoking: Findings from two European medical schools. Nictotine Tob Res $\underline{11}, 1-7$

\section{Raupach T, Hanneforth N, Anders S, Pukrop T, Cate OT, Harendza S (2010):}

Impact of teaching and assessment format on electrocardiogram interpretation skills. Med Educ 44 (7), 731-740 
Raupach T, Merker J, Hasenfuss G, Andreas S, Pipe A (2011):

Knowledge gaps about smoking cessation in hospitalized patients and their doctors. Eur $\mathrm{J}$ Cardiovasc Prev Rehabil 18 (2), 334-341

\section{Rehm J, Mathers C, Popova S, Thavorncharoensap M, Teerawattananon Y, Patra J (2009):}

Global burden of disease and injury and economic cost attributable to alcohol use and alcohol-use disorders. Lancet $\underline{373}, 2223-2233$

Richmond RL, Kehoe L (1997):

Smoking behaviour and attitudes among Australian medical students. Med Educ 31, 169176

Richmond RL, Debono DS, Larcos D, Keho L (1998):

Worldwide survey of education on tobacco in medical schools. Tob Control $\underline{7}, 247-252$

Richmond R, Zwar N, Taylor R, Hunnisett J, Hyslop F (2009):

Teaching about tobacco in medical schools: a worldwide study. Drug Alcohol Rev 28 (5), 484-449

Riggs JW, Blanco JD (1994):

Is there a relation between student lecture attendance and

clinical science subject examination score? Obstet Gynecol 84, 311-313

Ritson EB (1991):

Undergraduate alcohol education. Alcohol Alcohol 26, 273-275

Roche AM (1992):

Drug and alcohol medical education: evaluation of a national programme. $\mathrm{Br} \mathrm{J}$ Addict $\underline{87}$, 1041-1048

Roche AM, Eccleston P, Sanson-Fisher R (1996):

Teaching smoking cessation skills to senior medical students: a block-randomized controlled trial of four different approaches. Prev Med 25, 251-258

Roddy E, Rubin P, Britton J (2004):

A study of smoking and smoking cessation on the curricula of UK medical schools. Tob Control $13,74-77$

Rodriguez BL, D'Agostino R, Abbott RD, Kagan A, Burchfield CM, Yano K, Ross GW, Silbershatz H, Higgins MW, Popper J et al. (2002):

Risk of hospitalized stroke in men enrolled in the Honolulu Heart Program and the Framingham Study: a comparison of incidence and risk factor effects. Stroke $\underline{33}, 230-236$

Rösner S, Hackl-Herrwerth A, Leucht S, Vecchi S, Srisurapanont M, Soyka M (2010): Opioid antagonists for alcohol dependence. Cochrane Database of Systematic Reviews 12, CD001867

Rogers JM (2009):

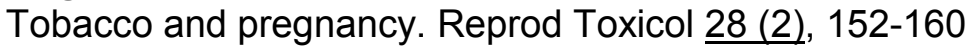

Roglic G, Unwin N (2010):

Mortality attributable to diabetes: Estimates for the year 2010. Diabetes Res Clin Pract $\underline{87}$ (1), $15-19$ 
Rumpf HJ, Meyer C (2000):

Inanspruchnahme suchtspezifischer Hilfen von Alkoholabhängigen und -missbrauchern: Ergebnisse der TACOS Bevölkerungsstudie. Sucht 46, 9-17

Sacks FM, Svetkey LP, Vollmer WM, Appel LJ, Bray GA, Harsha D, Obarzanek E, Conlin PR, Miller ER 3rd, Simons-Morton DG et al. (2001):

Effects on blood pressure of reduced dietary sodium and the Dietary Approaches to Stop Hypertension (DASH) diet. DASH Sodium Collaborative Research Group. N Engl J Med $\underline{344}, 3-10$

Sanchez-Craig M, Davila R, Cooper G (1996):

A self-help approach for high-risk drinking: effect of an initial assessment. J Consult Clin Psychol $\underline{64}$ (4), 694-700

Schneider H, Lischinski M, Jutzi E (1993):

Survival of diabetic patients at 30 -year follow- up with reference to a closed population. Z Arztl Fortbild (Jena) 87 (4), 323-327

Schubert HD (1998):

Ocular manifestations of systemic hypertension. Curr Opin Ophthalmol $\underline{9(6)}, 69-72$

Schuckit MA (2009):

Alcohol use disorders. Lancet $\underline{373}, 492-501$

Shafey O, Dolwick S, Guindon GE:

Tobacco Control Country Profiles 2003, American Cancer Society, Atlanta Georgia 2003

Sharma AM, Wittchen HU, Kirch W, Pittrow D, Ritz E, Goke B, Lehnert H, Tschope D, Krause P, Hofler, M et al.; HYDRA Study Group (2004):

High prevalence and poor control of hypertension in primary care: cross-sectional study. $J$ Hypertens $\underline{22}$, 479-486

Shellenberger S, Seale JP, Harris DL, Johnson JA, Dodrill CL, Velasquez MM (2009): Applying team-based learning in primary care residency programs to increase patient alcohol screenings and brief interventions. Acad Med 84, 340-346

Simpson CR, Hannaford PC, Ritchie LD, Sheikh A, Williams D (2011):

Impact of the pay-for-performance contract and the management of hypertension in Scottish primary care: a 6-year population-based repeated cross-sectional study. $\mathrm{Br} \mathrm{J}$ Gen Pract $\underline{61 \text { (588) }}, 443-451$

Skoeries BA, Ulbricht S, Koepsell S, Rumpf HJ, John U, Meyer C (2010):

Readiness to provide smoking cessation counselling--results of a survey among general practitioners in Brandenburg. Gesundheitswesen $\underline{72}$ (4), 228-232

Smith DR, Leggat PA (2007):

An international review of tobacco smoking among medical students. J Postgrad Med $\underline{53}$, $55-62$

Spangler JG, George G, Foley KL, Crandall SJ (2002):

Tobacco intervention training: current efforts and gaps in US medical schools. JAMA $\underline{288}$, 1102-1109 
Spittell PC, Spittell JA Jr, Joyce JW, Tajik AJ, Edwards WD, Schaff HV, Stanson AW (1993):

Clinical features and differential diagnosis of aortic dissection: experience with 236 cases (1980 through 1990). Mayo Clin Proc $\underline{68}$, 642-651

Springer CM, Tannert Niang KM, Matte TD, Miller N, Bassett MT, Frieden TR (2008): Do medical students know enough about smoking to help their future patients? Assessment of New York City fourth-year medical students' knowledge of tobacco cessation and treatment for nicotine addiction. Acad Med $\underline{83}$, 982-989

Statistisches Bundesamt Deutschland:

Ergebnisse der Todesursachenstatistik für Deutschland - Ausführliche vierstellige ICD10Klassifikation 2009. Heruntergeladen von https://wwwec.destatis.de/csp/shop/sfg/bpm.html.cms.cBroker.cls?Cmspath

$=$ struktur, vollanzeige.csp\&ID=1026368 am 21.02.2011

Statistisches Bundesamt Wiesbaden:

Gesundheitsberichterstattung des Bundes (1998). Gesundheitsbericht für Deutschland, Statistisches Bundesamt Wiesbaden, 1999

Statistisches Bundesamt Wiesbaden:

Mikrozensus - Fragen zur Gesundheit. Rauchgewohnheiten der Bevölkerung, Statistisches Bundesamt Wiesbaden, 2011

Stead LF, Bergson G, Lancaster T (2008a):

Physician advice for smoking cessation. Cochrane Database Syst Rev 2, CD000165

Stead LF, Perera R, Bullen C, Mant D, Lancaster T (2008b):

Nicotine replacement therapy for smoking cessation. Cochrane Database of Systematic Reviews 1, Art. No.: CD000146. DOI: 10.1002/14651858.CD000146.pub3

Strandberg A, Strandberg T, Pitkälä K, Salomaa V, Tilvis R, Miettinen T (2008):

The effect of smoking in midlife on health-related quality of life in old age: a 26-year prospective study. Arch Intern Med 168, 1968-1974

Straus S, Majumdar S, McAlister F (2002):

New Evidence for Stroke Prevention: scientific review. JAMA 288, 1388-1395

Szucs S, Sarvary A, McKee M, Adany R (2005):

Could the high level of cirrhosis in central and eastern Europe be due partly to the quality of alcohol consumed? An exploratory investigation. Addiction 100, 536-542

Teo KK, Ounpuu S, Hawken S, Pandey MR, Valentin V, Hunt D, Diaz R, Rashed W, Freeman $R$, Jiang $L$, Zhang $X$, Yusuf $S$ on behalf of the INTERHEART Study Investigators (2006):

Tobacco use and risk of myocardial infarction in 52 countries in the INTERHEART study: a case-control study. Lancet $\underline{368}, 647-658$

Tessier JF, Freour P, Crofton J, Kombou L (1989):

Smoking habits and attitudes of medical students towards smoking and antismoking campaigns in fourteen European countries. Eur J Epidemiol $\underline{5}$, 311-321

Tessier JF, Fréour PP, Nejjari C (1992a):

Smoking behaviour and attitudes of medical students towards smoking and anti-smoking campaigns: a survey in 10 African and Middle Eastern countries. Tob Control 1, 95-101 
Tessier JF, Freour P, Belougne D, Crofton J (1992b):

Smoking habits and attitudes of medical students towards smoking and antismoking campaigns in nine Asian countries. The Tobacco and Health Committee of the International Union Against Tuberculosis and Lung Diseases. Int J Epidemiol 21, 298-304

Tessier JF, Fréour PP, Nejjari C (1993):

Smoking behaviour and attitudes towards smoking of medical students in Australia, Japan, USA, Russia, and Estonia. Tob Control 2, 24-29

Teyssen S, Singer MV (2003):

Alcohol-related diseases of the oesophagus and stomach. Best Pract Res Clin Gastroenterol $17(4), 557-573$

Thamm M (1999):

Blutdruck in Deutschland - Zustandsbeschreibung und Trends. Gesundheitswesen $\underline{61}$ (Sonderheft 2), 90-93

Thefeld W (1999):

Bundesgesundheitssurvey: Prävalenz des Diabetes mellitus in der erwachsenen Bevölkerung. Deutschlands Gesundheitswesen 61 (Sonderheft 2), 85-89

Thomson AD, Marshall EJ (2006):

The natural history and pathophysiology of Wernicke's encephalopathy and Korsakoff 's psychosis. Alcohol Alcohol $\underline{41}, 151-158$

Thun MJ, Peto R, Lopez AD, Monaco JH, Hanley SJ, Heath CW, Doll R (1997):

Alcohol consumption and mortality among middle-aged and elderly US Adults. N Eng J Med $\underline{337(24)}, 1705-1714$

Twardella D, Brenner H (2005):

Lack of training as a central barrier to the promotion of smoking cessation: a survey among general practitioners in Germany. Eur J Public Health 15 (2), 140-145

Upchurch GR Jr, Schaub TA (2006):

Abdominal aortic aneurysm. Am Fam Physician 73 (7), 1198-1204

Uyarel H, Ozdol C, Gencer AM, Okmen E, Cam N (2005):

Acute alcohol intake and QT dispersion in healthy subjects. J Stud Alcohol $\underline{66}, 555-58$

Vallejo M, Jordan C, Diaz M, Comeche M, Ortega J (2007):

Psychological assessment via the internet: a reliability and validity study of online (vs. paper and pencil) versions of the General Health Questionnaire-28 (GHQ-28) and the Symptoms-Check-List-90-Revised (SCL-90-R). J Med Internet Res 9 (1), e2

Vasilaki EI, Hosier SG, Cox WM (2006):

The efficacy of motivational interviewing as a brief intervention for excessive drinking: a

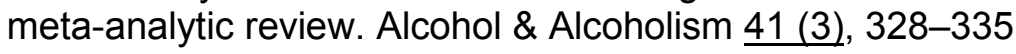

Vinik Al, Maser RE, Mitchell BD, Freeman R (2003):

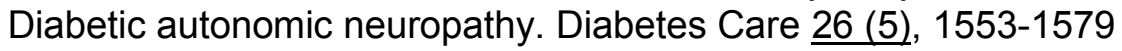

Vittadini G, Buonocore M, Colli G, Terzi M, Fonte R, Biscaldi G (2001):

Alcoholic polyneuropathy: a clinical and epidemiological study. Alcohol Alcohol $\underline{36}(5)$, 393-400 


\section{Vogt F, Hall S, Marteau TM (2006):}

General practitioners' beliefs about effectiveness and intentions to prescribe smoking cessation medications: Qualitative and quantitative studies. BMC Public Health $\underline{6}, 277$

von Troschke J, Westenhoff F (1989):

Einstellungen von Medizinstudenten in der Bundesrepublik Deutschland zum Rauchen. Soz Praeventivmed 34 (3), 119-123

Ward M, Gruppen L, Regehr G (2002):

Measuring self-assessment: current state of the art.

Adv Health Sci Educ Theory Pract $\underline{7}, 63-80$

Warren CW, Jones NR, Chauvin J, Peruga A; GTSS Collaborative Group (2008):

Tobacco use and cessation counselling: cross-country. Data from the Global Health Professions Student Survey (GHPSS), 2005-7. Tob Control 17 (4), 238-247

West R, McNeill A, Raw M (2000):

Smoking cessation guidelines for health professionals: an update. Health Education Authority. Thorax $\underline{55(12)}$, 987-999

Whelton PK, Appel LJ, Espeland MA, Applegate WB, Ettinger WH Jr, Kostis JB, Kumanyika S, Lacy CR, Johnson KC, Folmar S, Cutler JA (1998):

Sodium reduction and weight loss in the treatment of hypertension in older persons: a randomized controlled trial of nonpharmacologic interventions in the elderly (TONE). TONE Collaborative Research Group. JAMA 279, 839-846

White AR, Rampes H, Liu JP, Stead LF, Campbell J (2011):

Acupuncture and related interventions for smoking cessation. Cochrane Database of Systematic Reviews 1, CD000009

Wienhold:

Die vergessene Mehrheit; in: Sucht Heft 1, Bonn 2000

Wild S, Roglic C, Green A (2003):

Global prevalence of diabetes: estimates for the year 2000 and projections for 2030. Diabetes Care 27, 1047-1053

Williams GC, Levesque C, Zeldman A, Wright S, Deci EL (2003):

Health care practitioners' motivation for tobacco-dependence counseling. Health Educ $\operatorname{Res} \underline{18}, 538-553$

Willigendael EM, Teijink JA, Bartelink ML, Kuiken BW, Boiten J, Moll FL, Büller HR, Prins MH (2004):

Influence of smoking on incidence and prevalence of peripheral arterial disease. $\mathrm{J}$ Vasc Surg $\underline{40(6)}, 1158-1165$

Wolf PA, D'Agostino RB, Belanger AJ, Kannel WB (1991):

Probability of stroke: a risk profile from the Framingham Study. Stroke $\underline{22}, 312-318$

Wolf-Maier K, Cooper R, Banegas J, Giampoli S, Hense H, Joffres M, et al. (2003):

Hypertension prevalence and blood pressure levels in 6 European countries, Canada, and the United States. JAMA 289, 2363-2369

Wong TY, Mitchell P (2007):

The eye in hypertension. Lancet 369 (9559), 425-435 
World Health Organization:

WHO Framework Convention on Tobacco Control. Hrsg. World Health Organization, Genf 2003

World Health Organization:

The European health report 2005 : public health action for healthier children and populations. Hrsg.: World Health Organization, Europa 2005

World Health Organization:

Highlights on health in Germany 2004. Hrsg.: Wolrd Health Organization. Heruntergeladen von http://www.euro.who.int/highlights, 2006

Yusuf S, Hawken S, Ounpuu S, Dans T, Avezum A, Lanas F, McQueen M, Budaj A, Pais P, Varigos J et al. (2004):

Effect of potentially modifiable risk factors associated with myocardial infarction in 52 countries (the INTERHEART study): case-control study. Lancet $\underline{364}$, 937-952 


\section{Danksagung}

Mein besonderer Dank gilt Herrn Dr. Tobias Raupach für die Möglichkeit unter seiner Leitung zu promovieren. Ohne inn wäre diese Studie nicht realisierbar gewesen! Vielen Dank für die Vielzahl an konstruktiven Gesprächen, die ständige Bereitschaft, meine Fragen ausführlich zu beantworten, und die hervorragende Betreuung während des gesamten Projekts.

Ganz herzlich bedanken möchte ich mich bei Herrn Prof. G. Burckhardt, für die Übernahme der Schirmherrschaft der Studie und für die unterstützende Kraft bei der Kontaktierung der Studienzentren.

Ebenfalls sehr herzlich bedanken möchte ich mich bei Herrn Dr. N. Schneider und Herrn Dipl-Päd. H. Krampe, die mit viel konstruktiver Kritik an der Gestaltung des Fragebogens und des Studientreffens mitgewirkt haben. Herrn Krampe möchte ich darüber hinaus danken, dass ich mit Fragen zur aktuellen Studienlage zum Thema Alkoholabhängigkeit immer zu ihm kommen konnte und er mir diese stets ausführlich und hilfreich beantwortet hat.

Bei Herrn Prof. E. Brunner und Herrn Prof. T. Beissbarth möchte ich mich für die Hilfe bei der statistischen Auswertung und für das mir so oft fehlende fachmännische „Know-how“ bedanken.

Zuletzt möchte ich mich bei Herrn C. Münscher bedanken, ohne dessen geduldige Art noch bis heute keine 20.000 Fragebögen gescannt und verarbeitet worden wären. 


\section{Lebenslauf}

Am 18. August 1984 wurde ich als zweites Kind von Herrn Dr. Hubert Rainer Müllner und Frau Mechthild Elfriede Barbara Müllner, geb. Rathscheck, in Königstein im Taunus geboren. Von 1990 bis 1994 besuchte ich die Albert - von - Reinach Grundschule in Fischbach am Taunus. Nach der Grundschulzeit erfolgte ein Wechsel auf die weiterführende „Staufenschule“ im selben Ort, die ich nach einem Jahr jedoch wieder verließ und mich für das Gymnasium der St. Angela Schule in Königstein im Taunus entschied. Dort verbrachte ich die Mittel- bis Oberstufe und schloss meine Schulzeit im Juni 2003 mit der allgemeinen Hochschulreife (Durchschnittsnote 1,3) ab. Im April 2004 immatrikulierte ich mich an der Georg - August - Universität in Göttingen und bestand im März 2006 den 1. Abschnitt der Ärztlichen Prüfung (Durchschnittsnote 1,5). Von September 2007 bis Juli 2008 studierte ich als Erasmus-Stipendiatin an der „Université Victor Segalen“ in Bordeaux. Um experimentelles Arbeiten kennenzulernen, absolvierte ich unter Leitung von Herrn Prof. Dr. L. Walter ein studienbegleitendes Laborpraktikum in der Abteilung Primatengenetik im Deutschen Primatenzentrum in Göttingen. Von Juli 2007 bis September 2007 und August 2008 bis Dezember 2008 beschäftigte ich mich mit der Charakterisierung der Expression von MIC-Genen im Testis. Während meiner klinischen Studienzeit war ich zudem mehrfach als unterstützende Kraft für die Lehre in den Abteilungen Hämatologie und Onkologie, sowie Kardiologie und Pneumologie tätig. Im Dezember des Jahres 2008 begann ich meine Doktorarbeit unter der Leitung von Herrn PD Dr. T. Raupach in der Abteilung Kardiologie und Pneumologie der Universitätsmedizin Göttingen. Jeweils eine Hälfte des chirurgischen Tertials meines praktischen Jahres verbrachte ich am Singapore General Hospital in Singapur und am Hôpital Pellegrin in Bordeaux. Den ersten Teil meines internistischen Tertials absolvierte ich ebenfalls in Bordeaux, auf einer hämatologischonkologischen Station im Hôpital St- André. Für den zweiten Teil wählte ich einen gastroenterologischen Schwerpunkt und ging in die Ammerland Klinik in Westerstede. Mein Wahltertial belegte ich am Institut für Humangenetik der Universitätsmedizin Göttingen. Im November 2010 schloss ich das Studium der Humanmedizin mit dem 2. Abschnitt der Ärztlichen Prüfung ab (Durchschnittsnote 1,5).

Mittlerweile arbeite ich als Assistenzärztin in der Abteilung Innere Medizin, Schwerpunkt Gastroenterologie im DRK-Westend, Berlin. 